\title{
A!
}

This is an electronic reprint of the original article.

This reprint may differ from the original in pagination and typographic detail.

Autere, Anton; Jussila, Henri; Dai, Yunyun; Wang, Yadong; Lipsanen, Harri; Sun, Zhipei

\section{Nonlinear Optics with 2D Layered Materials}

Published in:

Advanced Materials

DOI:

10.1002/adma.201705963

Published: 01/01/2018

Document Version

Peer reviewed version

Please cite the original version:

Autere, A., Jussila, H., Dai, Y., Wang, Y., Lipsanen, H., \& Sun, Z. (2018). Nonlinear Optics with 2D Layered Materials. Advanced Materials, 30(24), [1705963]. https://doi.org/10.1002/adma.201705963

This material is protected by copyright and other intellectual property rights, and duplication or sale of all or part of any of the repository collections is not permitted, except that material may be duplicated by you for your research use or educational purposes in electronic or print form. You must obtain permission for any other use. Electronic or print copies may not be offered, whether for sale or otherwise to anyone who is not an authorised user. 
DOI: 10.1002/ (adma.201705963)

Article type: Review

\title{
Nonlinear Optics Based on Two-dimensional Layered Materials
}

Anton Autere $^{\S}$, Henri Jussila ${ }^{\S}$, Yunyun Dai ${ }^{\S}$, Yadong Wang, Harri Lipsanen and Zhipei Sun*

A. Autere, Dr. H. Jussila, Dr. Y. Dai, Y. Wang, Prof. H. Lipsanen, and Prof. Z. Sun

Department of Electronics and Nanoengineering, Aalto University, Tietotie 3, FI-02150,

Finland

E-mail: Zhipei.sun@aalto.fi

${ }^{\S}$ A.A, Y.D and H.J contributed equally to this work.

Keywords: Nonlinear optics, 2D materials, Harmonic generation, Four-wave mixing, Ultrafast lasers, Quantum photonics, Attosecond physics, THz technology, Frequency comb

\begin{abstract}
Two-dimensional layered materials (2DLMs) have been a subject of intense research for a wide variety of applications (e.g., electronics, photonics and optoelectronics), due to their unique physical properties. Most recently, increasing research efforts on 2DLMs have been projected towards the nonlinear optical properties of 2DLMs, which are not only fascinating from the fundamental science point-of-view but also intriguing for various potential applications. Here, we review the current state of the art in the field of nonlinear optics based on 2DLMs and their hybrid structures (e.g., mixed-dimensional heterostructures, plasmonic structures, and silicon/fibre integrated structures). We also present several potential perspectives and possible future research directions of these promising nanomaterials for nonlinear optics.
\end{abstract}

\section{Introduction}

Nonlinear optics is the branch of optics which examines the light behaviour in nonlinear media. ${ }^{[1,2]}$ Although the nonlinear optical (NLO) responses of materials are typically inherently weak $^{[1,2]}$, the NLO materials play an increasingly important role in all aspects of photonics (i.e., photon generation, manipulation, transmission, detection and imaging ${ }^{[1-3,4]}$ ). Indeed, these NLO materials have enabled various currently widely-used photonic devices (e.g., pulsed lasers $^{[5]}$, optical switches ${ }^{[6]}$, optical modulators ${ }^{[7]}$, photodetectors ${ }^{[8]}$, and optical memories ${ }^{[9]}$ ), underscoring the unparalleled advantages of optical techniques over their electronic counterparts. Some examples of NLO devices include but are not limited to nonlinear saturable absorbers for ultrafast pulsed lasers ${ }^{[5]}$ which are widely used in medicine and industrial material 
processing applications, soliton devices for large-scale telecommunication networks ${ }^{[4]}$, and frequency converters ${ }^{[10]}$ for bio-imaging technologies. ${ }^{[11]}$ Current NLO devices are normally based on bulk crystals (e.g., silica optical fiber, beta barium borate (BBO), lithium niobate $\left.\left(\mathrm{LiNbO}_{3}\right)\right)$ or traditional semiconductor materials that are combined with various optical confinement methods (e.g., tight-focusing, photonic crystal structures ${ }^{[12,13]}$ ). Today, we are envisaging a large range of exciting nonlinear nanophotonic applications (including on-chip nanophotonics ${ }^{[14]}$, quantum nanophotonics ${ }^{[15]}$, nonlinear plasmonics ${ }^{[16]}$ ). These applications have been advanced by a plethora of remarkable concept demonstrations mainly using traditional NLO materials. However, the current solutions of conventional nonlinear optics have hit a technical limit imposed by the traditional materials (e.g., relatively low NLO susceptibility ${ }^{[1,2,4]}$ ) and their available fabrication and integration technologies ${ }^{[1,2,4]}$, which cannot fully satisfy the current growing needs. For example, the integration of NLO materials with photonic integrated circuits (PICs) is highly desired for many applications, ranging from on-chip light sources for silicon photonics ${ }^{[17]}$ to emerging integrated quantum technologies. ${ }^{[18]}$ However, there are substantial challenges in integrating traditional NLO materials with other material platforms for on-chip applications. Therefore, it is significantly important for future photonics and optoelectronics to discover new materials with large NLO responses that can be chip-integrated to tackle the upcoming challenges of nanophotonics. These new materials are anticipated to provide innovative approaches to enable novel devices with new functionalities, high performance and new device features (e.g., reduced complexity, size and cost).

Two-dimensional (2D) layered materials (2DLMs) are atomically thin crystals which are currently at the centre of significant research effort. ${ }^{[19]}$ The intensive research efforts on 2DLMs were initially ignited by the ground-breaking work on graphene. ${ }^{[20]}$ Since then, the interest research towards graphene and other 2DLMs has been focusing not only on their unique electrical properties, but also on their fascinating optical, mechanical, thermal, and chemical properties. ${ }^{[19-21,22]}$ Indeed, these 2DLMs have exhibited many remarkable optical properties (e.g., ultrafast broadband optical response ${ }^{[23-27,28]}$, large optical nonlinearities ${ }^{[19,29-32]}$, and strong excitonic effects ${ }^{[33]}$ ), which have already enabled diverse new conceptual photonic devices fundamentally different from those based on traditional bulk materials. ${ }^{[19,31,33-35,36]}$ In this review paper, we present recent advances in 2DLMs based nonlinear optics, their applications and future perspectives. In Section 2, we discuss the fundamentals of different NLO processes and the general characteristics of 2DLMs, which make them promising 
candidates for NLO applications. This is then followed by a survey of the current state of the art of 2DLMs based nonlinear optics. At the end, we conclude with application perspectives on 2DLMs based nonlinear optics in Section 4.

\section{Fundamentals of 2DLMs based nonlinear optics}

\subsection{Fundamentals of nonlinear optics}

The optical response of a material to an applied optical field can be expressed by expanding the polarization $\mathbf{P}(t)$, as a power series in terms of the optical field strength $\mathbf{E}(t)$, as follows: ${ }^{[1,2]}$

$$
P(t)=\chi^{(1)} E(t)+\chi^{(2)} E(t)^{2}+\chi^{(3)} E(t)^{3}+\cdots,
$$

where $\chi^{(n)}$ is the $n^{\text {th }}$-order NLO susceptibility. The first term $\chi^{(1)}$ describes the conventional linear optical effects, such as refraction and absorption. However, if the optical field is intense enough or the NLO susceptibility is large, the higher-order $(n \geq 2)$ terms become significant ${ }^{[1,2]}$, which can give rise to radiation at frequencies (e.g., sum frequencies of the input light signals) different from the input light frequency. The second-order NLO effects, including second-harmonic generation (SHG, Figure 1(a)), sum- and difference frequency generation (SFG, DFG, Figure 1(a)), optical rectification and the Pockels effect, are described by the second-order NLO susceptibility $\chi^{(2)}$ in Equation 1 . The third-order NLO effects, such as thirdharmonic generation (THG), four-wave mixing (FWM) and intensity dependent refractive index change (optical Kerr effect and saturable absorption (SA)), arise from the third-order NLO susceptibility $\chi^{(3)}$ in the equation. High-order multiphoton absorption/scattering/ luminescence, and high harmonic generation (HHG), on the other hand, are described by the $\mathrm{n}^{\text {th }}$-order NLO susceptibility $\chi^{(\mathrm{n})}$, in which $n$ is typically larger than 5 . The interaction strength of the nonlinear processes ${ }^{[1,2]}$ typically decreases with $n$. Therefore, the second- and third-order nonlinear effects are the most commonly observed processes and are thus widely used for various applications (e.g., imaging ${ }^{[11]}$, frequency conversion ${ }^{[1,2,37]}$, ultrafast lasers $^{[5]}$, modulators $^{[7]}$, Figure 1(b)).

NLO processes are commonly explained with photon diagrams (Figure 1(a)). In general, harmonic generation is utilized to convert an optical signal to higher frequencies. In SHG (THG), two (three) incident photons at the frequency $\omega$ create light radiation at the frequency $2 \omega(3 \omega)$, as shown in Figure 1(a). As discussed, SHG and THG are the most frequently used NLO processes for various frequency conversion applications. Note that NLO processes (in particular various parametric processes, such as SHG, SFG, FWM) are typically phase sensitive, therefore efficient nonlinear frequency conversion requires a proper phase relationship between 
the interacting waves (i.e., phase-matching ${ }^{[1,2]}$ ). To achieve ideal phase-matching, the common methods are the use of crystal birefringence and the periodic poling of the crystal. ${ }^{[1,2]}$ In DFG, the frequency of the generated photons is the difference of the two input frequencies, (i.e., $\left.\omega_{3}=\omega_{1}-\omega_{2}\right)$, shown in Figure 1(a). When two optical waves at frequencies $\omega_{1}$ and $\omega_{2}$ interact in a $\chi^{(2)}$ nonlinear crystal, the input light at frequency $\omega_{2}$ can be amplified in the DFG process. This is called optical parametric amplification (OPA), which can create tuneable light amplifiers by mixing the output of two lasers at different frequencies. When such a amplification process happens inside an optical resonator, the gain from OPA can produce coherent light oscillation if it is larger than the cavity loss. ${ }^{[1,2]}$ These kinds of devices are called optical parametric oscillators (OPO) and commonly used to produce broad wavelengthtuneable coherent light sources (e.g., from the near- to mid-infrared ${ }^{[38]}$ or even terahertz $(\mathrm{THz})^{[1,2]}$ spectral regions) which are difficult to achieve with conventional lasers.

Because the input optical field and the dielectric polarization in a material are vector quantities, the NLO susceptibility is a tensor, which depends on the structure of the material. For example, $\chi^{(2)}$ is a third-rank tensor with 27 Cartesian components. A complete description of the NLO interaction in the case of SFG, therefore, requires 12 of these tensors yielding a total of 324 different and complex components that need to be specified.$^{[1,2]}$ Fortunately, intrinsic permutation symmetry and crystal symmetry decrease the number of independent components. Due to the higher tensor rank of the $\chi^{(2)}$ and $\chi^{(3)}$, the NLO responses of a material can be anisotropic, even if the linear optical response is isotropic. ${ }^{[39]}$ This makes NLO effects (e.g., SHG DFG, THG, FWM, Figure 1(a)) a very sensitive method for probing the crystal orientation of materials. ${ }^{[1,2]}$ The second-order and other even-order NLO effects can only occur in a material that lacks the centre of inversion symmetry. ${ }^{[1,2]}$ Therefore, the second-order NLO effects, such as SHG, are dipole-forbidden in centrosymmetric materials. However, secondorder NLO effects can occur at the surface of a centrosymmetric material, due to broken inversion symmetry at the interface of two different materials.${ }^{[40]}$ On the contrary, third-order NLO effects are allowed in all materials regardless of the crystal symmetry. It is worth noting that Raman scattering is also a NLO process. It is a very powerful method for characterization of materials (including 2DLMs), which will not be discussed here due to space limitations and a few recently-published review articles. ${ }^{[41]}$ Nowadays, nonlinear optics is a key enabling technology for a large range of applications, including light generation (e.g., ultrafast pulse 
generation, optical parametric generation (OPG), $\mathrm{THz}$ generation), quantum photonics, attoscience, imaging and sensing, as shown in Figure 1(b).

\subsection{Fundamentals of optical properties of 2DLMs}

\subsubsection{Graphene}

Graphene has numerous remarkable physical properties (e.g., ultrahigh carrier mobility, ultrafast broadband optical response, high flexibility, robustness and environmental stability). ${ }^{[19-21,22]}$ These intriguing properties arise from its unique crystal structure in which each carbon atom is covalently bonded to three other carbon atoms forming a hexagonal lattice. Since the conduction and valence bands of graphene meet at the $\mathbf{K}$ point of the Brillouin zone, graphene is semimetal, see Figure 2(a). The energy-momentum relation of charge carriers is linear near the Dirac point, and therefore electrons and holes inside graphene behave as massless Dirac fermions ${ }^{[20,21,22]}$, which gives rise to the unique optical properties of graphene. ${ }^{[19,26]}$ For instance, monolayer graphene has broadband absorption of $\sim 2.3 \%$ (defined by the fine-structure constant ${ }^{[42]}$ ). This makes graphene a promising material for broadband optical devices (e.g., photodetectors ${ }^{[19,26]}$, saturable absorbers ${ }^{[30,43,44]}$, and modulators ${ }^{[31]}$ ), covering an extremely wide spectral range from the ultraviolet to microwave regions. The NLO properties of graphene (e.g., $\mathrm{SA}^{[29,30]}$, $\mathrm{THG}^{[45-48]}$, $\mathrm{FWM}^{[49-52]}$, self-phase modulation ${ }^{[53]}$, $\mathrm{HHG}^{[54]}$, coherent optical injection ${ }^{[55]}$, optical limiting ${ }^{[56]}$ and nonlinear Kerr effect ${ }^{[57,58]}$ ) have recently gathered huge interest. The typical ultrafast (e.g., down to few hundred femtoseconds ${ }^{[59,60]}$ ) carrier dynamics enables ultrafast NLO responses of graphene. Due to the centrosymmetric crystal structure of graphene, the second-order NLO effects are not allowed in graphene. However, SHG has been observed from the interface in different hybrid graphene devices regardless of the inversion symmetry in graphene. ${ }^{[61,62]}$ Furthermore, the inversion symmetry can be broken by physical (e.g., structural engineering ${ }^{[63,64]}$, external electric field ${ }^{[65]}$ and multi-layer structures ${ }^{[65-67]}$ ) or chemical methods (e.g., chemical doping or etching ${ }^{[68]}$ ). Very recently, electrically tuneable THG and FWM in graphene have also been demonstrated. ${ }^{[69,70]}$ The results show that the ability to tune the linear and nonlinear responses of graphene ${ }^{[48,67,71,72,73,74]}$ can potentially enable various electrically-tuneable NLO devices based on graphene for future photonics and optoelectronics (e.g., harmonic generation, frequency comb, THz generation). 


\section{WILEY-VCH}

\subsubsection{Transition metal dichalcogenides}

Transition metal dichalcogenides (TMDs) are the most studied member of the 2D material family beyond graphene. ${ }^{[19,31,33-35,36]}$ TMDs are semiconductor materials with the formula of $\mathrm{MX}_{2}$, where $\mathrm{M}$ refers to transition metal element (e.g., Mo, W, Re) and X to chalcogen element (e.g., S, Se, or Te). In monolayer TMD crystals, one transition metal atom is sandwiched between two layers of chalcogen atoms. Weak van der Waals forces bound each $\mathrm{MX}_{2}$ monolayer together in few-layer and bulk TMDs. These TMDs normally possess a layerdependent bandgap. For example, $\mathrm{MoS}_{2}, \mathrm{MoSe}_{2}, \mathrm{WS}_{2}$ and $\mathrm{WSe}_{2}$ are indirect bandgap semiconductors in bulk form, but at single atomic layer their bandgap is direct. ${ }^{[36]}$ Note that bulk TMDs with direct bandgap (e.g., $\operatorname{ReS}_{2}{ }^{[19,75]}$ ) also exists. As monolayer TMDs are typically semiconductor materials, their optical properties differ from that of graphene significantly, which can be utilized in various applications. For example, the direct bandgap in monolayer TMDs enables atomically-thin light emitting diodes. ${ }^{[76]}$ The bandgap of the most studied TMDs (e.g., $\mathrm{MoS}_{2}, \mathrm{MoSe}_{2}, \mathrm{MoTe}_{2}, \mathrm{WS}_{2}, \mathrm{WSe}_{2}, \mathrm{WTe}_{2}$ ) is between $\sim 0.7$ and $1.9 \mathrm{eV} .^{[19,34,36,77]}$ Monolayer TMD materials can even absorb $20 \%$ of the light at a specific resonance energy [19,34,36] , and the photoluminescence (PL) behaviour of TMDs is dominated by excitons. ${ }^{[78]}$ Furthermore, trion lines have also been observed in their PL spectra. ${ }^{[79]}$

NLO properties of TMD materials are quite fascinating. Thus far, for instance, $\mathrm{SA}^{[80,81]}$, $\mathrm{SHG}^{[82-92]}, \mathrm{SFG}^{[93]}, \mathrm{THG}^{[94-99]}$, fourth-harmonic generation $(\mathrm{FHG})^{[95]}$, optical limiting ${ }^{[100]}$, $\mathrm{HHG}^{[101]}$ and $\mathrm{FWM}^{[93,102]}$ have been observed in monolayer and few-layer TMDs. In addition, since TMDs with odd number of layers have no inversion symmetry, second-order (e.g., SHG) and other even-order NLO effects are allowed in these TMD crystals which are not present in graphene or typical TMD crystals with even number of layers. ${ }^{[82]}$ Note that, 3R-phase TMD crystals have no inversion symmetry despite the parity of the layer number. Therefore the evenorder NLO responses (e.g., SHG ${ }^{[103,104]}$ ) are always permitted. Furthermore, the lack of inversion symmetry enables various spintronic and valleytronic applications. ${ }^{[105]}$ In fact, experimental evidence of valley physics has been seen in monolayer TMDs, where the carrier populations in distinct valleys (e.g., $\pm \mathbf{K}$ positions of the Brillouin zone as schematically depicted in Figure 2(b)) can be controlled by varying the polarization of the excitation light. [105] 


\section{WILEY-VCH}

\subsubsection{Black phosphorus}

Black phosphorus (BP) is a layered material consisting of phosphorus atoms. ${ }^{[106]}$ In contrast to graphene and TMDs, BP has its own unique optical properties. For example, its direct electronic bandgap depends on the crystal thickness and can be tuned from $\sim 0.3$ to $\sim 2 \mathrm{eV}$ (corresponding to the wavelength range from $\sim 4$ to $0.6 \mu \mathrm{m}) .{ }^{[107,108]}$ This is particularly appealing for mid-infrared photonics and optoelectronics, as the bandgap of BP can bridge the gap between the zero-bandgap graphene and the relatively large bandgap TMDs. ${ }^{[31,109]}$ Furthermore, the crystal structure of BP is anisotropic (see Figure 2(c)), leading to anisotropic optical ${ }^{[107,109-111]}$, electrical and thermal properties. ${ }^{[112]}$ As a result, BP is a birefringent and dichroic material, opening the field of 2DLMs based anisotropic optics. ${ }^{[110,111]}$ This is especially interesting for nanophotonic applications requiring the polarization control of the light. ${ }^{[110,111]}$ BP has high NLO susceptibility $\left(\sim 10^{-19} \mathrm{~m}^{2} / \mathrm{V}^{2}\right)\left(\right.$ e.g., $\mathrm{THG}^{[113-115]}, \mathrm{FWM}^{[116,117]}$ and $\left.\mathrm{SA}^{[111,118,119]}\right)$. However, the lack of sufficient stability can lead to rapid degradation of the electronic and optical properties of BP ${ }^{[120]}$, limiting the current potential of BP outside the proof-of-principle research demonstrations. Nevertheless, to improve the long-term stability of BP devices, some strategies (e.g., capping layer protection by using h-BN layers, ligand surface coordination $^{[121]}$ and polymers ${ }^{[119]}$, or directly with BP polymer ${ }^{[118]}$ ) have been demonstrated.

\subsubsection{Other $2 D L M S$}

In recent years, hundreds of 2DLMs have been studied with different properties (yet unexplored) opening the possibility of discovering novel physics for various applications. Thus far, these "new" 2DLMs can exhibit a rich variety of physical properties, ranging from that of a wideband insulator to a narrow-gap semiconductor, to a semimetal or metal. For example, hexagonal boron nitride (h-BN) is an extremely important member of the 2D material family, originally known from its insulator properties. In fact, $\mathrm{h}-\mathrm{BN}$ is at present commonly used as an atomically flat and ultra-clean substrate (and a tunnelling barrier as well) for 2D hybrid structures. ${ }^{[19]}$ For instance, the high electron mobility values of graphene are typically achieved when graphene is transferred on h-BN crystals. ${ }^{[122]}$ However, h-BN is not only interesting for electronics but also promising for photonics. In particular, the large band gap of h-BN suggests that h-BN crystals may be potentially used for ultraviolet light generation in the future nonlinear optics, which will be discussed in Section 4.7. At the moment, the observation that defects in h-BN crystal can be used for single photon generation ${ }^{[123,124]}$ (similarly as nitrogen vacancies in diamond ${ }^{[125]}$ ) has gathered large interest, deserving further investigation with NLO 
approaches. In addition, group III and IV metal chalcogenides have been experimentally demonstrated with high NLO responses. ${ }^{[92,99,126]}$ For example, various 2DLMs (e.g., GeSe and $\mathrm{SnS}^{[127]}$ ) have been predicted to have giant NLO responses (e.g., the susceptibility tensor element $\chi_{\text {xyx }}^{(2)}$ of $5.16 \times 10^{6} \mathrm{pm}^{2} / \mathrm{V}$ for monolayer $\left.\mathrm{GeSe}^{[127]}\right)$. Further, the extensive understanding of optical nonlinearity in graphene potentially suggests unique NLO responses of other massless Dirac fermions that can be observed in many other novel 2DLMs, such as topological insulators ${ }^{[128]}$, Dirac and Weyl semimetals ${ }^{[129]}$, and even superconductors.

\subsubsection{DLMs based hybrid structures}

Current research efforts on 2DLMs based hybrid structures are actively involved in the combination of 2DLMs with other low-dimensional materials, such as zero-dimensional (e.g., metallic plasmonic nanoparticles ${ }^{[130]}$, organic molecules, quantum dots, fullerene ${ }^{[131]}$, onedimensional (e.g., nanowires, nanotubes, quantum wires, nanoribbons), and three-dimensional bulk materials (e.g., inorganic and oxide semiconductors, metals $\left.{ }^{[132]}\right) .{ }^{[131]}$ The creation of the different mixed-dimensional hybrid structures has introduced a new paradigm with enormous potential for novel devices. ${ }^{[19,131]}$ For example, thanks to the recent significant advances in the fabrication methods, it has now become possible to stack different 2DLMs together and construct $2 \mathrm{D}$ material heterostructures $(2 \mathrm{DHs}) .{ }^{[68,133]}$ In this case, atomically thin 2D layers with different properties can be fabricated individually and then stacked together to construct van der Waals-bonded heterostructures, in which each layer can be designed independently to realize functions that were not previously available. ${ }^{[31,133]}$ There have been significant efforts to investigate different 2DHs, including but not limited to graphene-BN, graphene-BP, TMDBN, TMD-BP, TMD-graphene and TMD-TMD combinations. ${ }^{[31,133]}$ These mixed-dimensional heterostructures are particularly interesting for photonics and optoelectronics. ${ }^{[31,133]}$ For example, the realization of different heterojunctions (e.g., Type-I, Type-II, and Type-III) provides means to engineer the linear and NLO properties of 2DLMs for various desired functions that are not possible with individual 2DLMs. ${ }^{[134]}$ The formation of indirect excitons (i.e., exciton between two different TMDs) has been observed in 2DHs. ${ }^{[135]}$ As a result, 2DHs can be fully utilized to create novel devices for numerous applications (e.g., exciton-based optical nonlinearity tuning $\left.{ }^{[136]}\right)$. It has been shown that NLO processes in these mixeddimensional heterostructures are driven by a coherent superposition of the optical fields from individual layers $^{[103,137,138,139]}$, with an interference phase difference dependent on the stacking orientation of 2DLMs. Therefore, the NLO response of various heterostructures can be 
coherently enhanced if a constructive interference heterostructure superlattice is used. At the moment, these mixed-dimensional structures have been used for nonlinear optics (e.g., second harmonic generation ${ }^{[93]}, \mathrm{SA}^{[140]}$, but not fully explored yet.

\section{State-of-the-art of 2DLMs based nonlinear optics}

Research on 2DLMs based nonlinear optics has attracted huge interest, and this has led to a significant progress over the past decade. Table 1 summarizes typical NLO processes in 2DLMs and their key parameters (e.g., the NLO susceptibility, conversion efficiency). A large range of different 2DLMs (e.g., graphene, $\mathrm{MoS}_{2}$, InSe, h-BN, $\mathrm{ReS}_{2}, \mathrm{GaSe}, \mathrm{BP}$ ) have been fabricated with different methods (e.g., mechanical exfoliation (ME), Chemical vapor deposition (CVD), liquid-phase exfoliation (LPE), pulsed laser deposition (PLD)) for various NLO processes (e.g., SHG, THG, FWM). In this Section, the current state-of-the art of 2DLMs based representative NLO processes is presented.

\subsection{Harmonic generation}

After the first experimental demonstrations of harmonic generation in 2DLMs (e.g., surface SHG in graphene ${ }^{[61]}$, SHG in $\mathrm{MoS}_{2}{ }^{[82-84]}$, THG from graphene ${ }^{[46,47,95]}$ and $\mathrm{MoS}_{2}{ }^{[95,97]}$ ), significant research efforts have been directed to this topic. Since then, demonstrations of SHG and THG have been reported from a wide range of monolayer and few-layer 2DLMs, including $\mathrm{BP}^{[114,115,141]}, \mathrm{WSe}_{2}{ }^{[85,86,91]}, \mathrm{WS}_{2}{ }^{[85,87]}, \mathrm{MoSe}_{2}{ }^{[90,142]}, \mathrm{GaSe}^{[92,99,143]}, \mathrm{GaTe}^{[126]}, \mathrm{h}-\mathrm{BN}^{[82,144]}$, $\mathrm{MoTe}_{2}{ }^{[88,145]}, \mathrm{InSe}^{[146]}, \mathrm{ReS}_{2}{ }^{[98]}$; and $\mathrm{AgInP}_{2} \mathrm{~S}_{6}{ }^{[147]}$ Most of these results have revealed that 2DLMs exhibit exceptionally large NLO susceptibilities (e.g., $\left.\chi^{(2)} \approx \mathrm{nm} \mathrm{V}^{-1}\right)^{[82,84]}$, implying huge potential of these materials for various applications (e.g., ultrafast pulse characterization $^{[89]}$, telecom wavelength conversion ${ }^{[148,149]}$ ).

\subsubsection{Second harmonic generation}

The lack of inversion symmetry can give rise to strong SHG signal under intense optical pump. ${ }^{[82-84]}$ The observation of SHG in monolayer $\mathrm{MoS}_{2}$ with $\sim 800 \mathrm{~nm}$ excitation wavelength was reported in References ${ }^{\left[{ }^{[2-84]}\right.}$. It demonstrated that SHG in monolayer $\mathrm{MoS}_{2}$ is orders of magnitude larger than that of bilayer or bulk $\mathrm{MoS}_{2}$, as expected from their crystal symmetry properties. ${ }^{[82]}$ Note that SHG signal also has been observed in monolayer graphene ${ }^{[61,62]}$, evennumber layered $\mathrm{MoS}_{2}{ }^{[150]}$ and $\mathrm{WSe}_{2}{ }^{[151]}$ flakes, and spiral $\mathrm{WS}_{2}$ nanosheets ${ }^{[152]}$ by symmetry breaking. In addition to the NLO response at the wavelength of $\sim 800 \mathrm{~nm}$, another interesting wavelength range is $\sim 1550 \mathrm{~nm}$, commonly used for optical telecommunication related applications. With the fundamental wavelength close to $1550 \mathrm{~nm}$, the reported $\chi^{(2)}$ values (i.e., 
in the range of few $\mathrm{pmV}^{-1}$ for $\mathrm{MoS}_{2}{ }^{[95,96,153]}$ and $\mathrm{MoSe}_{2}{ }^{[90,142]}$ ) are clearly lower than that measured with $800 \mathrm{~nm}$ lasers. The difference between results measured at $800 \mathrm{~nm}$ and 1550 nm was attributed to resonant enhancement of SHG. ${ }^{[142]}$

The effects of strongly bound excitons on the SHG in monolayer TMDs have attracted much attention. ${ }^{[84,136,142]}$ Reference ${ }^{[84]}$ tuned the excitation wavelength from $\sim 680$ to $1080 \mathrm{~nm}$ to study the wavelength dependence of the SHG in monolayer $\mathrm{MoS}_{2}$. It was found that the SHG intensity is significantly enhanced when the SHG wavelength overlaps with the energy of the $\mathrm{C}$ exciton $(\sim 2.8 \mathrm{eV})$, as shown in Figure 3(a). Second-order sheet susceptibility value of $\sim 8 \times 10^{4} \mathrm{pm}^{2} \mathrm{~V}^{-1}$ was reported, when the SHG is in resonance with the $\mathrm{C}$ exciton. ${ }^{[84]}$ For comparison purposes, an effective bulk-like second-order NLO susceptibility is typically obtained by dividing the sheet susceptibility with the thickness of the monolayer (e.g., $0.65 \mathrm{~nm}$ for $\mathrm{MoS}_{2}{ }^{[154]}$ ) yielding an effective bulk-like second-order susceptibility of $\sim 0.1 \mathrm{~nm} \mathrm{~V}^{-1}{ }^{[84]}$ Roughly similar values were also reported for $\mathrm{MoS}_{2}$ in other studies ${ }^{[82,137]}$ as well as for $\mathrm{WS}_{2}{ }^{[87]}$ and $\mathrm{WSe}_{2}{ }^{[91]}$ at the same wavelength range. The tunability of $\chi^{(2)}$ is particularly appealing for many applications. For example, 2DLMs based NLO devices in which the nonlinear conversion processes (e.g., SHG, OPA, and FWM) can be electrically switched on and off could enable new applications in optical signal processing and on-chip ultrafast light generation. As the magnitude of the second-order NLO susceptibility of TMDs is largely related to the exciton resonance condition, the tunability of SHG response in monolayer $\mathrm{WSe}_{2}$ has been demonstrated by using electrostatic doping. ${ }^{[136]}$ In Reference ${ }^{[136]}$, it was shown that the SHG from $\mathrm{WSe}_{2}$ is enhanced by a factor of $\sim 4$ with $160 \mathrm{~V}$ gate voltage change (Figure 3(b)). In addition, reference ${ }^{[142]}$ studied the SHG dispersion in monolayer $\mathrm{MoS}_{2(1-\mathrm{x})} \mathrm{Se}_{2 \mathrm{x}}$ alloys as a function of Se doping (x). By measuring the SHG in a wavelength range between 500 and 900 $\mathrm{nm}$, it was reported that the SHG signal is enhanced at the exciton peak. The results demonstrate the possibility of engineering NLO response of 2DLMs with material composition.

In monolayer $\mathrm{MoS}_{2}$, the two polarization components of SHG depend on the crystal orientation as ${ }^{[82]}: I_{\|}=I_{0} \cos ^{2}(3 \theta)$ and $I_{\perp}=I_{0} \sin ^{2}(3 \theta)$, where $\theta$ is the angle between the mirror plane and the input light polarization, $I_{0}$ is the maximum intensity of the $\mathrm{SHG}^{[82]}$, and $I_{\|}$and $I_{\perp}$ are the SHG intensity components parallel and perpendicular to the input light polarization, respectively. Therefore, SHG can be efficiently utilized to probe the crystallographic orientation of 2DLMs, by measuring the SHG intensity parallel or perpendicular with the excitation polarization direction. ${ }^{[82]}$ 
One of the key challenges in the utilization of NLO effects of 2DLMs is the reduced lightmatter interaction length at the atomic thickness scale, limiting the conversion efficiency. ${ }^{[19,82-}$ ${ }^{84,87,91,137,142]}$ In Reference ${ }^{[83]}$, SHG conversion efficiency in $\mathrm{MoS}_{2}$ (at the $810 \mathrm{~nm}$ excitation) of approximately $10^{-7}$ was measured with the fundamental pump peak intensity of $\sim 10 \mathrm{GW} \mathrm{cm}^{-}$ 2. In Reference ${ }^{[136]}$, conversion efficiency in $\mathrm{WSe}_{2}$ (at the $1470 \mathrm{~nm}$ excitation) of $\sim 4 \times 10^{-10}$ was measured with the fundamental pump peak intensity of $\sim 24 \mathrm{GW} \mathrm{cm}{ }^{-2}$. Note that the conversion efficiency of $\sim 4 \times 10^{-10}$ is large when taking the monolayer thickness into account. For example, it is an order of magnitude larger than what one would achieve if conventional transparent nonlinear crystals are scaled to the same thickness. ${ }^{[2,136,155]}$ For even-order NLO effects (e.g., SHG), another challenge is the fact that they only exist in samples without inversion symmetry (e.g., odd-number of layers of $2 \mathrm{H}-\mathrm{MoS}_{2}{ }^{[95]}$ ). This has been addressed by using static electric fields to break the inversion symmetry in bilayer $\mathrm{MoS}_{2}{ }^{[150]}, \mathrm{WSe}_{2}{ }^{[151]}$ and monolayer graphene ${ }^{[61,65,156]}$ or by utilizing different crystal phases of the same material. ${ }^{[103]}$ In Refs. ${ }^{[103,104]}$, 3R phase of $\mathrm{MoS}_{2}$, which is not centrosymmetric, was shown to produce SHG signal at any layer thickness. By using 3R phase $\mathrm{MoS}_{2}$, the interaction length inside the NLO material can be increased for higher conversion efficiency. Other possible route for increasing the SHG interaction strength is to combine 2DLMs with various optical structures (e.g., waveguides $^{[157]}$, cavities ${ }^{[158,159,160]}$, interference structures ${ }^{[161,162]}$ ). For example, in Reference ${ }^{[157]}$, monolayer $\mathrm{MoSe}_{2}$ was transferred on top of a $220 \mathrm{~nm}$ silicon on insulator slab-waveguide with an increase of around 5 times in SHG conversion efficiency. In Reference ${ }^{[160]}$, continouswave pumped SHG in GaSe was demonstrated with a silicon photonic crystal cavity. More details on how to address the challenges in the utilization of NLO effects of 2DLMs will be discussed in Section 4.10.

\subsubsection{Third harmonic generation}

In contrast to SHG, THG can be possibly generated in materials regardless of whether they are centrosymmetric or not. ${ }^{[1,2]}$ References ${ }^{[45-47]}$ reported on the observation of THG in graphene. For instance, Reference ${ }^{[47]}$ obtained a $\chi^{(3)}$ value of graphene by comparing the THG signal from graphene to that of $\mathrm{SiO}_{2}$ based substrates (e.g., fused silica), reporting that $\chi^{(3)}$ of graphene $\left(\sim 10^{-19} \mathrm{~m}^{2} \mathrm{~V}^{-2}\right)$ is nearly 3 orders of magnitude larger than that of fused silica $\left(\sim 2.5 \times 10^{-22} \mathrm{~m}^{2}\right.$ $\left.\mathrm{V}^{-2}\right) \cdot{ }^{[1,2]}$ Note that the reported $\chi^{(3)}$ values of graphene measured with various excitation wavelengths range from $\sim 10^{-15} \mathrm{~m}^{2} \mathrm{~V}^{-2}{ }^{[45,49]}$ to $\sim 10^{-19} \mathrm{~m}^{2} \mathrm{~V}^{-2} \cdot{ }^{[96,163]}$ Such large deviation between the measured values reflects the challenge in accurate measurement of optical 
nonlinearity of nanomaterials. On the other hand, the large deviation of the measured $\chi^{(3)}$ values in graphene also highlights that the optical nonlinearity in graphene and other 2DLMs can be very sensitive to various parameters (e.g., substrates ${ }^{[71,162,164]}$, defects ${ }^{[165]}$, doping ${ }^{[164]}$, strain ${ }^{[166]}$, chemical treatment ${ }^{[97]}$ ). Despite these deviancies, it is clear that 2DLMs possess large third-order susceptibilities potentially for a wide range of applications. Other 2DLMs (e.g., $\mathrm{MoS}_{2}{ }^{[95-97]}$ in Figure 3(c), $\mathrm{BP}^{[114,115,141]}$ and $\mathrm{GaSe}^{[99]}$ ) have also been shown to possess $\chi^{(3)}$ in the same range as graphene for relatively large THG. Worth noting that Reference ${ }^{[95]}$ found that THG in $\mathrm{MoS}_{2}$ is around 30 times stronger than the SHG (Figure 3(c)). In addition, it has been demonstrated that THG can be used as a rapid and large-area characterization method for determining the crystallographic orientations of exfoliated $\mathrm{BP}{ }^{[114]}$ and the grain boundaries of chemical vapor deposited TMDs. ${ }^{[97]}$

\subsubsection{High harmonic generation}

High harmonic generation can provide an alternative light source in the deep ultraviolet or even the X-ray spectral range. Indeed, the HHG method has been widely used to produce pulses with attosecond $^{[167]}$ (or even zeptosecond ${ }^{[168]}$ ) durations for various fundamental research experiments (e.g., monitoring electronic motion inside materials). Traditionally, HHG is observed from atomic gases ${ }^{[169]}$ and more recently from various bulk crystals (e.g., $\mathrm{ZnO}^{[170]}$, and $\mathrm{GaSe}^{[171]}$ ). However, the underlying mechanism of HHG in bulk solids is still under debate. $^{[101]}$

HHG was experimentally observed in graphene ${ }^{[54]}$ and $\mathrm{MoS}_{2} \cdot{ }^{[101]}$ For example, Reference ${ }^{[54]}$ studied the $5^{\text {th }}, 7^{\text {th }}$ and $9^{\text {th }}$ harmonics in graphene, and observed that HHG signal in graphene is dramatically enhanced under elliptically polarized excitation. It has been found that the $7^{\text {th }}$ and $9^{\text {th }}$ harmonics are significantly enhanced with elliptical polarization compared with the linear polarization excitation result. It was suggested that the HHG mechanism in solids can be characterized by the ratio between the bandgap and Rabi frequency. Due to the zero-bandgap of graphene, the HHG in graphene is in the semimetal regime where the efficiency of the HHG reaches a maximum value at a certain finite pump field ellipticity. In this case, the perpendicular polarization components with respect to the major axis of the pump laser are strongly enhanced. ${ }^{[54]}$

In contrast to graphene, $\mathrm{MoS}_{2}$ has a finite bandgap and thus the mechanism of HHG in $\mathrm{MoS}_{2}$ (and possibly other TMDs) is different from that in zero-gap graphene. ${ }^{[54]}$ Reference ${ }^{[101]}$ studied the HHG in $\mathrm{MoS}_{2}$ with an intense mid-IR excitation $(0.3 \mathrm{eV})$. Figure 3(d) shows the 
HHG spectra generated in $\mathrm{MoS}_{2}{ }^{[101]}$ which reveals that several harmonics up to the $13^{\text {th }}$ order emerge in the visible range. It was found that the odd-order harmonic efficiency per layer exhibits significant enhancement compared to the bulk, which is attributed to the electronic correlation effects. ${ }^{[101]}$ It was proposed that due to reduced dielectric screening in atomically thin semiconductors, the electron-hole interactions would be enhanced. Therefore, the oscillator strength of the strongly bound excitons would increase, enlarging the probability of the electron-hole recollision process. The electron-hole recollision could then be a source of HHG in atomically thin semiconductors, analogous to electron-ion recollision in atomic gases. ${ }^{[101]}$ Reference ${ }^{[172]}$ demonstrated that various underlying wave packet dynamics (e.g., collision, pair annihilation, quantum interference and dephasing) are detected in high-order spectral sidebands in a 60-nm-thick sheet of $\mathrm{WSe}_{2}$ (and also monolayer $\mathrm{WSe}_{2}{ }^{[173]}$ ). HHG with odd orders up to 47 was reported with a THz-driven scheme in the same $\mathrm{WSe}_{2}$ flake. The results indicate that lightwave-driven charge transport can be utilized for attosecond pulse generation with 2DLMs. Nevertheless, the key findings of these proof-of-principle HHG results (including strong fourth harmonic generation ${ }^{[95]}$ ) observed in monolayer ${ }^{[54,101,173,174]}$ and thick-layer ${ }^{[172]}$ 2DLMs suggest that much more research efforts are likely to come in the near future, which will fully discover the possible mechanisms behind the HHG emission in various atomically thin 2DLMs.

\subsection{Four wave mixing}

Four wave mixing is a third-order NLO phenomenon, in which interaction between light beams at two or three different wavelengths occurs to produce one or two new wavelengths. FWM is widely employed for various applications, such as wavelength conversion, optical signal amplification, imaging and many other light manipulation applications. ${ }^{[1,2]}$ Recently, various 2DLMs (e.g., graphene ${ }^{[49,50,175]}, \mathrm{MoS}_{2}{ }^{[93]}, \mathrm{MoSe}_{2}{ }^{[102]}$, and $\mathrm{BP}^{[116,117]}$ ) have been utilized for FWM based applications (e.g., signal processing ${ }^{[50,116,148,176]}$ ).

Graphene based FWM was first reported in the near-infrared region. ${ }^{[49]}$ Figure 4(a) shows the measured NLO response of monolayer graphene as a function of the emission wavelength for several combinations of pump wavelength $\lambda_{1}$ and $\lambda_{2}$. As discussed already above, the values determined for $\chi^{(3)}$ of graphene vary significantly from $\sim 10^{-15}$ to $\sim 10^{-19} \mathrm{~m}^{2} \mathrm{~V}^{-2} \cdot[45,49,96,163]$ Nevertheless, the $\chi^{(3)}$ of graphene is still large, when the atomically thin nature of the material is taken into account. This is usually attributed to the coherence of NLO processes with the strong interband electron transitions. ${ }^{[49]}$ 
The FWM induced NLO effects in TMDs were first investigated from $\mathrm{MoS}_{2}$ thin films and $\mathrm{MoS}_{2}$-graphene heterostructures with NLO microscopy. ${ }^{[93]}$ Figure 4(b) shows the FWM spectrum obtained from a tri-layer $\mathrm{MoS}_{2}$ thin film by introducing pump-probe laser beams at different wavelengths. ${ }^{[93]}$ The FWM imaging indicates that the total FWM intensity is linearly proportional to the number of $\mathrm{MoS}_{2}$ layers ${ }^{[93]}$, possibly attributed to the sum of incoherent contributions from each individual atomic layer (Figure 4(c)). ${ }^{[93]}$ Considering that the SHG intensity from TMDs displays an even-odd oscillation and decays ${ }^{[95]}$ with increasing flake thickness, FWM provides an easy method to measure the film thickness of few-layer $\mathrm{MoS}_{2}$ and other TMD films, similar to other third-order NLO responses (e.g., $\mathrm{THG}^{[95]}$ ).

By integrating 2DLMs with various photonic structures (e.g., photonic crystal cavities ${ }^{[50]}$, microring resonators ${ }^{[177]}$, microfibers ${ }^{[51,178]}$ ), FWM based NLO devices have been realized. For example, high $Q$-factor silicon photonic crystal microcavity has been used to demonstrate cavity-enhanced FWM in graphene, as shown in Figure 4(d). ${ }^{[50]}$ The conversion efficiencies were observed up to $10^{-3}$ at a cavity $Q$-value of 7,500 with low pump power of $600 \mu \mathrm{W} .^{[50]}$ Reference ${ }^{[148]}$ demonstrated a FWM based wavelength conversion by transferring graphene on the end-face of the fiber. A 3-dB tuning range of around $12 \mathrm{~nm}$ was obtained with a peak conversion efficiency of $2 \times 10^{-3}$ for 10 -Gbit s ${ }^{-1}$ non-return-to-zero signal processing. ${ }^{[148]}$ Due to its ultra-high nonlinearity and broad operation bandwidth, graphene-coated microfiber enables multi-order cascaded FWM, potentially applicable for supercontinuum generation and frequency comb applications. ${ }^{[51]}$

More recently, ultrafast optical switching with FWM in BP was investigated (Figure 4 (e)). ${ }^{[116]}$ FWM induced wavelength conversion was enhanced by depositing BP on a D-shaped optical fiber, with an estimated conversion efficiency of $\sim 10^{-7}$. It verified that BP is able to perform ultrafast optical switching at high speed up to $20 \mathrm{GHz}$. Considering the environmental instability of BP, few-layer BP was synthesized ${ }^{[117]}$ with metal-ion-modification against oxidation and degradation ${ }^{[117]}$, providing a new effective option for photonic applications toward high performance and enhanced stability.

\subsection{Saturable absorption}

At present, ultrafast lasers are widely used for numerous applications, ranging from basic research to telecommunication, medicine, and industrial material processing. ${ }^{[5,179]}$ These lasers with output pulse duration in picosecond or femtosecond timescale can be constructed by placing a saturable absorber inside a laser cavity. While the real part of the third-order NLO 
susceptibility (i.e., $\operatorname{Re}\left(\chi^{(3)}\right)$ ) is responsible for typical third-order NLO processes (e.g., FWM and THG), the imaginary part (i.e., $\left.\operatorname{Im}\left(\chi^{(3)}\right)\right)$ dominates the nonlinear absorption such as multiphoton absorption, optical limiting and SA property. ${ }^{[155]}$ Note that higher-order susceptibility (e.g., $\operatorname{Im}\left(\chi^{(5)}\right)$ ) can also contribute to SA. In principle, most light-absorbing materials can be used as saturable absorbers in their resonant absorption wavelength range, which are widely utilized in the passively Q-switched and mode-locked lasers as well as all-optical modulators. ${ }^{[28,30,58]}$ For SA materials, typical macroscopic saturable absorber parameters are the operating wavelength range, the saturable fluence (or intensity), the non-saturable loss, the modulation depth and the recovery time. ${ }^{[28,30]}$

In the past decade, the demonstration of graphene based saturable absorbers has created a new paradigm for building ultrafast pulsed lasers. ${ }^{[23-25,29,30]}$ Figure 5(a) shows SA in graphene at different pump wavelengths, which indicates its broadband operation property. A typical graphene integrated fiber laser is shown in Figure 5(b). More recently, other 2DLMs have also been demonstrated for saturable absorbers (e.g., $\mathrm{MoS}_{2}{ }^{[80,81,180,181]}, \mathrm{WS}_{2}{ }^{[181,182]}, \mathrm{WSe}_{2}{ }^{[183]}$, $\left.\mathrm{MoTe}_{2}{ }^{[181,184]}, \mathrm{WTe}_{2}{ }^{[184]}, \mathrm{ReS}_{2}{ }^{[185]}, \mathrm{BP}^{[111,119,186]}\right)$. Thus far, 2DLMs have been integrated in various lasers (such as solid-state ${ }^{[187]}$, waveguide ${ }^{[188]}$, fiber ${ }^{[23,25,29,30,43,189,190]}$, and semiconductor $^{[191]}$ lasers) to generate ultrashort optical pulses.

Intensive research efforts on 2DLMs based SAs have demonstrated that 2DLMs can provide cost-effective saturable absorbers with superior performance for compact ultrafast lasers. For example, with the help of the broadband absorption of graphene, a single saturable absorber can realize mode-locking operation with an operation bandwidth of up to $>1000 \mathrm{~nm} .{ }^{[44]}$ Broad operation bandwidth of graphene was also demonstrated by the wide wavelength-tunable output (Figure 5(c)). ${ }^{[43,189]}$ Thus far, 2DLMs based ultrafast lasers have been reported to produce mode-locked pulses with the output wavelength covering from $635 \mathrm{~nm}^{[181]}$ to $2.8 \mu \mathrm{m}^{[30]}$, pulse duration of sub 20-fs ${ }^{[192]}$ (Figure 5(d)), the repetition rate of $>10 \mathrm{GHz}^{[193]}$, and the average power of $>10 \mathrm{~W} .{ }^{[194]}$ Overall, the advantages of 2DLMs based saturable absorbers include broad operation bandwidth ${ }^{[44]}$, fast recovery time ${ }^{[60,80]}$, electrical tunability ${ }^{[12,74]}$, costeffective, easy fabrication and integration ${ }^{[30]}$, and multi-functionality (e.g., integrated photodetector and modulator $\left.{ }^{[12]}\right)$. For example, a recent example of adding additional functionalities to the lasers was demonstrated in Reference ${ }^{[111]}$, which utilized the anisotropic optical properties of BP for linearly polarized ultrafast pulse generation with a degree of polarization of $\sim 100 \%{ }^{[111]}$ (Figure 5 (e)). The SA property can also be used for all-optical 
modulation. ${ }^{[31]}$ Due to their ultrafast recovery time, 2DLMs based all-optical modulators may operate significantly faster than their electrical counterpart. ${ }^{[28,195]}$ Reference ${ }^{[28]}$ demonstrated a graphene integrated all-optical fiber modulator with $\sim 200 \mathrm{GHz}$ bandwidth (Figure 5 (f)), which clearly shows high potential of 2DLMs for ultrafast signal processing. In addition, higher modulation depth can be potentially achieved with TMDs than graphene in the visible spectrum range due to their strong absorption in the visible spectrum range. ${ }^{[81]}$

\section{Perspectives of 2DLMs based nonlinear optics}

\subsection{Fundamental limits of nonlinear optics}

The fundamental physical limits of nonlinear optics have long been theoretically discussed not only to determinate the technological potential of nonlinear optics, but also to enable a wide perspective on the current NLO devices for performance improvement. ${ }^{[196]}$ 2DLMs are the ideal material family to address the limits due to their atomically-thin nature. For example, it will be very beneficial to fully understand how the material properties of 2DLMs affect their NLO responses. Currently, it is still hard to precisely calculate the properties of new nanomaterials. Additionally, it can be very difficult to accurately measure the properties of new nanomaterials that can be highly susceptible to environmental disturbances (e.g., substrates $[71,122,164]$, defects ${ }^{[165]}$, doping ${ }^{[164]}$, strain ${ }^{[145,166]}$, chemical and laser treatments ${ }^{[97]}$ ). For graphene $^{[197]}$, even now in 2017, after various NLO properties (e.g., SHG in bilayer and multilayer graphene ${ }^{[61,65,66,198]}, \mathrm{THG}^{[47]}, \mathrm{FWM}^{[49]}, \mathrm{SA}^{[23-25]}$, and optical limiting ${ }^{[56]}$ ) have been extensively investigated, a long-running discrepancy still exists between some of the experimental observations (e.g., graphene nonlinear susceptibility ${ }^{[163,199]}$ ). For example, there is a huge variation in measured parameters of graphene $\mathrm{SA}^{[25,29-31]}$ and $\mathrm{THG}^{[47,49,95]}$ (Listed in Table 1), even though they are the most comprehensively studied NLO properties of graphene. Further, the measured NLO coefficients of other 2DLMs also differ a lot from each other (e.g., $\sim 3$-order of magnitude difference ${ }^{[82-84,200-202]}$ in $\chi^{(2)}$ of $\mathrm{MoS}_{2}$, ranging from 0.1 to $100 \mathrm{~nm} \mathrm{~V}^{-1}$ [82-84,200,201], Table 1). In this regard, more reliable and accurate characterization and calculation methods are needed. Note that the methods and the figures of merit that are used for characterization of traditional bulk NLO crystals are not always directly applicable to 2DLMs. For example, the traditional definition of nonlinear susceptibility involves polarization per unit volume $^{[1,2]}$, which is an ill-defined figure-of-merit for 2DLMs since these materials contain only surface. ${ }^{\left[{ }^{95]}\right.}$ Therefore, in certain cases, it might be more appropriate to compare the NLO 
processes in 2DLMs with the ratio between the harmonic signal power and the incident pump power (i.e., harmonic conversion efficiency). ${ }^{[95]}$

Nevertheless, it is of great significance to carefully measure the NLO responses of various 2DLMs to fully understand the fundamental physics of their NLO properties. This can be associated with advance theoretical techniques to quantitatively understand various NLO

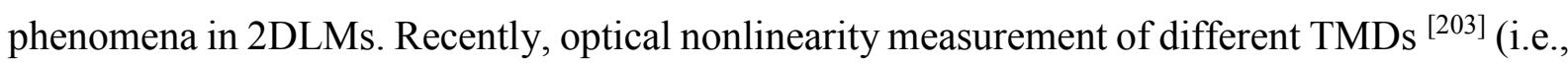
monolayer and few-layer $\mathrm{MoS}_{2}, \mathrm{MoSe}_{2}, \mathrm{WS}_{2}, \mathrm{WSe}_{2}$, graphene, BP, Figure 6) has been simultaneously carried out on a substrate. The typical $\chi^{(2)}$ and $\chi^{(3)}$ of different monolayers are listed in Figure 6. This constitutes the first opportunity for an accurate and detailed comparison of NLO responses of 2DLMs in a controlled experimental environment. ${ }^{[203]}$ The results reveal feasibility of linking the linear and NLO responses of different 2DLMs ${ }^{[203,204]}$ to predict responses of unknown 2DLMs. In the future, it is anticipated that a large range of 2DLMs will be further inspected on their physical properties (including NLO properties), which will then be combined with a theoretical framework to substantially advance our understanding of 2DLMs. The short-term goal is to identify unknown trends to predict, understand and differentiate physical properties (including NLO properties) of 2DLMs. This will lead to efficiently discover 2DLMs with high performance (including high optical nonlinearity) from the family of hundreds of new different 2DLMs that have not been touched in the lab so far. It is worth noting that this can significantly benefit the whole nanomaterial research community in its effort to understand the extraordinary structures and functions (e.g., NLO properties) of various nanomaterials (including quantum dots, nanotubes, nanowires) beyond 2DLMs. The ultimate goal is to artificially create new nanomaterials for devices with superior performance (e.g., ultra-high NLO response).

\subsection{D material characterization}

Material characterization is a scientifically and technologically important task to provide relevant information about the properties of the materials that enable the state-of-the-art devices to function. However, currently, it is challenging to accurately measure various physical properties of nanomaterials (including atomically-thin 2DLMs). As explained in Section 3, the intensities of SHG and THG signals are much higher in 2DLMs than that generated in the substrate, producing a high contrast between the substrate and the materials. It demonstrates that various NLO processes allow the measurement of various properties of 2DLMs. ${ }^{[45,97,114]}$ This is well aligned with the recent efforts of using various NLO processes 
(e.g., multiphoton processes ${ }^{[11]}$, Raman, FWM) for characterization of biological samples. Reference ${ }^{[97]}$ observed 4 different grain boundaries in CVD grown $\mathrm{MoS}_{2}$ monolayers by mapping the SHG and THG signal intensities (Figures 7(a) and 7(b)). The angle between crystal axes on neighbouring grains (i.e., $\mathrm{A}_{1}$ and $\mathrm{A}_{2}$, and $\mathrm{B}_{1}$ and $\mathrm{B}_{2}$ ) is extremely small, $\sim 1^{\circ}$, making these grain boundaries irresolvable with Raman measurements. SHG measurements, which are known for their sensitivity to deduce the crystal orientations and grain boundaries [82-84,139,200-202,205], also fail to resolve these grain boundaries due to the small difference between the crystal axes. In this case, Reference ${ }^{[97]}$ showed that THG can be more effective to detect these grain boundaries. Further, the crystallographic directions of exfoliated 2D material flakes (e.g., BP) have been recently characterized by using multiphoton microscopy, see Figures 7(c) and 7(d). ${ }^{[114]}$ As observed, the crystallographic directions of the BP thin films are clearly measured by observing the THG signal intensity as a function of the excitation laser polarization direction. ${ }^{[14]}$ The layer thickness of the 2D material flakes can also be evaluated from the intensity of the multiphoton signal. ${ }^{[45,114]}$ The advantage of nonlinear multiphoton microscopy is that it is extremely straightforward and very fast. It was noted that an area of 450 $\times 450 \mu \mathrm{m}^{2}$ can be imaged with a multiphoton microscope in about $5 \mathrm{~s}$ or less, highlighting its speed advantage compared to other commonly used characterization techniques (e.g., Raman, PL). The accuracy and the speed of the multiphoton microscopy make it a very useful characterization tool for characterization of 2DLMs (especially CVD grown large-scale $2 \mathrm{DLMs}^{[97]}$ ) in the near future.

Further, it is well-known that various structural defects (e.g., vacancies, adatoms, grain boundaries, edges) in the materials and external environments (e.g., substrate, strain) around the materials can significantly affect the NLO responses of the materials. ${ }^{[1,2]}$ This also has been experimentally observed in 2DLMs (e.g., effects of substrates ${ }^{[162]}$, defects ${ }^{[165]}$, doping ${ }^{[71,164]}$, strain ${ }^{[145,166]}$, and chemical and laser treatments $\left.{ }^{[97]}\right)$. These results have shown that nonlinear optics can be potentially used to characterize the properties of 2DLMs. On the other hand, these effects can be utilized to engineer the NLO responses in 2DLMs. For example, the structure defects can be beneficial by trapping carriers and excitons, enabling unprecedented functionalities (e.g. defect induced single photon generation ${ }^{[123,206]}$ ). This can possibly introduce new NLO phenomena (e.g., multi-photon excited single photon emission, all-optical nonlinear switching with only one or a few photons). 


\subsection{Bio-imaging and sensing applications}

NLO imaging (e.g., SHG and two-photon luminescence imaging methods) has attracted wide attention in both fundamental research and biomedical applications, due to its good biocompatibility, large imaging depth, superior spatial resolution, minimized tissue fluorescence background, and the reduction of photo-damage in biological tissues. ${ }^{[1]}$ For example, in contrast to the one-photon (i.e., linear optical) processes based luminescence imaging methods, near-infrared excitation (700-1350 nm) based two-photon luminescence imaging approach can enable larger penetration depth (even up to $1800 \mu \mathrm{m}$ ) into tissues, significantly pushing the fundamental imaging penetration depth limit. ${ }^{[207]}$ Reference ${ }^{[208]}$ first used graphene oxide as a non-bleaching optical probe in biomedical diagnostics. The result shows that functionalized graphene oxide can be employed for three-dimensional two-photon luminescence imaging in laser-based cancer micro-surgery. ${ }^{[208]}$ Two-photon luminescence of nitrogen-graphene quantum dots was also investigated with femtosecond laser excitation and applied for two-photon cellular imaging. ${ }^{[207,209]}$ Similarly, it was demonstrated that S6 ribonucleic acid aptamers conjugated graphene oxide can be used for selective two-photon imaging of SK-BR-3 breast tumour cell (see Figure 8(a)). ${ }^{[210]}$ Besides graphene, $\mathrm{MoS}_{2}$ quantum dots have been reported for bio-imaging by up- and down-conversion $\mathrm{PL}^{[211]}$ and multiphoton emission. ${ }^{[212]}$ The method combining good biocompatibility and physiological stability of $\mathrm{MoS}_{2}$ quantum dots enables high-performance bio-imaging of HeLa cells, as shown in Figure 8(b). ${ }^{[211]}$ As demonstrated by these pioneering studies on bio-imaging with graphene and $\mathrm{MoS}_{2}, 2 \mathrm{DLMs}$ are very promising candidates for non-invasive bio-imaging applications. ${ }^{[213]}$ Note that other NLO effects in 2DLMs (e.g., FWM, OPG) also can be used for bio-imaging applications.

Nowadays, sensors are used almost everywhere and play an important role in our lives. Owing to the atomically thick interaction length, the NLO interaction between light and 2DLMs needs to be further enhanced for practical sensing applications. By utilizing polariton in 2DLMs (e.g.,

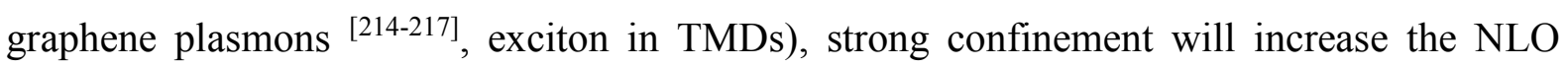
response by orders of magnitudes, potentially enabling practical sensing applications. ${ }^{[218]}$ In Reference ${ }^{[218]}$, nano-graphene can serve as optical sensors of charge- and dipole-carrying analytes with single-molecule sensitivity. An elementary charge or a weak permanent dipole carried by the molecule was shown to be sufficient to trigger observable modifications in the nonlinear response of the graphene nano-islands through atomistic quantum-mechanical 
simulations. In particular, a strong SHG signal, forbidden by symmetry in the unexposed graphene nanostructure, emerges due to a redistribution of carriers produced by interaction with the molecule, as shown in Figure 8(c). ${ }^{[218]}$ These results pave the way toward ultrasensitive NLO detection of dipolar molecules that can be achieved by utilizing the extraordinary NLO properties of graphene and other 2DLMs. In principle, other approaches to

enhance NLO response in 2DLMs, such as optical cavities ${ }^{[50,158]}$, waveguides ${ }^{[157]}$ (e.g., slot waveguides $^{[219]}$, photonic crystal fibers ${ }^{[12]}$ ) (discussed in Section 4.10) can also be utilized for bio-imaging, sensing and other enabling applications, deserving further investigation in the near future.

\subsection{THz wave technology}

Terahertz radiation (from $\sim 0.1$ to $10 \mathrm{THz}$ ) has the ability to pass through a wide range of substances, thus possessing numerous potential applications in biomedical imaging, security, remote sensing and spectroscopy. ${ }^{[220]}$ However, present THz technologies seriously suffer from the lack of compact and efficient room temperature $\mathrm{THz}$ sources and detectors. This greatly limits the proliferation of various practical applications. ${ }^{[220]}$

Research on THz technologies with 2DLMs has attracted huge interest. In contrast to numerous fascinating theoretical concepts proposed on $\mathrm{THz}$ generation in graphene ${ }^{[221]}$, currently, main experimental demonstrations still concentrate on bulk materials (e.g., thick-layer graphite ${ }^{[222]}$, topological insulators ${ }^{[223]}$, thick-layer $\mathrm{MoS}_{2}{ }^{[224]}$ and $\mathrm{WS}_{2}{ }^{[225]}$ ). There are only few experimental demonstrations with monolayer and few-layer graphene for ultrafast, broadband, and cost-effective $\mathrm{THz}$ light emission..$^{[55,226,227,228]}$ For example, experiments on graphene excited by femtosecond optical pulses at oblique incidence (Figure 9(a)) have shown second order nonlinear photon-drag-currents associated with $\mathrm{THz}$ generation at room temperature. ${ }^{[226,228]}$ The photon drag effect can induce carriers driven by in-plane surface field into transient current (Figure 9(b)). It generates THz waves ranging from 0.1 to $4 \mathrm{THz}$ with an optical-to-THz conversion efficiency ${ }^{[226]}$ of $\sim 1.5 \times 10^{-11}$, indicating a second-order nonlinear process (Figures 9(c) and (d)). The photon drag induced $\mathrm{THz}$ generation can be enhanced by surface plasmon excitation ${ }^{[229]}$ and modulated by applying gating ${ }^{[230]}$, showing the potential of 2DLMs based efficient THz sources.

Different from transient photocurrent induced $\mathrm{THz}$ generation in graphene, the underlying mechanism of $\mathrm{THz}$ generation in $\mathrm{MoS}_{2}$ can be surface optical rectification under reflection configuration. ${ }^{[224]}$ Few-layer $\mathrm{MoS}_{2}$ can generate $\mathrm{THz}$ radiation efficiently ranging from 0.1-3.5 
$\mathrm{THz}$ under linearly polarized femtosecond laser excitation $(800 \mathrm{~nm}$ central wavelength, $35 \mathrm{fs}$ pulse duration). $\mathrm{THz}$ wave amplitude generated in $\mathrm{MoS}_{2}$ is quadratic with the pump electric field, also indicating the generation origin from a second-order nonlinear effect (i.e., optical rectification). The investigations on layered $\mathrm{MoS}_{2}$ can not only deepen the understanding of NLO rectification process but also potentially provide a sensitive and noninvasive method to characterize the surface and interface properties of $\mathrm{MoS}_{2}$ by $\mathrm{THz}$ surface emission spectroscopy.

Currently, the THz light intensity generated in graphene and other 2DLMs (e.g., $\mathrm{MoS}_{2}$ ) is still not strong enough for practicable $\mathrm{THz}$ sources mainly due to their atomically thin interaction length. Full potential of 2DLMs for $\mathrm{THz}$ generation still deserves further exploration by designing novel approaches (for example, stacking multiple monolayers, heterostructures, evanescent mode integration) or coupling with graphene plasmons ${ }^{[231]}$, optical cavities, waveguides or antennas $^{[232]}$ (as discussed in Section 4.10). Further, NLO processes are one of the most dominant methods to detect THz light. ${ }^{[220]}$ In this regard, 2DLMs with their relatively large optical nonlinearity can be potentially used for integrated $\mathrm{THz}$ light detections.

\subsection{Quantum photonics}

Quantum photonics is the research field that utilizes semi-classical or quantum physics to understand light-matter interactions. ${ }^{[233]}$ Potential applications of quantum photonics include large-scale secure data transfer systems, quantum computers and efficient search engines for databases. ${ }^{[233]}$ 2DLMs based nonlinear optics may find new applications in quantum photonics. Thus far, the field of 2DLMs based quantum photonics has mainly been focusing on the linearly operated single photon sources (SPSs) which emit only one photon per excitation process. Current state-of-the-art SPSs use nonlinear crystals ${ }^{[234]}$, atom traps ${ }^{[235]}$, color centers of diamond $^{[236]}$, and semiconductor quantum dots. ${ }^{[237]}$ Recently, 2DLMs based SPSs have been demonstrated using two different concepts: emission via localized defects (e.g., in h-BN crystals ${ }^{[123,124,206]}$ ) and via localized strain fields (e.g., in TMDs ${ }^{[123,206]}$ ). In general, the emission mechanism can be explained by linear optics. However, from the nonlinear quantum photonics perspective, entangled photon pairs (EPPs) have been of particular interest in the field of quantum photonics. In fact, EPP is one of the most important building blocks of quantum technologies (e.g., the fabrication of Einstein-Podolsky-Rosen type photon pairs). During the last few decades, efficient means have been searched to create EPPs by utilizing spontaneous down-conversion process ${ }^{[238]}$ and Kerr nonlinearity induced spontaneous FWM 
${ }^{[239]}$ in NLO materials. The strength of these NLO effects depends on the second and third order NLO susceptibilities, respectively. As a result, a larger optical nonlinearity can enable a more effective way for realization of the EPPs. However, down-scaling of the currently widely used bulk crystals into PICs would be important for future quantum technologies but has so far been proven challenging due to difficulties in integrating the traditional NLO bulk materials in PICs for on-chip applications. Therefore, 2DLMs (e.g., graphene, BP, GaSe, $\mathrm{MoS}_{2}$, etc), that can easily be integrated in PICs may find applications in the field of quantum photonics (e.g., spontaneous down-conversion and FWM based quantum light sources, manipulation and storage of quantum states of light in integrated circuits). As an example of this perspective, we note the recent theoretical predictions which postulate that graphene plasmonic nanostructures can combine the strong confinement of optical fields and the large intrinsic optical nonlinearity in graphene for EPPs ${ }^{[63]}$ and NLO interactions at the few photon level. ${ }^{[240]}$

\subsection{Attoscience and strong field physics}

Attoscience generally refers to the utilization of attosecond $\left(\sim 10^{-18} \mathrm{~s}\right)$ pulses of particles (e.g., electrons or photons) in the investigation and manipulation of the ultrafast dynamics in materials. ${ }^{[241]}$ For this purpose, pulsed lasers with the duration in the attosecond scale (or even in the zeptosecond scale) are required. Thus far, attosecond pulses have been mainly created via HHG processes. ${ }^{[170,242]}$ In fact, if the HHG process involves only virtual levels, the NLO response is in theory immediate, and therefore could occur at a time scale of sub-femtosecond (as demonstrated in bulk crystals ${ }^{[170]}$, and argon and krypton gas jets ${ }^{[242]}$ ). Recently, HHG signal has been observed in 2DLMs ${ }^{[54,101,172,173]}$ (discussed in Section 3.1.3). Although it should be noted that the duration of the generated pulses has not thus far been measured, the observation of $\mathrm{HHG}^{[101]}$ in atomically thin 2DLMs demonstrates the ability to study strongfield and attosecond phenomena in materials of reduced dimensionality. ${ }^{[101,216]}$

Furthermore, recent demonstrations have shown that strong-field driven phenomena with nanoscale structures typically occur in sub-cycle duration, which has been widely explored to understand the collective electron dynamics and generate optical-field-driven currents on attosecond timescale. ${ }^{[243]}$ Reference ${ }^{[244]}$ shows that current induced in graphene by few-cycle optical pulses can be controlled with a precision on the attosecond time scale. This indicates that field-driven processes, such as HHG in graphene and other 2DLMs, can be very interesting for various new attoscience applications (e.g., sub-cycle photoemission, attosecond metrology, attosecond control of electronic processes). 


\section{WILEY-VCH}

\subsection{Broadband light sources}

A possible field where 2DLMs may also find new applications is broadband light generation (e.g., ultraviolet (UV) and infrared light generation). With suitable 2DLMs, realization of compact photonic circuits with on-chip UV light sources would find various applications, e.g., in sensing, light therapy ${ }^{[245]}$ and photolithography. ${ }^{[246]}$ However, transparent materials with large NLO susceptibility in the UV region are hard to find, the UV cut-off edge of most materials lies well above $200 \mathrm{~nm}$. Based on the transparency advantage, $\mathrm{h}-\mathrm{BN}^{[247]}$ and other large-bandgap 2D material insulators could be suitable candidates for UV generation around $200 \mathrm{~nm}$ via various NLO processes (e.g., harmonic generation, SFG ${ }^{[93,248]}$, Raman and $\mathrm{FWM}^{[12]}$ ). To our best knowledge no such demonstrations yet exist and thus the utilization of 2DLMs for UV light generation still remains elusive. In addition, BP and other narrow bandgap 2DLMs can be potentially used for infrared light generation (e.g., via harmonic generation and differential DFG). It is worth noting that white light emission in graphene has been demonstrated with continuous wave ${ }^{[249]}$ or ultrafast pulsed ${ }^{[250]}$ light pumping. Photon emission generates typically throughout the visible spectral range with energies both larger and lower than the exciting one, in contrast with conventional PL processes. ${ }^{[26]}$ Such NLO processes (e.g., avalanche multiphoton ionization ${ }^{[249]}$, inter-valence charge transfer ${ }^{[249]}$ ) can also be utilized for construction of new types of broadband light sources (e.g., white, UV or infrared light generation).

\subsection{Optical parametric generation}

Optical parametric generation is a NLO process that commonly uses $\chi^{(2)}$ of NLO crystals to amplify an optical signal (i.e., $\mathrm{OPA}^{[251]}$ ) or directly generate coherent light output (i.e., $\mathrm{OPO}^{[252]}$ ). The challenge of using 2DLMs for OPAs and OPOs is to achieve strong light-matter interaction for efficient light amplification. This is very hard to be directly obtained with monolayer 2DLMs. Reference ${ }^{[253]}$ theoretically reports that the phase-matching-free OPOs are possible with micro-resonators adopting TMDs as gain materials. Note that the second-order NLO effects are only present in TMDs with odd-number of layers, thus TMDs with evennumber of layers cannot be used. Another possible solution is to use 3R phase TMDs, which are non-centrosymmetric with arbitrary layer number. ${ }^{[103,104]}$ Further, using heterostructure lattices also can coherently increase the NLO response.

Another possible route for achieving OPAs or OPOs with 2DLMs could be to utilize FWM based parametric gain. FWM is a third-order NLO process and thus present in any number of 
layers. In Reference ${ }^{[254]}$, FWM from silicon nitride waveguide was used to create an OPO operating at $1550 \mathrm{~nm}$. Similar approach in which 2DLMs are combined with waveguides ${ }^{[157]}$ or cavities could be a potential solution for the construction of an OPO or OPA (discussed in Section 4.10), offering a pathway for the fabrication of CMOS compatible light amplifiers and sources at the telecommunication wavelengths. If successful, 2DLMs based OPOs can offer novel light sources having various distinctive properties (such as wide operation bandwidth, low-threshold, high-gain and dual-wavelength output), different from the previously reported 2DLMs based lasers. ${ }^{[255]}$ Such new parametric devices enabled by 2DLMs will be more compact, stable and cheap than the existing counterparts, suitable for various emerging applications. For example, gate-tunable NLO responses in 2DLMs (e.g., graphene and TMDs) can fully enable electronically tunable integrated light sources (e.g., frequency combs) for metrology, sensing, and on-chip telecommunication.

\subsection{Nonlinear optics with polaritons}

Dipole-type polaritonic excitations (e.g., plasmon-polaritons in graphene ${ }^{[256]}$, phononpolaritons in $\mathrm{h}-\mathrm{BN}^{[33,257]}$, and excitons in TMDs ${ }^{[258]}$ ) play a key role in understanding lightmatter interactions in various 2DLMs and their heterostructures. ${ }^{[33,259]}$ Thus far, the majority of NLO experiments demonstrated with 2DLMs were investigated with pump wavelengths offresonant from these polaritons. In principle, extremely strong NLO responses can be anticipated using a pump with the wavelength resonant to the polaritons. For example, it has been shown that SHG can be strongly enhanced if the excitation photons are resonant with the exciton in 2DLMs. ${ }^{[86]}$ In principle, tightly-confined and low-loss polaritons, such as plasmonpolaritons in graphene and phonon-polaritons in $\mathrm{h}-\mathrm{BN}$, are possible to be utilized to significantly improve the NLO interaction. As theoretically demonstrated, graphene plasmonic resonance can be utilized to enhance the NLO responses (e.g., FWM and HHG) in various graphene nanostructures, see Figure 10(a). ${ }^{[214-216]}$ For a graphene nano-island with a side length of $8.4 \mathrm{~nm}$ and doped with charge $Q=3 \mathrm{e}$, illuminated by two collinear light pulses of central energies $\hbar \omega_{1}=0.41 \mathrm{eV}$ and $\hbar \omega_{2}=0.66 \mathrm{eV}$, extremely high wave-mixing efficiency was theoretically calculated when one or more of the input or output frequencies coincide with the plasmon resonance of graphene nano-islands. ${ }^{[214]}$ By tuning the charge density in graphene nanostructures (e.g., via gating), enhanced nonlinear responses can be realized over a wide spectral range in the visible to near-infrared. ${ }^{[73]}$ Indeed, the enhanced nonlinear mixing of nearinfrared and mid-infrared light was experimentally detected in arrays of graphene 
nanoribbons. ${ }^{[260]}$ Note that intrinsic NLO response can enable all-optical plasmon coupling in graphene with visible light pulses. ${ }^{[231]}$ Huge enhancement of NLO response created by the plasmonic resonance in 2DLMs (e.g., plasmonic excitation of graphene for $\mathrm{THz}$ and midinfrared light generation, modulation and manipulation) can support the exceptional potential of various NLO applications, including novel light sources (e.g., spasers), nonlinear light modulators, light multiplexers, metasurfaces, and sensing devices.

Further, the group velocity of polaritons in 2DLMs and their heterostructures can be exceptionally slow ${ }^{[33,261]}$ with ultra-long propagation lengths and lifetimes. Such unique slow light effects will open up new opportunities for enhancing the NLO interaction between light and matter for various applications (e.g., harmonic generation, pulse compression and soliton generation). ${ }^{[262]}$ For instance, dispersion engineering in 2DLMs with various structures (e.g., nanoribbons, nanodots) can facilitate the generation of soliton pulses at the deepsubwavelength scale, thanks to the combination of graphene's intrinsic self-focusing nonlinearity and graphene plasmonic confinement effects. ${ }^{[263]}$ Therefore, 2DLMs and their heterostructures potentially can introduce new horizons for generation, propagation and manipulation of soliton pulses over short length scales, at low powers and with ultrafast pulses for all-optical signal processing applications.

In monolayer TMDs, the lack of inversion symmetry along with strong spin-orbit coupling leads to the spin-valley coupling. ${ }^{[264,265]}$ One of the intriguing consequences induced by spinvalley coupling is the valley polarization observed in monolayer (also in biased or folded bilayer) TMDs ${ }^{[105,139,266]}$, where the electron-hole pairs in distinct valleys can be selectively excited by circularly polarized light. More recently, the valley selection rule has been demonstrated in various multiphoton processes (e.g., SHG and two-photon luminescence in TMD monolayers $\left(\mathrm{WS}_{2}, \mathrm{WSe}_{2}\right){ }^{[136,267]}$, which is opposite to the one-photon linear optical process. ${ }^{[105,139,266]}$ For excitation with left circular (right circular) photons at the fundamental frequency $\omega$, the SHG photons at frequency $2 \omega$ are right-circularly (left-circularly) polarized with almost $100 \%$ helicity ${ }^{[265]}$ at $+\mathbf{K}(-\mathbf{K})$ points in the Brillouin zone, as shown in Figure 10(b). ${ }^{[136]}$ This might be interesting to combine with various vector beam excitations. ${ }^{[268]}$ Moreover, SHG can be enhanced when the excitation photons are resonant with the A exciton $^{[86]}$, which is also electronically tunable. ${ }^{[136]}$ Therefore, it is expected that the exciton related NLO responses and their valley-dependent properties in 2DLMs and their heterostructures will ignite a new research area worthy of deep exploration. For example, 
strongly coupled cavity exciton-polaritons have been experimentally demonstrated through interaction between bound electron-hole pairs in 2DLMs and the cavity optical field for NLO applications. ${ }^{[269]}$ Topological polaritons and exciton-mediated superconductivities in 2DLMs have also been theoretically predicted. ${ }^{[270]}$ The collective coherence of the quasiparticle nature of these exciton-polaritons in 2DLMs and their heterostructures can provide a new NLO material platform for various intriguing nonlinear quantum phenomena ${ }^{[270]}$ (e.g., crossover between Bose-Einstein and Bardeen-Cooper-Schrieffer condensed states ${ }^{[271]}$ ).

\subsection{Optical nonlinearity enhancement in 2DLMs}

As we mentioned previously, the NLO light-matter interaction builds up coherently with increasing thickness in a conventional NLO crystal (with the efficiency of the interaction typically dictated by the phase-matching conditions ${ }^{[1,2]}$ ). Therefore, even with perfect phasematching, the efficiency of the frequency conversion depends on the effective path length inside the nonlinear material. Since 2DLMs contain only one (or few) atomic layers, the interaction length is limited to (sub-) nanometer scale. Hence, the atomically thin nature of the 2DLMs poses strong limitations on total conversion efficiency for NLO applications. More research efforts are likely being directed in the near future towards the development of optical configurations which enhance NLO interaction. Indeed, several schemes have recently been proposed to improve the light-matter interaction, including phase-matched approaches (e.g., atomically phase-matched approaches ${ }^{[103]}$ or with patterning layered materials ${ }^{[272]}$ ), waveguide integration ${ }^{[111,116]}$ plasmonic enhancement ${ }^{[130,214,216,260]}$ (also mentioned in Sections 4.3 \& 4.9) and optical resonators. ${ }^{[158,160]}$ In particular, by utilizing various photonic resonators (e.g., photonic crystal cavity, Figure 11(a); microsphere cavity, Figure 11(b); microdisk resonators, Figure 11(c), hybrid nanoantennas), it is anticipated that 2DLMs based integrated photonic structures are promising for future NLO applications. In addition, metallic plasmonic polaritons $^{[130,273]}$ (Figure 11(d)) and photonic metamaterials ${ }^{[274]}$, with benefits of easy fabrication, integration and local field enhancement, would be suitable to enhance the optical nonlinearity of 2DLMs. Additionally, electrically tuneable functionality in 2DLMs (e.g., graphene and TMDs) provides a powerful method to tune the generation of NLO signal for practical applications, which is challenging with traditional NLO materials. For example, electrically controlled optical frequency comb generation or OPOs could be achieved by integrating micro-resonators with 2DLMs (Figure 11(e)). This potentially possesses a large range of applications in metrology, sensing and high-capacity telecommunication. ${ }^{[275]}$ 


\section{WILEY-VCH}

\section{Conclusions and outlook}

The major advances of nonlinear optics have enabled the realization of myriad technologies that are playing a crucial role in our modern society. Today, the field of 2DLMs based nonlinear optics is still in its infancy, even though it has gained an increasing amount of attention because it may allow the realization of a vast number of technologically relevant applications that have not been achieved before. For instance, the present $\mathrm{THz}$ technologies suffer from the lack of compact room temperature $\mathrm{THz}$ sources, preventing their usage in biomedical imaging, security, remote sensing and spectroscopy. Recent demonstrations show that ultrafast, broadband, and low-cost THz light emission in 2DLMs is possible by utilizing the NLO effects. Further, 2DLMs and carbon nanotubes ${ }^{[276]}$ based saturable absorbers are one of the best-known examples in the field of nanomaterials based nonlinear optics, which have relatively high levels of readiness for commercialization. All-optical signal processing is another topic in which 2DLMs based nonlinear optics could find practical applications.

Thanks to the recent progresses made in the fabrication processes of 2DLMs, various 2DLMs could be embedded in PICs (e.g., silicon waveguides and other integrated optical circuits) relatively easily and cost-effectively. In addition, 2DLMs can offer a large range of enabling functions $^{[19]}$ (e.g., electronics, energy storage and conversion, sensing, photonic and optoelectronic functions) due to their diverse properties. In this regard, multi-functional optical devices $^{[31]}$ (e.g., multi-functional modulator and photodetector ${ }^{[277]}$, multi-functional modulator and plasmonic waveguide ${ }^{[278]}$ ) have been demonstrated with 2DLMs. This will not only provide a significant advantage for electronics and photonics in terms of possible integration with an "all-in-one" solution, but also offer new devices with superior performance (e.g., simultaneous modulation and detection ${ }^{[277]}$, electronically tunable NLO devices). In principle, similar strategy can be utilized to simultaneously operate 2DLMs based NLO devices for demonstration of devices with other multi-functionalities. Further, as noted above, 2DLMs based nonlinear optics could find applications in attoscience, quantum photonics, UV generation and spin/valleytronics. On top of this, the recent progresses in 2DLMs based bioimaging have also attracted huge attention due to possible performance improvements. In fact, 2DLMs have already been shown to be very promising candidates for non-invasive probes in the near future bio-imaging applications. Benefited from all the listed advantages of 2DLMs, we believe that 2DLMs based nonlinear optics will pave the way for a wide variety of novel applications in various cross-disciplinary fields. Hence, if the production of environmentally 
stable, large-scale and high-quality $2 \mathrm{DLMs}$ is successful ${ }^{[68,119,279]}$, the future of this relatively new field is likely to be bright with the next few years revealing not only new research demonstrations but also the technological applications.

\section{Acknowledgements}

We acknowledge funding from the Academy of Finland (No. 276376, 284548, 295777, 304666, 314810), TEKES (OPEC), the European Union's Seventh Framework Program (No. 631610), Aalto Centre of Quantum Engineering, China Scholarship Council, and the provision of technical facilities of the Micronova, Nanofabrication Centre of Aalto University. Z.S. greatly acknowledges postdocs and students working on the related topics in Aalto since 2013, such as Dr. Antti Säynätjoki, Dr. Lasse Karvonen, Dr. Bo Fu and Dr. Diao Li. Z.S. also benefited greatly from many international collaborators, specifically the groups of Profs. Nasser Peyghambarian, Khanh Kieu, and Robert A. Norwood in University of Arizona; Profs. Andrea C. Ferrari and Tawfique Hasan from Cambridge University; Prof. Roy Taylor and Dr. Edmund Kelleher from Imperial College London; Profs. Mark C. Hersam and Kim Fook Lee from Northwestern University; Prof. Xianhui Chen from University of Science and Technology of China; Prof. Goki Eda and Dr. Shisheng Li from National University of Singapore; Dr. Marco Polini from Istituto Italiano di Tecnologia, Italy; Prof. Javier García de Abajo from the Institute of Photonic Sciences, Spain; Prof. Kaihui Liu from Peking University; Profs. Qing Dai, Xiaoxia Yang and Chi Li from the National Center for Nanoscience and Technology of China; Prof. Xueming Liu from Xi'an Institute of Optics and Precision Mechanics; Profs. Zhaoyu Ren and Jintao Bai from the Northwest University, Profs. Jianlin Zhao, Dong Mao, Xuetao Gan from Northwestern Polytechnical University, and their group members.

Received: ((will be filled in by the editorial staff))

Revised: ((will be filled in by the editorial staff)) Published online: ((will be filled in by the editorial staff) 
WILEY-VCH

Table 1. Typical NLO processes in 2DLMs.

\begin{tabular}{|c|c|c|c|c|c|c|c|}
\hline & Material & Emission wavelength [nm] & Fabrication & NLO coefficient $\left(\chi^{(n)}\right)^{*}$ & $\eta^{* *}$ & Thickness & Substrate \\
\hline \multirow{12}{*}{ SHG } & & $370-500^{[84]} ; 405^{[82,83,137,161]} ; 420-$ & $\operatorname{CVD}^{[83,96,97,137,161,2}$ & $0.17-1.2^{[84]} ; 2.9^{[96]} ; 500^{[83]}$ & \multirow{2}{*}{$0.18(750)^{[96]} ; 2(750)^{[95]}$} & $\mathrm{ML}^{[82-84,95-}$ & Glass $^{[82,96]} ; \mathrm{SiO}_{2}{ }^{[83,95,97,161,20}$ \\
\hline & $\mathrm{MoS}_{2}$ & $500^{[150]} ; 640-665^{[200]} ; 780^{[95-97]}$ & ${ }^{00]} ; \mathrm{ME}^{[82-84,95,150]}$ & $3200^{[82]} ; 10000^{[83]}$ & & ${ }^{97,200]} ; \mathrm{BL}^{[137,150]} ; \mathrm{FL}^{[95]}$ & ${ }^{0]} ;$ Quartz $^{[82,84]} ;$ Sapphire ${ }^{[137]}$ \\
\hline & $\mathrm{MoSe}_{2}$ & $600-900^{[90]} ; 775^{[157]}$ & $\mathrm{PLD}^{[90]} ; \mathrm{ME}^{[157]}$ & $1-5^{[90]}$ & - & $\mathrm{ML}^{[90,157]}$ & $\mathrm{SiO}_{2}{ }^{[90]} ; \mathrm{Si}^{[157]}$ \\
\hline & $\mathrm{MoS}_{2(1-x)} \mathrm{Se}_{2 x}$ & $500-900^{[142]}$ & $\mathrm{CVD}^{[142]}$ & $2-8^{[142]}$ & - & $M L^{[142]}$ & $\mathrm{SiO}_{2}{ }^{[142]}$ \\
\hline & $\mathrm{WS}_{2}$ & $415^{[87]} ; 530-660^{[152]}$ & $\mathrm{CVD}^{[87,152]}$ & $68^{[152]} ; 900^{[87]}$ & $30000(60)^{[87]}$ & $\mathrm{ML}^{[87]} ;$ Spiral $^{[152]}$ & $\mathrm{SiO}_{2}{ }^{[87]} ; \mathrm{Quartz}^{[152]}$ \\
\hline & $\mathrm{WSe}_{2}$ & $400^{[91]} ; 516-795^{[86,136,151]}$ & $\mathrm{ME}^{[86,136,151]} ; \mathrm{CVD}^{[91]}$ & $0.4-6^{[136]} ; 1000^{[91]}$ & $4(8000)^{[136]} ; 0.1(106)^{[91]}$ & $\mathrm{ML}^{[86,91,136]} ; \mathrm{BL}^{[151]}$ & $\mathrm{SiO}_{2}[86,91,136,151]$ \\
\hline & InSe & $530^{[146]}$ & $\mathrm{ME}^{[146]}$ & - & - & $9-25 \mathrm{~nm}^{[146]}$ & Quartz ${ }^{[146]}$ \\
\hline & $\mathrm{MoTe}_{2}$ & $500^{[88]}$ & $\mathrm{ME}^{[88]}$ & $15^{[88]}$ & - & $\mathrm{FL}^{[88]}$ & $\mathrm{SiO}_{2}[88]$ \\
\hline & h-BN & $405^{[82,144]}$ & $\mathrm{ME}^{[82]} ; \mathrm{CVD}^{[144]}$ & $200^{[82]}$ & - & $\mathrm{ML}^{[82]} ; \mathrm{BL}^{[144]} ; \mathrm{FL}^{[82]}$ & Quartz $^{[82]} ;$ Sapphire $^{[144]}$ \\
\hline & $\mathrm{GaSe}$ & $400^{[143]} ; 750^{[160]} ; 780^{[99]} ; 600-800^{[92]}$ & $\mathrm{ME}^{[99,143,160]} ; \mathrm{CVD}^{[92]}$ & $1.8^{[99]} ; 70-240^{[92]} ; 300^{[143]}$ & $4.8(1000)^{[99]}$ & $\mathrm{ML}^{[92]} ; 9 \mathrm{~L}^{[99,160]} ; 2-10 \mathrm{~L}^{[143]}$ & $\mathrm{SiO}_{2}{ }^{[99,143]} ; \mathrm{Quartz}^{[92]}$ \\
\hline & GaTe & $760^{[126]}$ & $\mathrm{ME}^{[126]}$ & $11.5^{[126]}$ & $1.8(290)$ & $7-57 \mathrm{~nm}^{[126]}$ & $\mathrm{SiO}_{2}^{[126]}$ \\
\hline & $\mathrm{AgInP}_{2} \mathrm{~S}_{6}$ & $400^{[147]}$ & $\mathrm{ME}^{[147]}$ & $10^{-2} \times \chi^{(2)} \operatorname{MoS} 2^{[147]}$ & - & $\mathrm{ML}^{[147]}$ & $\mathrm{SiO}_{2}^{[147]}$ \\
\hline \multirow{9}{*}{ THG } & $\mathrm{MoS}_{2}$ & $520^{[95-97]} ; 586-660^{[94]}$ & $\mathrm{CVD}^{[96,97]} ; \mathrm{ME}^{[94,95]}$ & $2.4^{[96]} ; \sim 1.2^{[94,97]}$ & $0.24(750)^{[96]} ; 32(750)^{[95]}$ & $\mathrm{ML}^{[95-97]} ; 5 \mathrm{~nm}^{[94]}$ & $\operatorname{Glass}^{[96]} ; \mathrm{SiO}_{2}{ }^{[94,95,97]}$ \\
\hline & $\mathrm{MoSe}_{2}$ & 520 & $\mathrm{ME}$ & 2.2 & $6.6(2700)$ & ML & $\mathrm{SiO}_{2}^{[203]}$ \\
\hline & $\mathrm{WS}_{2}$ & 520 & ME & 2.4 & $2.4(2700)$ & ML & $\mathrm{SiO}_{2}[203]$ \\
\hline & $\mathrm{WSe}_{2}$ & 520 & ME & 1 & $2.8(2700)$ & ML & $\mathrm{SiO}_{2}{ }^{[203]}$ \\
\hline & $\mathrm{ReS}_{2}$ & $505^{[98]}$ & $\mathrm{ME}^{[98]}$ & $53^{[98]}$ & $2(2100)^{[98]}$ & $\mathrm{ML}^{[98]}$ & Glass $^{[98]}$ \\
\hline & $\mathrm{BP}$ & $520^{[114,115,141]}$ & $\mathrm{ME}^{[114,115,141]}$ & $1.6^{[114,115]} ; 10 \times \chi^{(3)}$ graphene $^{[141]}$ & $0.7(2200)^{[114]}$ & $\sim 10 \mathrm{~nm}^{[114,115]} ; 2-4 \mathrm{~L}^{[141]}$ & $\mathrm{SiO}_{2}{ }^{[114,115]} ;$ Glass $^{[141]}$ \\
\hline & Graphene & $\begin{array}{l}263^{[47]} ; 520^{[45,96]} ; 575^{[46]} ; \\
433-550^{[70]} ; 590-1030^{[69]}\end{array}$ & $\begin{array}{l}\mathrm{CVD}^{[47,69,70,96]} \\
\mathrm{ME}^{[45,46]}\end{array}$ & $\begin{array}{c}1^{[47,96]} ; 5-10^{[70]} \\
400^{[46]} ; 42000^{[45]}\end{array}$ & $\begin{array}{c}10^{-3}(10)^{[47]} \\
0.1(1500)^{[96]} ; 1(8000)^{[45]}\end{array}$ & $\mathrm{ML}^{[45-47,69,70,96]}$ & $\begin{array}{c}\text { Glass }^{[47,96]} ; \mathrm{SiO}_{2}{ }^{[45,46,69]} ; \\
\text { Quartz }^{[70]}\end{array}$ \\
\hline & GaSe & $520^{[99]}$ & $\mathrm{ME}^{[99]}$ & $1600^{[99]}$ & $2.3(1000)^{[99]}$ & $\mathrm{FL}^{[99]}$ & $\mathrm{SiO}_{2}{ }^{[99]}$ \\
\hline & GaTe & $520^{[126]}$ & $\mathrm{ME}^{[126]}$ & $2000^{[126]}$ & $4(290)$ & $7-57 \mathrm{~nm}^{[126]}$ & $\mathrm{SiO}_{2}^{[126]}$ \\
\hline \multirow{3}{*}{ FWM } & Graphene & $\begin{array}{c}700^{[69]} ; 760-840^{[49]} ; \\
360-1566^{[70]} ; \sim 1550^{[50,51,148,177,178]}\end{array}$ & $\begin{array}{c}\operatorname{ME}^{[49,69,148]} \\
\operatorname{CVD}^{[50,51,70,177,178]}\end{array}$ & $\begin{array}{l}14000^{[49]} \\
5-300^{[70]}\end{array}$ & $\begin{array}{c}10^{-2}(80)^{[69]} \\
10^{7.3}(3.2)^{[148]} ; 10^{8}(600)^{[51]}\end{array}$ & $\begin{array}{c}\mathrm{ML}^{[49-51,69,70,177,178]} \\
\mathrm{FL}^{[148]}\end{array}$ & $\begin{array}{c}\mathrm{SiO}_{2}{ }^{[69]} ; \mathrm{Glass}^{[49]} ; \\
\text { Quartz }^{[70]} ; \mathrm{Si}^{[50,51,148,177,178]}\end{array}$ \\
\hline & $\mathrm{MoS}_{2}$ & $430-450^{[93]}$ & $\mathrm{ME}^{[93]}$ & - & - & $1-8 \mathrm{~L}^{[93]}$ & Quartz $^{[93]}$ \\
\hline & $\mathrm{BP}$ & $\sim 1550^{[116,117]}$ & $\operatorname{LPE}^{[116,117]}$ & - & $10^{3[116]} ; 10^{4[117]}$ & $4.3 \mathrm{~nm}^{[116]} ; 18 \mathrm{~nm}^{[117]}$ & Glass $^{[116,117]}$ \\
\hline
\end{tabular}

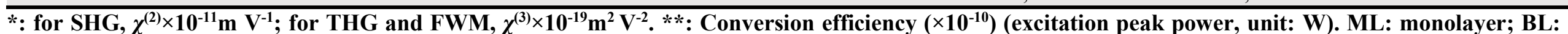

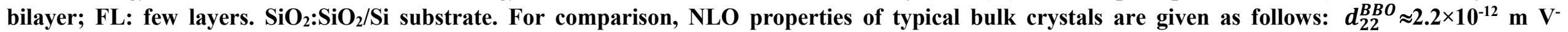
${ }^{1}{ }^{[155]} d_{33}^{\text {LiNbO }_{3}} \approx-31.8 \times 10^{-12} \mathrm{~m} \mathrm{~V}^{-1} ;^{[155]} \chi_{G a A s}^{(3)} \approx 1.4 \times 10^{-18} \mathrm{~m}^{2} \mathrm{~V}^{-2}{ }^{[2]}$ 


\section{WILEY-VCH}

(a)
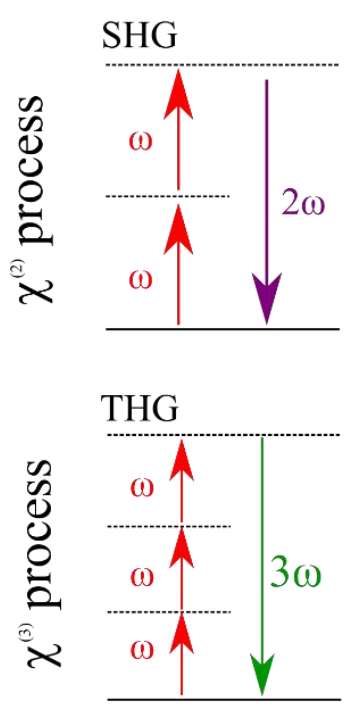
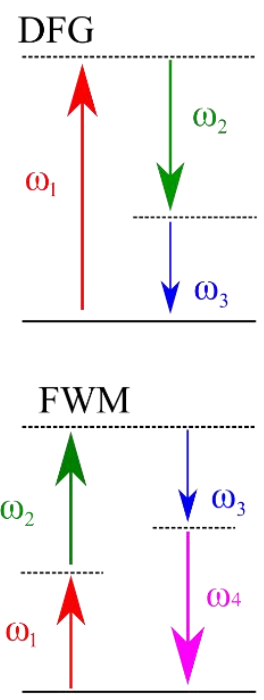

(b)

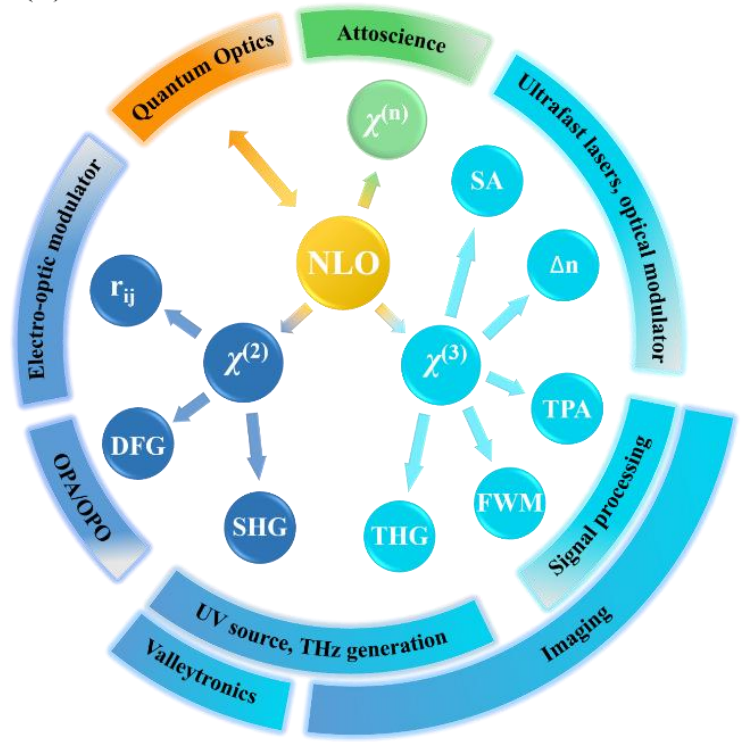

Figure 1. Fundamentals of nonlinear optics. (a) Examples of different nonlinear frequency conversion processes, such as SHG, DFG, THG, and FWM. Input photons at various frequencies (e.g., $\omega$ for SHG and THG, $\omega_{1}$ for DFG, $\omega_{1}$ and $\omega_{2}$ for FWM) can be converted into photons at different frequencies (e.g., $2 \omega$ for SHG, $3 \omega$ for THG, $\omega_{2}$ and $\omega_{3}$ for DFG, $\omega_{3}$ and $\omega_{4}$ for FWM). (b) Examples of typical NLO applications. TPA: two-photon absorption; $r_{i j}$ : electro-optic coefficient, $\Delta \mathrm{n}$ : intensity dependent refractive index change. 


\section{WILEY-VCH}

(a)

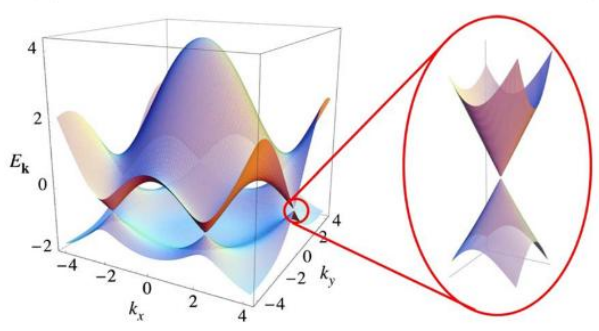

Graphene (b)

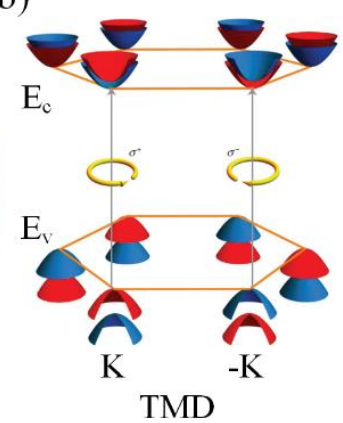

(c)

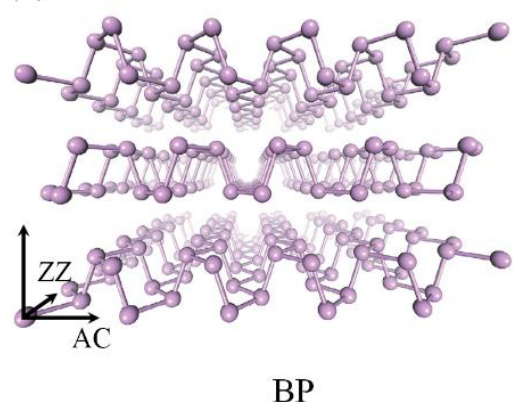

Figure 2. Fundamentals of 2DLMs. (a) Electronic band structure of graphene. Reproduced with permission. ${ }^{[22]}$ Copyright 2009, American Physical Society. (b) Electronic band structure of monolayer TMDs. Reproduced with permission. ${ }^{[264]}$ Copyright 2016, Nature Publishing Group. (c) Crystal structure of BP showing the in-plane anisotropy between armchair (AC) and zigzag (ZZ) crystal directions. Reproduced with permission. ${ }^{[264]}$ Copyright 2014, Nature Publishing Group. 
(a)

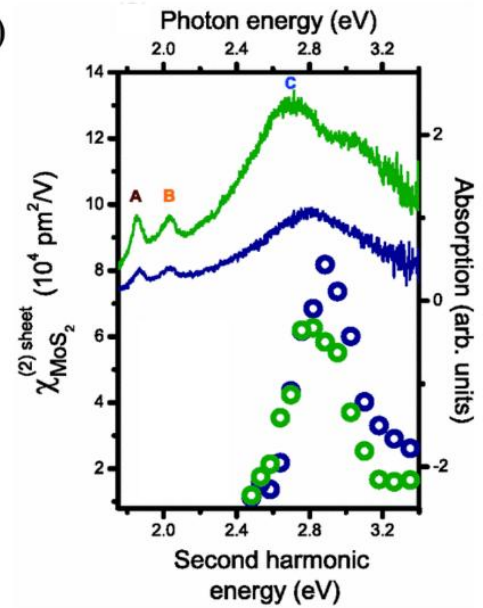

(b)

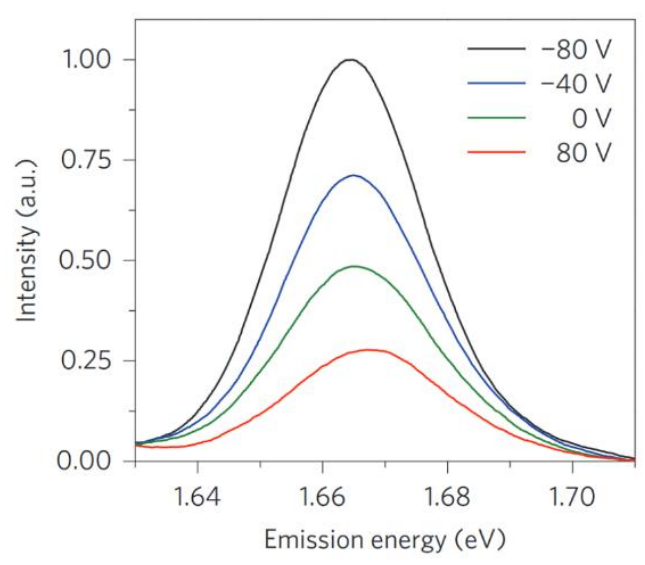

(c)

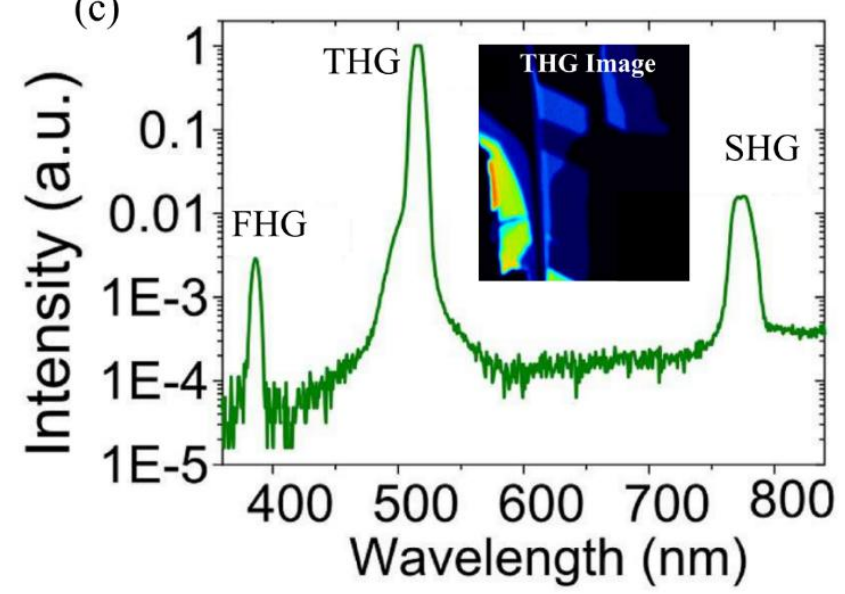

(d)

Photon energy (eV)

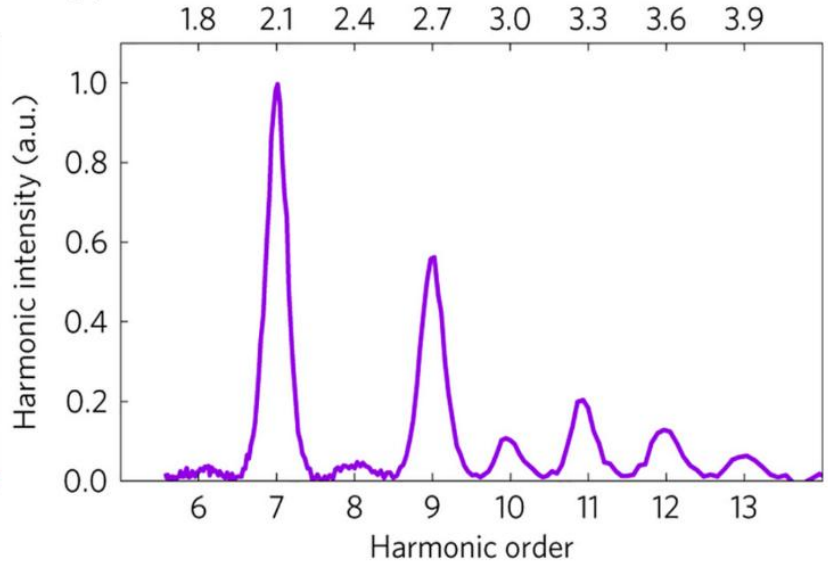

Figure 3. Harmonic generation in 2DLMs: (a) Measured absorption (solid lines) and secondorder susceptibility (circles) of $\mathrm{MoS}_{2}$. Data shown on blue is from monolayer and green is from tri-layer $\mathrm{MoS}_{2}$. Exciton peaks (i.e., A, B and C) are also indicated. Reproduced with permission. ${ }^{[22]}$ Copyright 2013, American Physical Society. (b) SHG intensity of monolayer $\mathrm{WSe}_{2}$ with different gate voltages. Reproduced with permission. ${ }^{[22]}$ Copyright 2015, Nature Publishing Group. (c) SHG, THG and FHG in monolayer $\mathrm{MoS}_{2}$. Inset: THG image of a $\mathrm{MoS}_{2}$ flake. ${ }^{[95]}$ (d) $\mathrm{HHG}$ in monolayer $\mathrm{MoS}_{2}$. Reproduced with permission. ${ }^{[22]}$ Copyright 2016, Nature Publishing Group. 

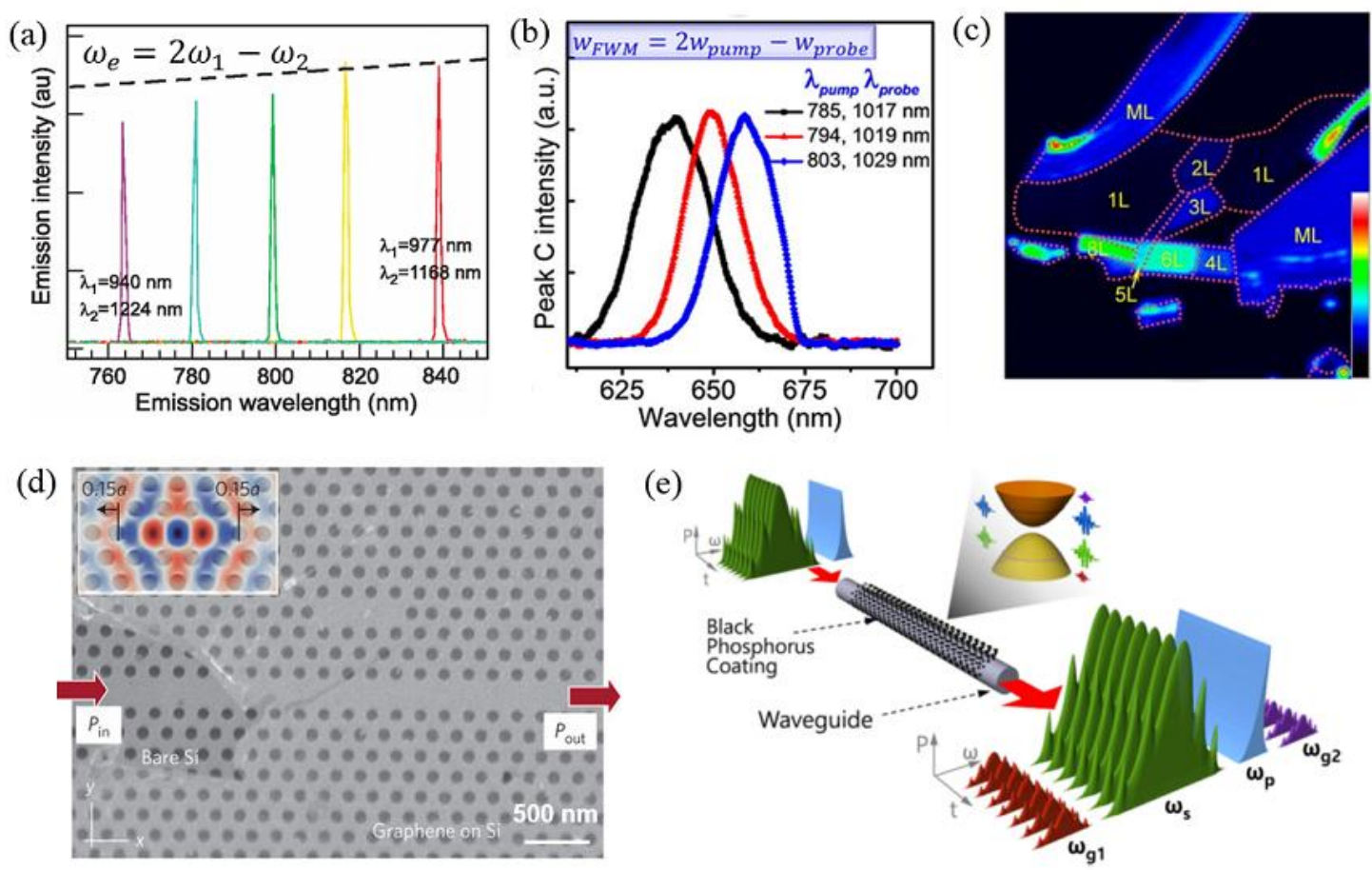

Figure 4. FWM demonstrations in 2DLMs. (a) Emission spectra of a graphene flake excited with pump pulses at different wavelengths, $\left(\lambda_{1}, \lambda_{2}\right)$. The dashed line represents the wavelength dependence predicted by nonlinear quantum response theory. Reproduced with permission. ${ }^{[22]}$ Copyright 2010, American Physical Society. (b) FWM dependence on pump-probe beams

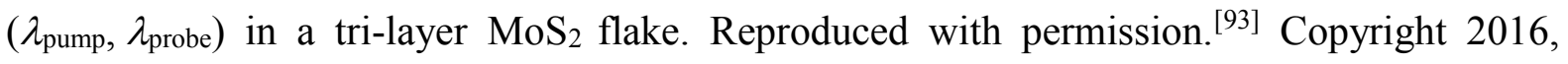
American Chemical Society. (c) FWM images of a mechanically exfoliated few-layer $\mathrm{MoS}_{2}$ flake with different thicknesses. Reproduced with permission. ${ }^{[93]}$ Copyright 2016, American Chemical Society. (d) Graphene integrated photonic crystal cavity for FWM. Inset: $\mathbf{E}_{\mathrm{z}}$-field distribution from computation. Reproduced with permission. ${ }^{[93]}$ Copyright 2012, Nature Publishing Group. (e) Schematic illustration of FWM-based wavelength conversion in a BPdeposited integrated fiber device. Inset: bandgap of BP. Reproduced under the terms of the CCBY license. ${ }^{[16]}$ Copyright 2017, Nature Publishing Group. 
(a)

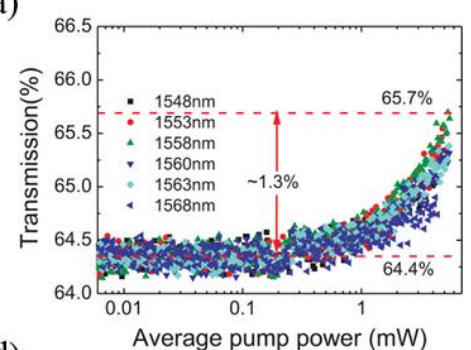

(d)

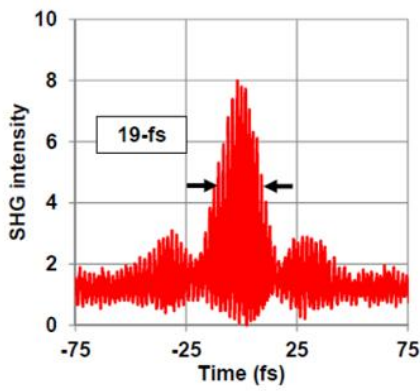

(b)

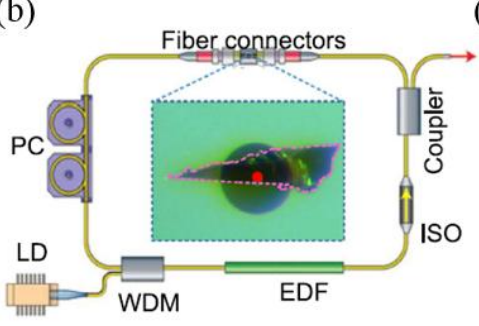

(e)

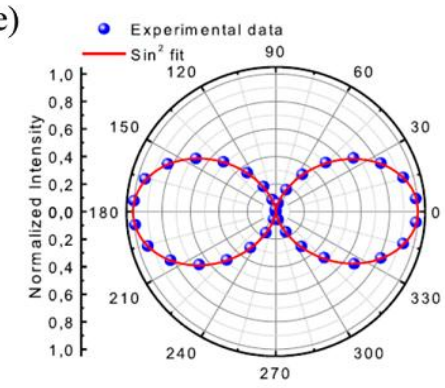

(c)

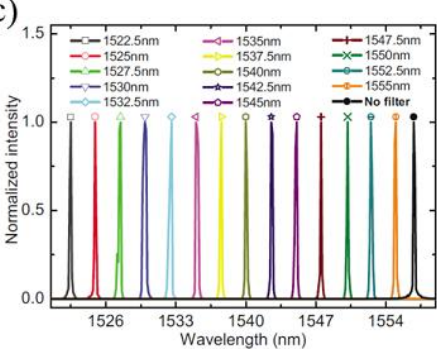

(f)

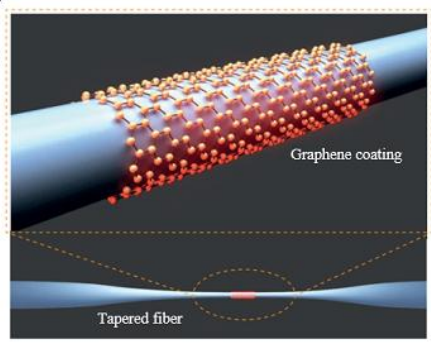

Figure 5. Saturable absorption in 2DLMs. (a) NLO absorption of graphene at different wavelengths. (b) A typical configuration of 2DLMs based ultrafast fibre lasers. Inset: an optical image of a BP (marked by pink dotted line) integrated fiber device. The red dot indicates the optical fibre core. PC: polarization controller; LD: laser diode; WDM: wavelength division multiplexer; EDF: Erbium-doped fiber; ISO: isolator. (c) Broadband tunable output spectra of a graphene Q-switched fiber laser. ${ }^{[189]}$ (d) Sub-20 fs optical pulses generated with a graphene mode-locked laser. Reproduced with permission. ${ }^{[192]}$ Copyright 2017, Optical Society of America. (e) Linearly polarized output of a BP mode-locked fiber laser. (f) Schematic of a graphene-coated microfiber ultrafast all-optical modulator. Image courtesy of Prof. Liming Tong. 


\section{WILEY-VCH}

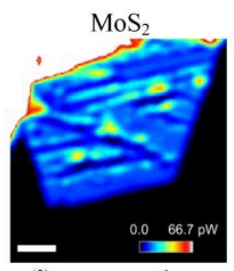

$\chi^{(2)} \approx 5.5 \mathrm{pm} \mathrm{V}^{-1}$ $\chi^{(3)} \approx 3.6 \times 10^{-19} \mathrm{~m}^{2} \mathrm{~V}^{-2}$

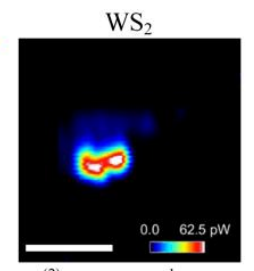

$\chi^{(2)} \approx 17 \mathrm{pm} \mathrm{V}^{-1}$
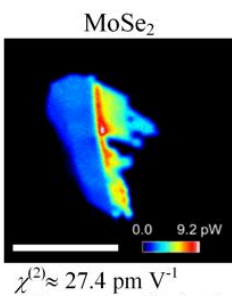

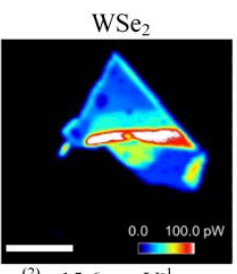

$\chi^{(2)} \approx 15.6 \mathrm{pm} \mathrm{V}^{-1}$

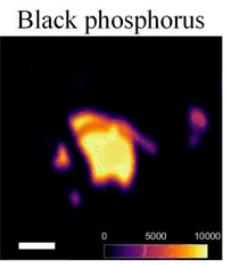

$\chi^{(3)} \approx 1.6 \times 10^{-19} \mathrm{~m}^{2} \mathrm{~V}^{-2}$
Graphene

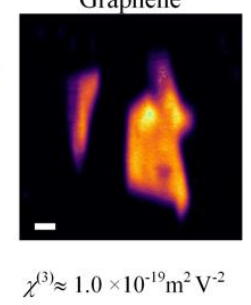

Figure 6. THG imaging of a few common 2DLMs (i.e., mono- and few-layer TMDs, BP, and graphene). The typical $\chi^{(2)}$ and $\chi^{(3)}$ of monolayers are listed. Note that $\chi^{(2)}$ of BP and graphene is zero due to their centrosymmetric structures. The pump wavelength: $1560 \mathrm{~nm}$. Scale bar: 10 $\mu \mathrm{m}$. 


\section{WILEY-VCH}
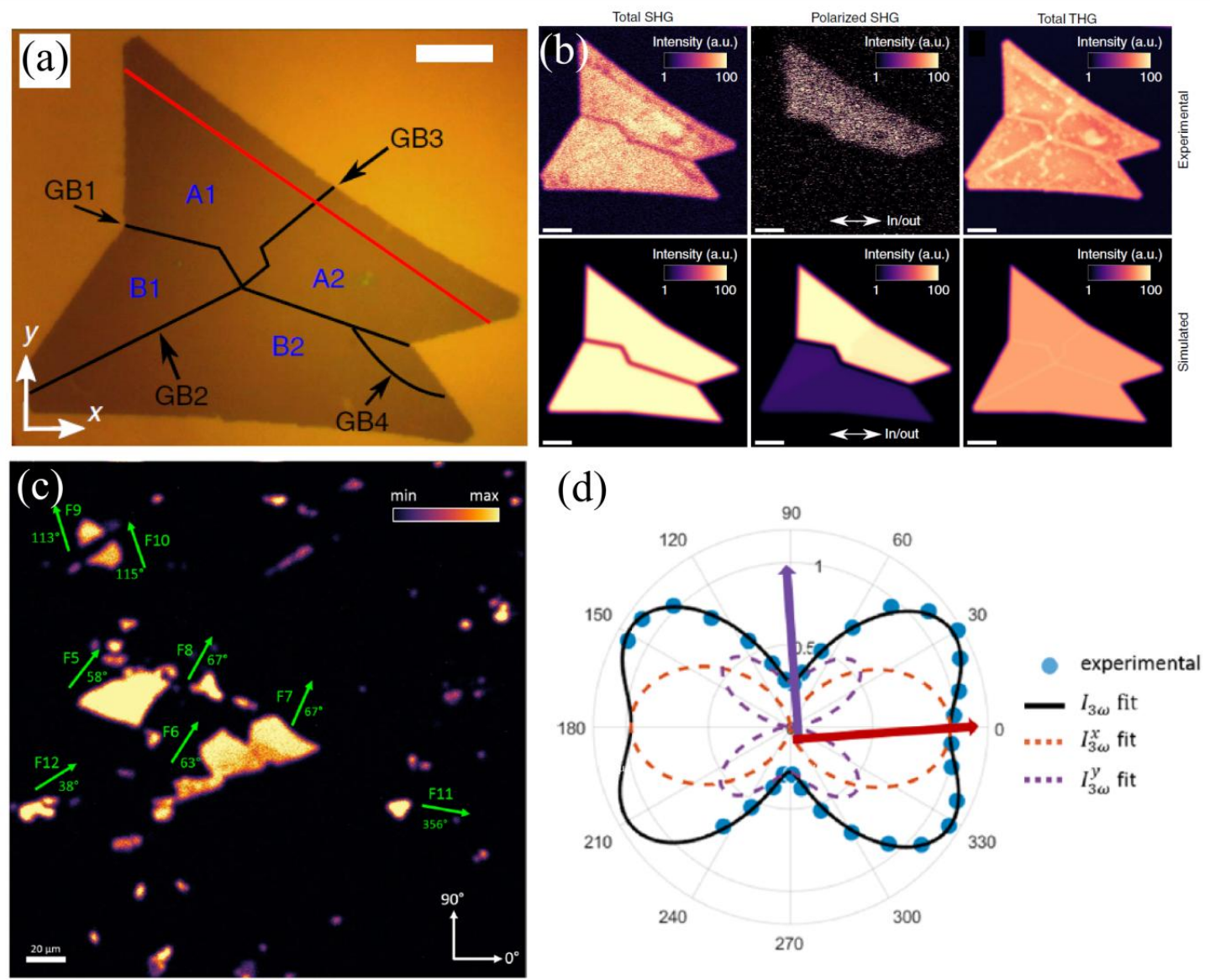

Figure 7. Nonlinear optics for 2D material characterization. (a) Optical image of a CVD grown $\mathrm{MoS}_{2}$ flake highlighting the different grain boundaries (GBs 1-4). (b) Multiphoton microscopy images of the CVD grown $\mathrm{MoS}_{2}$ flake show how different crystal domains and GBs can be resolved from multiphoton microscopy images by varying the excitation laser polarization direction. The experimentally measured contrast (upper panel) agrees extremely well with the simulated multiphoton signal (lower panel). Scale bars: $10 \mu \mathrm{m}$. (c) THG image with overlaid AC crystal directions of 8 different BP flakes which crystal directions were characterized utilizing the angular dependence of THG to the excitation light polarization direction. (d) Polar plot shows the measured angular dependence of the THG and demonstrates that the THG intensity changes with polarization directions. The red and purple arrows indicate the AC and $\mathrm{ZZ}$ crystal directions of the measured BP flake. 

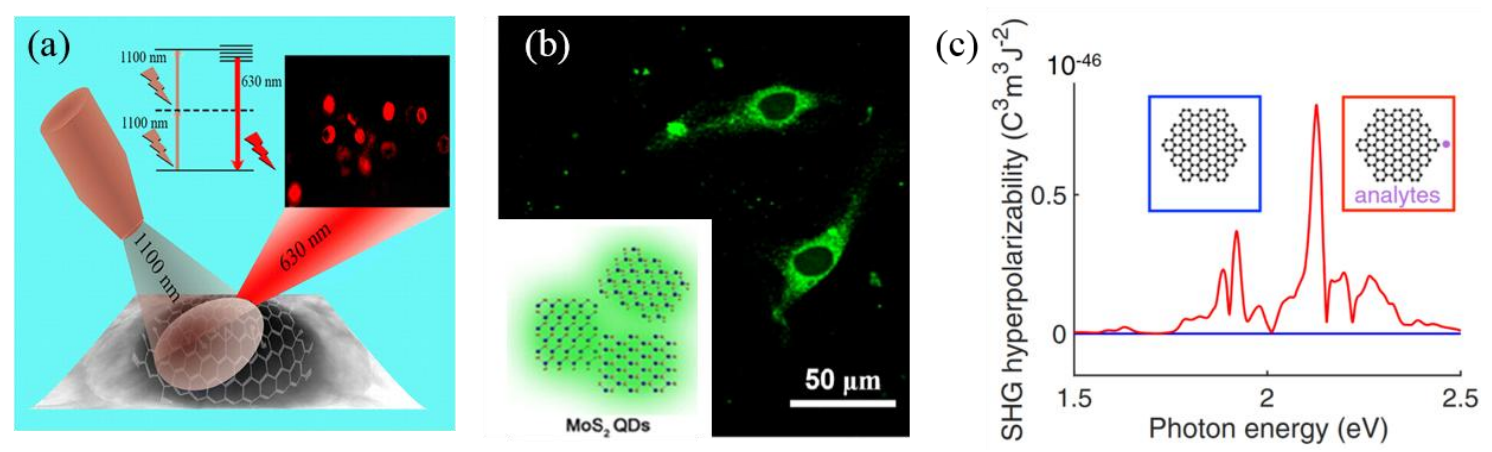

Figure 8. Nonlinear imaging and sensing with 2DLMs. (a) Schematic setup of aptamer-bound graphene oxide-based two-photon luminescence imaging platform for selective cancer cell imaging. Reproduced with permission. ${ }^{[210]}$ Copyright 2014, American Chemical Society. (b) Up-conversion cellular imaging of $\mathrm{MoS}_{2}$ quantum dots (inset) under an $800 \mathrm{~nm}$ excitation. Reproduced with permission. ${ }^{[211]}$ Copyright 2016, American Chemical Society. (c) Secondharmonic polarizability of the graphene nano-hexagon in the absence (blue curves) or presence (red curves) of a nearby charge-carrying molecule. Reproduced with permission. ${ }^{[218]}$ Copyright 2016, American Physical Society. 


\section{WILEY-VCH}

(a)

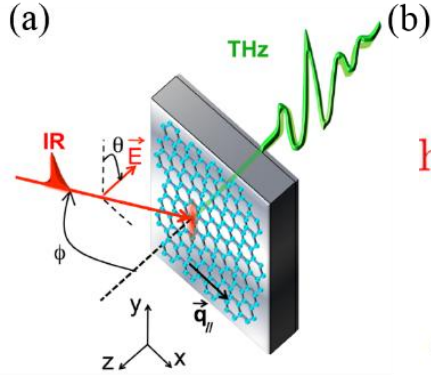

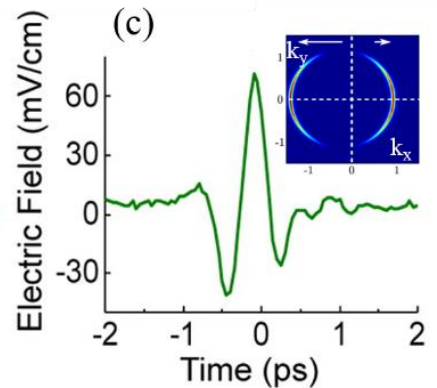

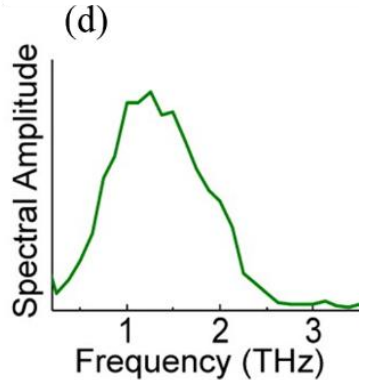

Figure 9. THz generation in graphene. (a) A femtosecond optical pump pulse illuminates the multilayer graphene and transient photon drag current generated in the plane of the graphene sheets emits a THz pulse. (b) Transient non-thermal electron and hole distributions in the interband regime for an oblique illumination in graphene. (c) Experimental electric field waveform emitted by the multilayer graphene illuminated by s-polarized femtosecond optical pulses at $800 \mathrm{~nm}$ central wavelength under an incidence angle $\phi=25^{\circ}$ and its associated spectrum (d). Inset of Figure (c): Nonequilibrium electron population distribution generated by p-polarized femtosecond optical pulses at oblique incidence. Reproduced with permission. ${ }^{[226]}$, Copyright 2014, American Chemical Society. 
(a)

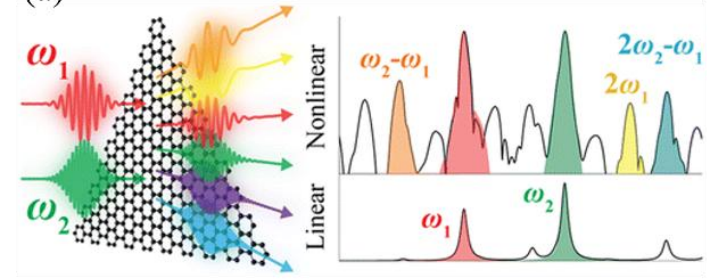

(b)

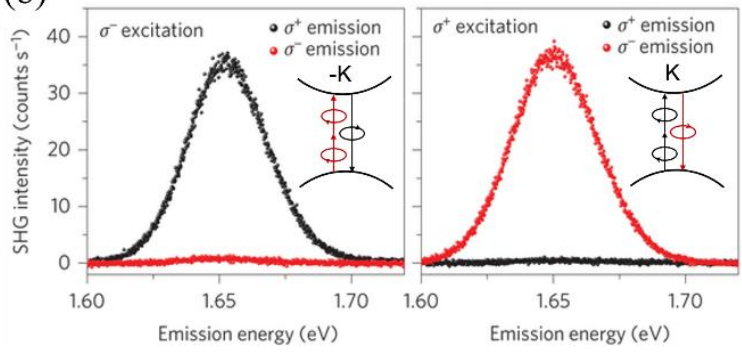

Figure 10. Nonlinear optics with polaritons in 2DLMs. (a) Illustration of an armchair triangular graphene nano-island; enhanced wave-mixing when one or more of the input or output frequencies coincide with a plasmon resonance of graphene nanoislands. Reproduced with permission. ${ }^{[226]}$, Copyright 2015, American Chemical Society. (b) Valley-dependent SHG selection rules: circular polarization-resolved SHG spectra showing the generation of countercircular SHG. Inset: Inter-band valley optical selection rules for SHG. Reproduced with permission. ${ }^{[226]}$, Copyright 2015, Nature Publishing Group. 


\section{WILEY-VCH}

(a)

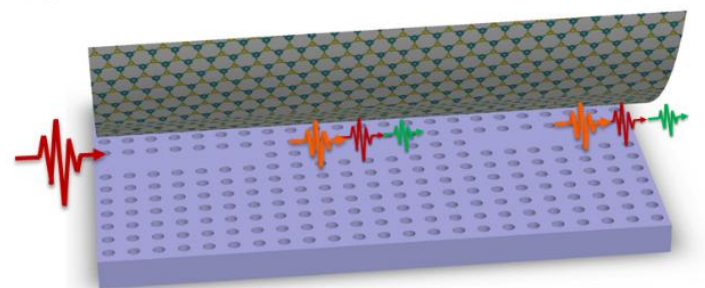

(c)

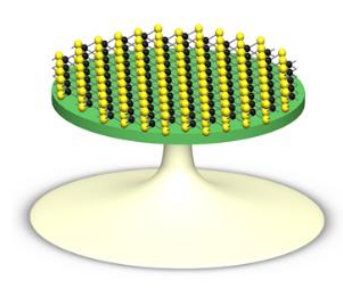

(d)

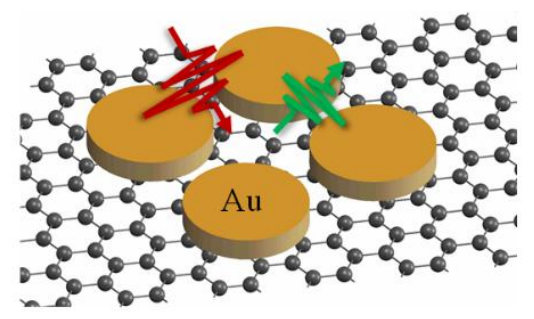

(b)

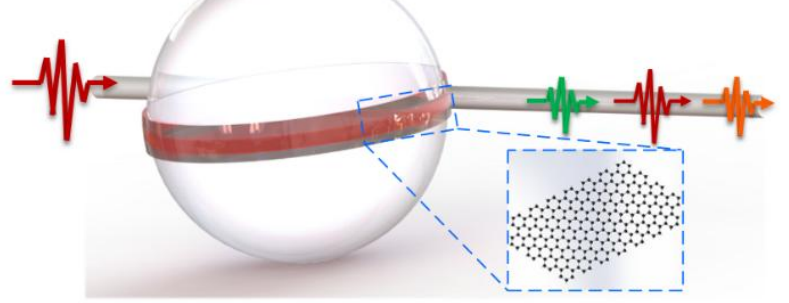

(e)

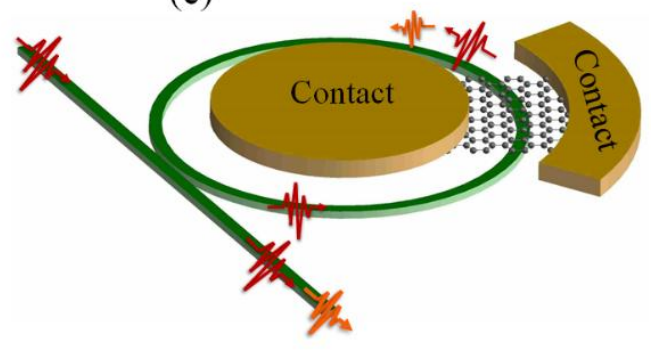

Figure 11. Optical nonlinearity enhancement in 2DLMs by integrating with (a) photonic crystal cavity, (b) microsphere cavity (inset: coated 2DLMs), (c) microdisk resonator, (d) metallic plasmonic structures. (e) Electrically tunable NLO devices based on 2DLMs. 


\section{References:}

[1] Y. R. Shen, The Principles of Nonlinear Optics, Wiley Press, New York 1984.

[2] R. W. Boyd, Nonlinear Optics, Academic Press, New York 2007.

[3] G. P. Agrawal, Applications of Nonlinear Fiber Optics, Academic Press, London 2001;

B. E. A. Saleh, M. C. Teich, Fundamentals of Photonics, Wiley, Hoboken, New Jersey 2007.

[4] E. Garmire, Opt. Express 2013, 21, 30532.

[5] U. Keller, Nature 2003, 424, 831.

[6] C. Li, Nonlinear Optics: Principles and Applications, Springer, Singapore 2017.

[7] E. L. Wooten, K. M. Kissa, A. Yi-Yan, E. J. Murphy, D. A. Lafaw, P. F. Hallemeier, D. Maack, D. V. Attanasio, D. J. Fritz, Gregory J. McBrien, D. E. Bossi, IEEE J. Sel. Top. Quantum Electron. 2000, 6, 69.

[8] B. Nabet, Photodetectors: Materials, Devices and Applications, Elsevier, London 2015.

[9] M. Duelli, G. Montemezzani, M. Zgonik, P. Günter, in Nonlinear Optical Effects and Materials, Springer, 2000, 375.

[10]Z. P. Sun, R. N. Li, B. Yong, X. D. Yang, Z. Ying, G. L. Wang, W. L. Zhao, H. B. Zhang, H. Wei, D. F. Cui, Z. Y. Xu, Opt. Commun. 2004, 241, 167; G. K. Samanta, G. R. Fayaz, Z. Sun, M. Ebrahim-Zadeh, Opt. Lett. 2007, 32, 400; Z. P. Sun, R. N. Li, Y. Bi, X. D. Yang, Y. Bo, W. Hou, X. C. Lin, H. B. Zhang, D. F. Cui, Z. Y. Xu, Opt. Express 2004, 12, 6428; Z. Sun, M. Ghotbi, M. Ebrahim-Zadeh, Opt. Express 2007, 15, 4139; Y. Bo, A. C.

Geng, Y. Bi, Z. P. Sun, X. D. Yang, Q. J. Peng, H. Q. Li, R. N. Li, D. F. Cui, Z. Y. Xu, Appl. Opt. 2006, 43, 2499.

[11]W. R. Zipfel, R. M. Williams, W. W. Webb, Nat. Biotechno. 2003, 21, 1369.

[12]Z. Sun, A. C. Ferrari, Nat. Photonics 2011, 5, 446.

[13]K. J. Vahala, Nature 2003, 424, 839.

[14]J. Leuthold, C. Koos, W. Freude, Nat. Photonics 2010, 4, 535.

[15]D. E. Chang, V. Vuletic, M. D. Lukin, Nat. Photonics 2014, 8, 685; S. L. Rolston, W. D. Phillips, Nature 2002, 416, 219.

[16] M. Kauranen, A. V. Zayats, Nat. Photonics 2012, 6, 737; C. M. Soukoulis, M. Wegener, Nat. Photonics 2011, 5, 523.

[17]J. Witzens, T. Baehr-Jones, M. Hochberg, Nat. Photonics 2010, 4, 10.

[18] J. L. O'Brien, A. Furusawa, J. Vučković, Nat. Photonics 2009, 3, 687.

[19]A. C. Ferrari, F. Bonaccorso, V. Fal'ko, K. S. Novoselov, S. Roche, P. Boggild, S.

Borini, F. H. Koppens, V. Palermo, N. Pugno, J. A. Garrido, R. Sordan, A. Bianco, L.

Ballerini, M. Prato, E. Lidorikis, J. Kivioja, C. Marinelli, T. Ryhanen, A. Morpurgo, J. N.

Coleman, V. Nicolosi, L. Colombo, A. Fert, M. Garcia-Hernandez, A. Bachtold, G. F.

Schneider, F. Guinea, C. Dekker, M. Barbone, Z. Sun, C. Galiotis, A. N. Grigorenko, G.

Konstantatos, A. Kis, M. Katsnelson, L. Vandersypen, A. Loiseau, V. Morandi, D. Neumaier, E. Treossi, V. Pellegrini, M. Polini, A. Tredicucci, G. M. Williams, B. H. Hong, J. H. Ahn, J. M. Kim, H. Zirath, B. J. van Wees, H. van der Zant, L. Occhipinti, A. Di Matteo, I. A.

Kinloch, T. Seyller, E. Quesnel, X. Feng, K. Teo, N. Rupesinghe, P. Hakonen, S. R. Neil, Q. Tannock, T. Lofwander, J. Kinaret, Nanoscale 2015, 7, 4598.

[20] K. S. Novoselov, A. K. Geim, S. V. Morozov, D. Jiang, Y. Zhang, S. V. Dubonos, I. V. Grigorieva, A. A. Firsov, Science 2004, 306, 666.

[21]A. K. Geim, K. S. Novoselov, Nat. Mater. 2007, 6, 183; K. S. Novoselov, V. I. Fal'ko, L. Colombo, P. R. Gellert, M. G. Schwab, K. Kim, Nature 2012, 490, 192.

[22] A. H. Castro Neto, F. Guinea, N. M. R. Peres, K. S. Novoselov, A. K. Geim, Rev. Mod. Phys. 2009, 81, 109. 
[23]Z. Sun, T. Hasan, F. Torrisi, D. Popa, G. Privitera, F. Wang, F. Bonaccorso, D. M. Basko, A. C. Ferrari, ACS Nano 2010, 4, 803.

[24]Q. Bao, H. Zhang, Y. Wang, Z. Ni, Y. Yan, Z. X. Shen, K. P. Loh, D. Y. Tang, Adv. Funct. Mater. 2009, 19, 3077.

[25] T. Hasan, Z. Sun, F. Wang, F. Bonaccorso, P. H. Tan, A. G. Rozhin, A. C. Ferrari, Adv. Mater. 2009, 21, 3874.

[26]F. Bonaccorso, Z. Sun, T. Hasan, A. C. Ferrari, Nat. Photonics 2010, 4, 611.

[27] M. Liu, X. Yin, E. Ulin-Avila, B. Geng, T. Zentgraf, L. Ju, F. Wang, X. Zhang, Nature 2011, 474, 64; F. Ceballos, H. Zhao, Adv. Funct. Mater. 2016, 27, 1604509.

[28] W. Li, B. G. Chen, C. Meng, W. Fang, Y. Xiao, X. Y. Li, Z. F. Hu, Y. X. Xu, L. M.

Tong, H. Q. Wang, W. T. Liu, J. M. Bao, Y. R. Shen, Nano Lett. 2014, 14, 955.

[29]Z. Sun, T. Hasan, A. C. Ferrari, Physica E 2012, 44, 1082.

[30]A. Martinez, Z. Sun, Nat. Photonics 2013, 7, 842.

[31]Z. Sun, A. Martinez, F. Wang, Nat. Photonics 2016, 10, 227.

[32]X. Liu, Q. Guo, J. Qiu, Adv. Mater. 2017, 29, 1605886; S. Yu, X. Wu, Y. Wang, X. Guo, L. Tong, Adv. Mater. 2017, 29, 1606128.

[33] T. Low, A. Chaves, J. D. Caldwell, A. Kumar, N. X. Fang, P. Avouris, T. F. Heinz, F.

Guinea, L. Martin-Moreno, F. Koppens, Nat. Mater. 2017, 16, 182.

[34]K. F. Mak, J. Shan, Nat. Photonics 2016, 10, 216.

[35]F. N. Xia, H. Wang, D. Xiao, M. Dubey, A. Ramasubramaniam, Nat. Photonics 2014, 8, 899; F. H. Koppens, T. Mueller, P. Avouris, A. C. Ferrari, M. S. Vitiello, M. Polini, Nat.

Nanotechnol. 2014, 9, 780; S. Manzeli, D. Ovchinnikov, D. Pasquier, O. V. Yazyev, A. Kis, Nat. Rev. Mater. 2017, 2, 17033.

[36]Q. H. Wang, K. Kalantar-Zadeh, A. Kis, J. N. Coleman, M. S. Strano, Nat. Nanotechnol. 2012, 7, 699 .

[37] M. Ghotbi, Z. Sun, A. Majchrowski, E. Michalski, I. V. Kityk, M. Ebrahim-Zadeh, Appl. Phys. Lett. 2006, 89, 173124.

[38] X. C. Lin, Y. P. Kong, Y. Zhang, J. Zhang, A. Y. Yao, Y. Bi, Z. P. Sun, D. F. Cui, R. N. Li, L. A. Wu, Z. Y. Xu, Chin. Phys. 2004, 13, 1042.

[39] J. Sipe, D. Moss, H. van Driel, Phys. Rev. B 1987, 35, 1129.

[40] T. F. Heinz, in Nonlinear surface electromagnetic phenomena, Elsevier Science, 1991.

[41] A. C. Ferrari, D. M. Basko, Nat. Nanotechnol. 2013, 8, 235; X. Zhang, Q. H. Tan, J. B.

Wu, W. Shi, P. H. Tan, Nanoscale 2016, 8, 6435; X. Zhang, X.-F. Qiao, W. Shi, J.-B. Wu,

D.-S. Jiang, P.-H. Tan, Chem. Soc. Rev. 2015, 44, 2757.

[42]R. R. Nair, P. Blake, A. N. Grigorenko, K. S. Novoselov, T. J. Booth, T. Stauber, N. M.

R. Peres, A. K. Geim, Science 2008, 320, 1308; K. F. Mak, M. Y. Sfeir, Y. Wu, C. H. Lui, J.

A. Misewich, T. F. Heinz, Phys. Rev. Lett. 2008, 101, 196405; A. B. Kuzmenko, E. van

Heumen, F. Carbone, D. van der Marel, Phys. Rev. Lett. 2008, 100, 117401.

[43]Z. P. Sun, D. Popa, T. Hasan, F. Torrisi, F. Q. Wang, E. J. R. Kelleher, J. C. Travers, V. Nicolosi, A. C. Ferrari, Nano Res. 2010, 3, 653.

[44]F. Bo, H. Yi, X. Xiaosheng, Z. Hongwei, S. Zhipei, Y. Changxi, IEEE J. Sel. Top.

Quantum Electron. 2014, 20, 411.

[45] A. Säynätjoki, L. Karvonen, J. Riikonen, W. Kim, S. Mehravar, R. A. Norwood, N.

Peyghambarian, H. Lipsanen, K. Kieu, ACS Nano 2013, 7, 8441.

[46] N. Kumar, J. Kumar, C. Gerstenkorn, R. Wang, H.-Y. Chiu, A. L. Smirl, H. Zhao, Phys.

Rev. B 2013, 87, 121406.

[47] S.-Y. Hong, J. I. Dadap, N. Petrone, P.-C. Yeh, J. Hone, R. M. Osgood, Phys. Rev. X 2013, 3, 021014. 
[48]H. Nasari, M. S. Abrishamian, RSC Adv. 2016, 6, 50190.

[49]E. Hendry, P. J. Hale, J. Moger, A. K. Savchenko, S. A. Mikhailov, Phys. Rev. Lett. 2010, 105, 097401.

[50]T. Gu, N. Petrone, J. F. McMillan, A. v. d. Zande, M. Yu, G. Q. Lo, D. L. Kwong, J. Hone, C. W. Wong, Nat. Photonics 2012, 6, 554.

[51] Y. Wu, B. Yao, Q. Feng, X. Cao, X. Zhou, Y. Rao, Y. Gong, W. Zhang, Z. Wang, Y. Chen, Photonics Res. 2015, 3, A64.

[52]I. Al-Naib, M. Poschmann, M. M. Dignam, Phys. Rev. B 2015, 91, 205407.

[53]N. Vermeulen, D. Castelló-Lurbe, J. Cheng, I. Pasternak, A. Krajewska, T. Ciuk, W. Strupinski, H. Thienpont, J. Van Erps, Phys. Rev. Applied 2016, 6, 044006; R. Wu, Y. Zhang, S. Yan, F. Bian, W. Wang, X. Bai, X. Lu, J. Zhao, E. Wang, Nano Lett. 2011, 11, 5159.

[54]N. Yoshikawa, T. Tamaya, K. Tanaka, Science 2017, 356, 736.

[55]D. Sun, C. Divin, J. Rioux, J. E. Sipe, C. Berger, W. A. de Heer, P. N. First, T. B. Norris, Nano Lett. 2010, 10, 1293.

[56] J. Wang, Y. Hernandez, M. Lotya, J. N. Coleman, W. J. Blau, Adv. Mater. 2009, 21, 2430.

[57]H. Zhang, S. Virally, Q. Bao, N. Godbout, L. K. Ping, a. P. Kockaert, Opt. Lett. 2012, 37, 1856; H. Yang, X. Feng, Q. Wang, H. Huang, W. Chen, A. T. S. Wee, W. Ji, Nano Lett. 2011, 11, 2622; E. Dremetsika, B. Dlubak, S. P. Gorza, C. Ciret, M. B. Martin, S. Hofmann, P. Seneor, D. Dolfi, S. Massar, P. Emplit, P. Kockaert, Opt. Lett. 2016, 41, 3281.

[58] S. Yu, X. Wu, K. Chen, B. Chen, X. Guo, D. Dai, L. Tong, W. Liu, Y. Ron Shen, Optica 2016, 3, 541.

[59]D. Sun, Z. K. Wu, C. Divin, X. B. Li, C. Berger, W. A. de Heer, P. N. First, T. B. Norris, Phys. Rev. Lett. 2008, 101, 157402.

[60]M. Breusing, C. Ropers, T. Elsaesser, Phys. Rev. Lett. 2009, 102, 086809.

[61] J. J. Dean, H. M. van Driel, Appl. Phys. Lett. 2009, 95, 261910.

[62] J. J. Dean, H. M. van Driel, Phys. Rev. B 2010, 82, 125411.

[63] M. T. Manzoni, I. Silveiro, F. J. G. d. Abajo, D. E. Chang, New J. Phys. 2015, 17, 083031 .

[64]B. Majerus, J. Butet, G. D. Bernasconi, R. T. Valapu, M. Lobet, L. Henrard, O. J. F. Martin, Opt. Express 2017, 25, 27015.

[65]Y. Q. An, F. Nelson, J. U. Lee, A. Diebold, Nano Lett. 2013, 13, 2104.

[66] S. J. Brun, T. G. Pedersen, Phys. Rev. B 2015, 91, 205405.

[67] S. Wu, L. Mao, A. M. Jones, W. Yao, C. Zhang, X. Xu, Nano Lett. 2012, 12, 2032.

[68]F. Bonaccorso, A. Lombardo, T. Hasan, Z. Sun, L. Colombo, A. C. Ferrari, Mater. Today 2012, 15, 564.

[69] G. Soavi, G. Wang, H. Rostami, D. Purdie, D. D. Fazio, T. Ma, B. Luo, J. Wang, A. K. Ott, D. Yoon, S. Bourelle, J. E. Muench, I. Goykhman, S. D. Conte, M. Celebrano, A. Tomadin, M. Polini, G. Cerullo, A. C. Ferrari, arXiv 2017, 1710.03694.

[70]T. Jiang, D. Huang, J. Cheng, X. Fan, Z. Zhang, Y. Shan, Y. Yi, Y. Dai, L. Shi, K. Liu, C. Zeng, J. Zi, J. E. Sipe, Y.-R. Shen, W.-T. Liu, S. Wu, arXiv 2017, 1710.04758.

[71] C.-C. Lee, J. M. Miller, T. R. Schibli, Appl. Phys. B 2012, 108, 129.

[72]J. L. Cheng, N. Vermeulen, J. E. Sipe, Phys. Rev. B 2015, 91, 235320; S. A. Mikhailov, Phys. Rev. B 2016, 93, 085403; K. Alexander, N. A. Savostianova, S. A. Mikhailov, B. Kuyken, D. Van Thourhout, ACS Photon. 2017, DOI:10.1021/acsphotonics.7b00559.

[73] J. D. Cox, F. Javier Garcia de Abajo, Nat. Commun. 2014, 5, 5725. 
[74]D. Li, H. Xue, M. Qi, Y. D. Wang, S. Aksimsek, N. Chekurov, W. Kim, C. F. Li, J. Riikonen, F. W. Ye, Q. Dai, Z. Y. Ren, J. T. Bai, T. Hasan, H. Lipsanen, Z. P. Sun, 2D Mater. 2017, 4, 025095.

[75]Z. G. Yu, Y. Cai, Y. W. Zhang, Sci. Rep. 2015, 5, 13783.

[76] J. S. Ross, P. Klement, A. M. Jones, N. J. Ghimire, J. Yan, D. G. Mandrus, T. Taniguchi, K. Watanabe, K. Kitamura, W. Yao, D. H. Cobden, X. Xu, Nat. Nanotechnol. 2014, 9, 268; B. W. H. Baugher, H. O. H. Churchill, Y. Yang, P. Jarillo-Herrero, Nat. Nanotechnol. 2014, 9, 262; A. Pospischil, M. M. Furchi, T. Mueller, Nat. Nanotechnol. 2014, 9, 257; F. Withers, O. Del Pozo-Zamudio, A. Mishchenko, A. P. Rooney, A. Gholinia, K. Watanabe, T.

Taniguchi, S. J. Haigh, A. K. Geim, A. I. Tartakovskii, K. S. Novoselov, Nat. Mater. 2015, 14, 301; Y.-Q. Bie, G. Grosso, M. Heuck, M. M. Furchi, Y. Cao, J. Zheng, D. Bunandar, E. Navarro-Moratalla, L. Zhou, D. K. Efetov, T. Taniguchi, K. Watanabe, J. Kong, D. Englund, P. Jarillo-Herrero, Nat. Nanotechnol. 2017, doi:10.1038/nnano.2017.209.

[77] A. Kumar, P. K. Ahluwalia, Eur. Phys. J. B 2012, 85, 186.

[78] A. Splendiani, L. Sun, Y. Zhang, T. Li, J. Kim, C. Y. Chim, G. Galli, F. Wang, Nano Lett. 2010, 10, 1271; K. F. Mak, C. Lee, J. Hone, J. Shan, T. F. Heinz, Phys. Rev. Lett. 2010, $105,136805$.

[79] K. F. Mak, K. He, C. Lee, G. H. Lee, J. Hone, T. F. Heinz, J. Shan, Nat. Mater. 2013, 12, 207; Y. Lin, X. Ling, L. Yu, S. Huang, A. L. Hsu, Y.-H. Lee, J. Kong, M. S. Dresselhaus, T. Palacios, Nano Lett. 2014, 14, 5569; G. Plechinger, P. Nagler, J. Kraus, N. Paradiso, C.

Strunk, C. Schüller, T. Korn, Phys. Status Solidi RRL 2015, 9, 457; J. Yang, T. Lu, Y. W. Myint, J. Pei, D. Macdonald, J.-C. Zheng, Y. Lu, ACS Nano 2015, 9, 6603.

[80] K. P. Wang, J. Wang, J. T. Fan, M. Lotya, A. O'Neill, D. Fox, Y. Y. Feng, X. Y. Zhang, B. X. Jiang, Q. Z. Zhao, H. Z. Zhang, J. N. Coleman, L. Zhang, W. J. Blau, ACS Nano 2013, 7, 9260 .

[81]F. Bonaccorso, Z. Sun, Opt. Mater. Express 2013, 4, 63.

[82] Y. Li, Y. Rao, K. Mak, Y. You, S. Wang, C. Dean, T. F. Heinz, Nano Lett. 2013, 13, 3329.

[83]N. Kumar, S. Najmaei, Q. Cui, F. Ceballos, P. M. Ajayan, J. Lou, H. Zhao, Phys. Rev. B 2013, 87, 161403.

[84] L. Malard, T. Alencar, A. P. M. Barboza, K. F. Mak, A. de Paula, Phys. Rev. B 2013, 87, 201401.

[85]H. Zeng, G.-B. Liu, J. Dai, Y. Yan, B. Zhu, R. He, L. Xie, S. Xu, X. Chen, W. Yao, X. Cui, Sci. Rep. 2013, 3, 1608.

[86] G. Wang, X. Marie, I. Gerber, T. Amand, D. Lagarde, L. Bouet, M. Vidal, A. Balocchi, B. Urbaszek, Phys. Rev. Lett. 2015, 114, 097403.

[87] C. Janisch, Y. Wang, D. Ma, N. Mehta, A. L. Elias, N. Perea-Lopez, M. Terrones, V. Crespi, Z. Liu, Sci. Rep. 2014, 4, 5530.

[88] R. Beams, L. G. Cancado, S. Krylyuk, I. Kalish, B. Kalanyan, A. K. Singh, K. Choudhary, A. Bruma, P. M. Vora, F. Tavazza, A. V. Davydov, S. J. Stranick, ACS Nano 2016, 10, 9626.

[89] C. Janisch, N. Mehta, D. Ma, A. L. Elías, N. Perea-López, M. Terrones, Z. Liu, Opt. Lett. 2014, 39, 383.

[90] C. T. Le, D. J. Clark, F. Ullah, V. Senthilkumar, J. I. Jang, Y. Sim, M.-J. Seong, K.-H. Chung, H. Park, Y. S. Kim, Ann. Phys. 2016, 528, 551.

[91] J. Ribeiro-Soares, C. Janisch, Z. Liu, A. L. Elías, M. S. Dresselhaus, M. Terrones, L. G. Cançado, A. Jorio, 2D Mater. 2015, 2, 045015. 
[92] X. Zhou, J. Cheng, Y. Zhou, T. Cao, H. Hong, Z. Liao, S. Wu, H. Peng, K. Liu, D. Yu, JACS 2015, 137, 7994.

[93]D. Li, W. Xiong, L. Jiang, Z. Xiao, H. Rabiee Golgir, M. Wang, X. Huang, Y. Zhou, Z. Lin, J. Song, S. Ducharme, L. Jiang, J.-F. Silvain, Y. Lu, ACS Nano 2016, 10, 3766.

[94] R. Wang, H.-C. Chien, J. Kumar, N. Kumar, H.-Y. Chiu, H. Zhao, ACS Appl. Mater. Interfaces 2014, 6, 314.

[95] A. Säynätjoki, L. Karvonen, H. Rostami, A. Autere, S. Mehravar, A. Lombardo, R.

Norwood, T. Hasan, N. Peyghambarian, H. Lipsanen, K. Kieu, A. Ferrari, M. Polini, Z. Sun, Nat. Commun. 2017, 8, 893.

[96]R. I. Woodward, R. T. Murray, C. F. Phelan, R. E. P. d. Oliveira, T. H. Runcorn, E. J. R. Kelleher, S. Li, E. C. d. Oliveira, G. J. M. Fechine, G. Eda, C. J. S. d. Matos, 2D Mater. 2017, 4, 011006.

[97]L. Karvonen, A. Säynätjoki, M. J. Huttunen, A. Autere, B. Amirsolaimani, R. A.

Norwood, N. Peyghambarian, H. Lipsanen, G. Eda, K. Kieu, Z. Sun, Nat. Commun. 2017, 8, 15714.

[98]Q. Cui, R. A. Muniz, J. E. Sipe, H. Zhao, Phys. Rev. B 2017, 95, 165406.

[99] L. Karvonen, A. Säynätjoki, S. Mehravar, R. D. Rodriguez, S. Hartmann, D. R. T. Zahn, S. Honkanen, R. A. Norwood, N. Peyghambarian, K. Kieu, H. Lipsanen, J. Riikonen, Sci. Rep. 2015, 5, 10334.

[100] N. Dong, Y. Li, Y. Feng, S. Zhang, X. Zhang, C. Chang, J. Fan, L. Zhang, J. Wang, Sci. Rep. 2015, 5, 14646.

[101] H. Liu, Y. Li, Y. S. You, S. Ghimire, T. F. Heinz, D. A. Reis, Nat. Phys. 2016, 13, 262.

[102] T. Jakubczyk, V. Delmonte, M. Koperski, K. Nogajewski, C. Faugeras, W.

Langbein, M. Potemski, J. Kasprzak, Nano Lett. 2016, 16, 5333.

[103] M. Zhao, Z. Ye, R. Suzuki, Y. Ye, H. Zhu, J. Xiao, Y. Wang, Y. Iwasa, X. Zhang, Light Sci. Appl. 2016, 5, e16131.

[104] J. Shi, P. Yu, F. Liu, P. He, R. Wang, L. Qin, J. Zhou, X. Li, J. Zhou, X. Sui, S. Zhang, Y. Zhang, Q. Zhang, T. C. Sum, X. Qiu, Z. Liu, X. Liu, Adv. Mater. 2017, 29, 1701486.

[105] G. Sallen, L. Bouet, X. Marie, G. Wang, C. R. Zhu, W. P. Han, Y. Lu, P. H. Tan, T. Amand, B. L. Liu, B. Urbaszek, Phys. Rev. B 2012, 86, 081301; T. Cao, G. Wang, W. Han, H. Ye, C. Zhu, J. Shi, Q. Niu, P. Tan, E. Wang, B. Liu, J. Feng, Nat. Commun. 2012, 3, 887;

K. F. Mak, K. He, J. Shan, T. F. Heinz, Nat. Nanotechnol. 2012, 7, 494; H. L. Zeng, J. F. Dai, W. Yao, D. Xiao, X. D. Cui, Nat. Nanotechnol. 2012, 7, 490; X. Xu, W. Yao, D. Xiao, T. F. Heinz, Nat. Phys. 2014, 10, 343.

[106] L. Li, Y. Yu, G. J. Ye, Q. Ge, X. Ou, H. Wu, D. Feng, X. H. Chen, Y. Zhang, Nat. Nanotechnol. 2014, 9, 372; H. Liu, A. T. Neal, Z. Zhu, Z. Luo, X. F. Xu, D. Tomanek, P. D. Ye, ACS Nano 2014, 8, 4033; S. P. Koenig, R. A. Doganov, H. Schmidt, A. H. Castro Neto, B. Özyilmaz, Appl. Phys. Lett. 2014, 104, 103106.

[107] V. Tran, R. Soklaski, Y. Liang, L. Yang, Phys. Rev. B 2014, 89, 235319; T. Low, A. S. Rodin, A. Carvalho, Y. Jiang, H. Wang, F. Xia, A. H. Castro Neto, Phys. Rev. B 2014, 90, 075434; J. Qiao, X. Kong, Z.-X. Hu, F. Yang, W. Ji, Nat. Commun. 2014, 5, 4475.

[108] L. Li, J. Kim, C. Jin, G. J. Ye, D. Y. Qiu, F. H. da Jornada, Z. Shi, L. Chen, Z. Zhang, F. Yang, K. Watanabe, T. Taniguchi, W. Ren, S. G. Louie, X. H. Chen, Y. Zhang, F. Wang, Nat. Nanotechnol. 2017, 12, 21.

[109] F. Xia, H. Wang, Y. Jia, Nat. Commun. 2014, 5, 4458. 
[110] H. Yang, H. Jussila, A. Autere, H.-P. Komsa, G. Ye, X. Chen, T. Hasan, Z. Sun, ACS Photon. 2017, DOI:10.1021/acsphotonics.7b00507.

[111] D. Li, H. Jussila, L. Karvonen, G. Ye, H. Lipsanen, X. Chen, Z. Sun, Sci. Rep. 2015, 5,15899 .

[112] Q. Wei, X. H. Peng, Appl. Phys. Lett. 2014, 104, 251915; Z. Luo, J. Maassen, Y. X. Deng, Y. C. Du, R. P. Garrelts, M. S. Lundstrom, P. D. Ye, X. F. Xu, Nat. Commun. 2015, 6, 8572 .

[113] X. Zheng, R. Chen, G. Shi, J. Zhang, Z. Xu, T. Jiang, Opt. Lett. 2015, 40, 3480.

[114] A. Autere, C. R. Ryder, A. Saynatjoki, L. Karvonen, B. Amirsolaimani, R. A.

Norwood, N. Peyghambarian, K. Kieu, H. Lipsanen, M. C. Hersam, Z. Sun, J. Phys. Chem. Lett. 2017, 8, 1343.

[115] N. Youngblood, R. Peng, A. Nemilentsau, T. Low, M. Li, ACS Photon. 2016, $4,8$.

[116] S. Uddin, P. C. Debnath, K. Park, Y.-W. Song, Sci. Rep. 2017, 7, 43371.

[117] J. Zheng, Z. Yang, C. Si, Z. Liang, X. Chen, R. Cao, Z. Guo, K. Wang, Y. Zhang, J.

Ji, M. Zhang, D. Fan, H. Zhang, ACS Photon. 2017, 4, 1466.

[118] D. Li, A. E. Del Rio Castillo, H. Jussila, G. Ye, Z. Ren, J. Bai, X. Chen, H.

Lipsanen, Z. Sun, F. Bonaccorso, Appl. Mater. Today 2016, 4, 17.

[119] G. H. Hu, T. Albrow-Owen, X. X. Jin, A. Ali, Y. W. Hu, R. C. T. Howe, K.

Shehzad, Z. Y. Yang, X. K. Zhu, R. I. Woodward, T. C. Wu, H. Jussila, J. B. Wu, P. Peng, P.

H. Tan, Z. P. Sun, E. J. R. Kelleher, M. Zhang, Y. Xu, T. Hasan, Nat. Commun. 2017, 8, 278.

[120] J. D. Wood, S. A. Wells, D. Jariwala, K.-S. Chen, E. Cho, V. K. Sangwan, X. Liu, L. J. Lauhon, T. J. Marks, M. C. Hersam, Nano Lett. 2014, 14, 6964.

[121] J. O. Island, G. A. Steele, H. S. van der Zant, A. Castellanos-Gomez, 2D Mater.

2015, 2, 011002; Y. Zhao, H. Wang, H. Huang, Q. Xiao, Y. Xu, Z. Guo, H. Xie, J. Shao, Z.

Sun, W. Han, X. F. Yu, P. Li, P. K. Chu, Angew. Chem. Int. Ed. 2016, 55, 5003; Y. Y.

Illarionov, M. Waltl, G. Rzepa, J. S. Kim, S. Kim, A. Dodabalapur, D. Akinwande, T.

Grasser, ACS Nano 2016, 10, 9543.

[122] C. R. Dean, A. F. Young, I. Meric, C. Lee, L. Wang, S. Sorgenfrei, K. Watanabe, T. Taniguchi, P. Kim, K. L. Shepard, J. Hone, Nat. Nanotechnol. 2010, 5, 722.

[123] T. T. Tran, K. Bray, M. J. Ford, M. Toth, I. Aharonovich, Nat. Nanotechnol. 2015, 11,37 .

[124] R. Bourrellier, S. Meuret, A. Tararan, O. Stephan, M. Kociak, L. H. Tizei, A. Zobelli, Nano Lett. 2016, 16, 4317.

[125] C. Kurtsiefer, S. Mayer, P. Zarda, H. Weinfurter, Phys. Rev. Lett. 2000, 85, 290.

[126] J. Susoma, L. Karvonen, A. Säynätjoki, S. Mehravar, R. A. Norwood, N.

Peyghambarian, K. Kieu, H. Lipsanen, J. Riikonen, Appl. Phys. Lett. 2016, 108, 073103.

[127] H. Wang, X. Qian, Nano Lett. 2017, 17, 5027.

[128] F. Giorgianni, E. Chiadroni, A. Rovere, M. Cestelli-Guidi, A. Perucchi, M.

Bellaveglia, M. Castellano, D. Di Giovenale, G. Di Pirro, M. Ferrario, R. Pompili, C.

Vaccarezza, F. Villa, A. Cianchi, A. Mostacci, M. Petrarca, M. Brahlek, N. Koirala, S. Oh, S. Lupi, Nat. Commun. 2016, 7, 11421.

[129] L. Wu, S. Patankar, T. Morimoto, N. L. Nair, E. Thewalt, A. Little, J. G. Analytis, J. E. Moore, J. Orenstein, Nat. Phys. 2016, 13, 350.

[130] J. Mertens, A. L. Eiden, D. O. Sigle, F. M. Huang, A. Lombardo, Z. P. Sun, R. S.

Sundaram, A. Colli, C. Tserkezis, J. Aizpurua, S. Milana, A. C. Ferrari, J. J. Baumberg, Nano Lett. 2013, 13, 5033.

[131] D. Jariwala, T. J. Marks, M. C. Hersam, Nat. Mater. 2017, 16, 170. 
[132] T. J. Echtermeyer, P. Nene, M. Trushin, R. V. Gorbachev, A. Eiden, S. Milana, Z. Sun, J. Schliemann, E. Lidorikis, K. S. Novoselov, A. C. Ferrari, Nano Lett. 2014, 14, 3733. [133] A. K. Geim, I. V. Grigorieva, Nature 2013, 499, 419; K. S. Novoselov, A. Mishchenko, A. Carvalho, A. H. Castro Neto, Science 2016, 353, 6298.

[134] V. O. Özçelik, J. G. Azadani, C. Yang, S. J. Koester, T. Low, Phys. Rev. B 2016, 94, 035125 .

[135] F. Ceballos, M. Z. Bellus, H.-Y. Chiu, H. Zhao, ACS Nano 2014, 8, 12717.

[136] K. L. Seyler, J. R. Schaibley, P. Gong, P. Rivera, A. M. Jones, S. Wu, J. Yan, D. G. Mandrus, W. Yao, X. Xu, Nat. Nanotechnol. 2015, 10, 407.

[137] W.-T. Hsu, Z.-A. Zhao, L.-J. Li, C.-H. Chen, M.-H. Chiu, P.-S. Chang, Y.-C. Chou, W.-H. Chang, ACS Nano 2014, 8, 2951.

[138] K. Liu, L. Zhang, T. Cao, C. Jin, D. Qiu, Q. Zhou, A. Zettl, P. Yang, S. G. Louie, F. Wang, Nat. Commun. 2014, 5, 4966; A. M. van der Zande, J. Kunstmann, A. Chernikov, D.

A. Chenet, Y. You, X. Zhang, P. Y. Huang, T. C. Berkelbach, L. Wang, F. Zhang, M. S. Hybertsen, D. A. Muller, D. R. Reichman, T. F. Heinz, J. C. Hone, Nano Lett. 2014, 14, 3869; L. Zhang, K. Liu, A. B. Wong, J. Kim, X. Hong, C. Liu, T. Cao, S. G. Louie, F. Wang, P. Yang, Nano Lett. 2014, 14, 6418.

[139] T. Jiang, H. Liu, D. Huang, S. Zhang, Y. Li, X. Gong, Yuen-Ron Shen, W.-T. Liu, S. Wu, Nat. Nanotechnol. 2014, 9, 825.

[140] H. Chen, J. Yin, J. Yang, X. Zhang, M. liu, Z. Jiang, J. Wang, Z. Sun, T. Guo, W. Liu, P. Yan, Opt. Lett. 2017, 42, 4279.

[141] M. J. L. F. Rodrigues, C. J. S. de Matos, Y. W. Ho, H. Peixoto, R. E. P. de Oliveira, H.-Y. Wu, A. H. C. Neto, J. Viana-Gomes, Adv. Mater. 2016, 28, 10693.

[142] C. T. Le, D. J. Clark, F. Ullah, J. I. Jang, V. Senthilkumar, Y. Sim, M.-J. Seong, K.H. Chung, J. W. Kim, S. Park, S. H. Rhim, G. Kim, Y. S. Kim, ACS Photon. 2017, 4, 38.

[143] W. Jie, X. Chen, D. Li, L. Xie, Y. Y. Hui, S. P. Lau, X. Cui, J. Hao, Angew. Chem. Int. Ed. 2015, 54, 1185.

[144] C.-J. Kim, L. Brown, M. W. Graham, R. Hovden, R. W. Havener, P. L. McEuen, D. A. Muller, J. Park, Nano Lett. 2013, 13, 5660.

[145] S. H. Rhim, Y. S. Kim, A. J. Freeman, Appl. Phys. Lett. 2015, 107, 241908.

[146] S. Deckoff-Jones, J. Zhang, C. E. Petoukhoff, M. K. L. Man, S. Lei, R. Vajtai, P. M. Ajayan, D. Talbayev, J. Madéo, K. M. Dani, Sci. Rep. 2016, 6, 22620.

[147] X. Wang, K. Du, W. Liu, P. Hu, X. Lu, W. Xu, C. Kloc, Q. Xiong, Appl. Phys. Lett. 2016, 109, 123103.

[148] B. Xu, A. Martinez, S. Yamashita, IEEE Photonics Technol. Lett. 2012, 24, 1792.

[149] S. Yamashita, J. Lightwave Technol. 2012, 30, 427.

[150] J. Klein, J. Wierzbowski, A. Steinhoff, M. Florian, M. Rösner, F. Heimbach, K.

Müller, F. Jahnke, T. O. Wehling, J. J. Finley, M. Kaniber, Nano Lett. 2017, 17, 392.

[151] H. Yu, D. Talukdar, W. Xu, J. B. Khurgin, Q. Xiong, Nano Lett. 2015, 15, 5653.

[152] X. Fan, Y. Jiang, X. Zhuang, H. Liu, T. Xu, W. Zheng, P. Fan, H. Li, X. Wu, X.

Zhu, Q. Zhang, H. Zhou, W. Hu, X. Wang, L. Sun, X. Duan, A. Pan, ACS Nano 2017, 10.1021/acsnano.7b01457.

[153] D. J. Clark, C. T. Le, V. Senthilkumar, F. Ullah, H.-Y. Cho, Y. Sim, M.-J. Seong,

K.-H. Chung, Y. S. Kim, J. I. Jang, Appl. Phys. Lett. 2015, 107, 131113.

[154] B. Radisavljevic, A. Radenovic, J. Brivio, i. V. Giacometti, A. Kis, Nat.

Nanotechnol. 2011, 6, 147.

[155] R. L. Sutherland, Handbook of Nonlinear Optics, New York 2003. 
[156] Y. Q. An, J. E. Rowe, D. B. Dougherty, J. U. Lee, A. C. Diebold, Phys. Rev. B 2014, 89, 115310.

[157] H. Chen, V. Corboliou, A. S. Solntsev, D.-Y. Choi, M. A. Vincenti, D. d. Ceglia, C. D. Angelis, Y. Lu, D. N.Neshev, Light Sci. Appl. 2017, 6, e17060.

[158] K. F. Taylor, L. S. Kyle, Z. Jiajiu, L. Chang-Hua, X. Xiaodong, M. Arka, 2D Mater. 2017, 4, 015031; J. K. Day, M.-H. Chung, Y.-H. Lee, V. M. Menon, Opt. Mater. Express 2016, 6, 2360.

[159] G. M. Akselrod, T. Ming, C. Argyropoulos, T. B. Hoang, Y. Lin, X. Ling, D. R. Smith, J. Kong, M. H. Mikkelsen, Nano Lett. 2015, 15, 3578.

[160] X. Gan, C. Zhao, S. Hu, T. Wang, Y. Song, J. Li, Q. Zhao, W. Jie, J. Zhao, arXiv 2017, 1706.07923.

[161] X. Miao, N. Xuan, Q. Liu, W. Wu, H. Liu, Z. Sun, M. Ji, ACS Appl. Mater. Interfaces 2017, DOI: 10.1021/acsami.7b09807.

[162] J. Zeng, M. Yuan, W. Yuan, Q. Dai, H. Fan, S. Lan, S. Tie, Nanoscale 2015, 7, 13547.

[163] J. L. Cheng, N. Vermeulen, J. E. Sipe, New J. Phys. 2014, 16, 053014.

[164] H. Shi, C. Wang, Z. Sun, Y. Zhou, K. Jin, S. A. T. Redfern, G. Yang, Opt. Express 2014, 22, 19375.

[165] H. Nan, Z. Wang, W. Wang, Z. Liang, Y. Lu, Q. Chen, D. He, P. Tan, F. Miao, X. Wang, J. Wang, Z. Ni, ACS Nano 2014, 8, 5738.

[166] J. Liang, J. Zhang, Z. Li, H. Hong, J. Wang, Z. Zhang, X. Zhou, R. Qiao, J. Xu, P. Gao, Z. Liu, Z. Liu, Z. Sun, S. Meng, K. Liu, D. Yu, Nano Lett. 2017,

DOI:10.1021/acs.nanolett.7b03476.

[167] F. Krausz, M. Ivanov, Rev. Mod. Phys. 2009, 81, 163.

[168] C. Hernandez-Garcia, J. A. Perez-Hernandez, T. Popmintchev, M. M. Murnane, H. C. Kapteyn, A. Jaron-Becker, A. Becker, L. Plaja, Phys. Rev. Lett. 2013, 111, 033002.

[169] T. Popmintchev, M.-C. Chen, P. Arpin, M. M. Murnane, H. C. Kapteyn, Nat. Photonics 2010, 4, 822; A. McPherson, G. Gibson, H. Jara, U. Johann, T. S. Luk, A. I.

McIntyre, K. Boyer, C. K. Rhodes, J. Opt. Soc. Am. B 1987, 4, 595.

[170] S. Ghimire, A. D. DiChiara, E. Sistrunk, P. Agostini, L. F. DiMauro, D. A. Reis, Nat. Phys. 2010, 7, 138.

[171] M. Hohenleutner, F. Langer, O. Schubert, M. Knorr, U. Huttner, S. W. Koch, M.

Kira, R. Huber, Nature 2015, 523, 572.

[172] F. Langer, M. Hohenleutner, C. P. Schmid, C. Poellmann, P. Nagler, T. Korn, C.

Schüller, M. S. Sherwin, U. Huttner, J. T. Steiner, S. W. Koch, M. Kira, R. Huber, Nature 2016, 533, 225.

[173] P. G. Hawkins, J. Steiner, U. Huttner, F. Langer, C. Schmid, S. Schlauderer, S. W. Koch, R. Huber, M. Kira, "Harmonic Sideband Generation in Monolayer Transition Metal Dichalcogenides", presented at Conference on Lasers and Electro-Optics, San Jose, California, 2017. FM3D.7.

[174] I. Al-Naib, J. E. Sipe, M. M. Dignam, Phys. Rev. B 2014, 90, 245423.

[175] H. Zhou, T. Gu, J. F. McMillan, N. Petrone, A. van der Zande, J. C. Hone, M. Yu, G. Lo, D.-L. Kwong, G. Feng, S. Zhou, C. W. Wong, Appl. Phys. Lett. 2014, 105, 091111.

[176] J. Wang, X. Hu, J. Nanotechnol. 2016, 2016, 7031913; R. Ciesielski, A. Comin, M. Handloser, K. Donkers, G. Piredda, A. Lombardo, A. C. Ferrari, A. Hartschuh, Nano Lett. 2015, 15, 4968.

[177] M. Ji, H. Cai, L. Deng, Y. Huang, Q. Huang, J. Xia, Z. Li, J. Yu, Y. Wang, Opt. Express 2015, 23, 18679. 
[178] Y. Wu, B. Yao, Y. Cheng, Y. Rao, Y. Gong, X. Zhou, B. Wu, K. S. Chiang, IEEE Photonics Technol. Lett. 2013, 26, 249.

[179] L. Guo, W. Hou, H. B. Zhang, Z. P. Sun, D. F. Cui, Z. Y. Xu, Y. G. Wang, X. Y. Ma, Opt. Express 2005, 13, 4085.

[180] R. I. Woodward, E. J. R. Kelleher, R. C. T. Howe, G. Hu, F. Torrisi, T. Hasan, S. V. Popov, J. R. Taylor, Opt. Express 2014, 22, 31113; Y. Huang, Z. Luo, Y. Li, M. Zhong, B.

Xu, K. Che, H. Xu, Z. Cai, J. Peng, J. Weng, Opt. Express 2014, 22, 25258; H. Zhang, S. B. Lu, J. Zheng, J. Du, S. C. Wen, D. Y. Tang, K. P. Loh, Opt. Express 2014, 22, 7249.

[181] Z. Luo, D. Wu, B. Xu, H. Xu, Z. Cai, J. Peng, J. Weng, S. Xu, C. Zhu, F. Wang, Z. Sun, H. Zhang, Nanoscale 2016, 8, 1066.

[182] S. F. Zhang, N. N. Dong, N. McEvoy, M. O'Brien, S. Winters, N. C. Berner, C. Yim, Y. X. Li, X. Y. Zhang, Z. H. Chen, L. Zhang, G. S. Duesberg, J. Wang, ACS Nano 2015, 9, 7142; D. Mao, Y. Wang, C. Ma, L. Han, B. Jiang, X. Gan, S. Hua, W. Zhang, T. Mei, J. Zhao, Sci. Rep. 2015, 5, 7965; P. Yan, H. Chen, J. Yin, Z. Xu, J. Li, Z. Jiang, W. Zhang, J. Wang, I. L. Li, Z. Sun, S. Ruan, Nanoscale 2017, 9, 1871.

[183] J. Yin, J. Li, H. Chen, J. Wang, P. Yan, M. Liu, W. Liu, W. Lu, Z. Xu, W. Zhang, J. Wang, Z. Sun, S. Ruan, Opt. Express 2017, 25, 30020.

[184] K. Wang, Y. Feng, C. Chang, J. Zhan, C. Wang, Q. Zhao, J. N. Coleman, L. Zhang, W. J. Blau, J. Wang, Nanoscale 2014, 6, 10530.

[185] Y. Cui, F. Lu, X. Liu, Sci. Rep. 2017, 7, 40080.

[186] Y. Wang, G. Huang, H. Mu, S. Lin, J. Chen, S. Xiao, Q. Bao, J. He, Appl. Phys.

Lett. 2015, 107, 091905; Y. Chen, G. Jiang, S. Chen, Z. Guo, X. Yu, C. Zhao, H. Zhang, Q.

Bao, S. Wen, D. Tang, D. Fan, Opt. Express 2015, 23, 12823; J. Sotor, G. Sobon, W.

Macherzynski, P. Paletko, K. M. Abramski, Appl. Phys. Lett. 2015, 107, 051108.

[187] W. D. Tan, C. Y. Su, R. J. Knize, G. Q. Xie, L. J. Li, D. Y. Tang, Appl. Phys. Lett. 2010, 96, 031106; A. A. Lagatsky, Z. Sun, T. S. Kulmala, R. S. Sundaram, S. Milana, F. Torrisi, O. L. Antipov, Y. Lee, J. H. Ahn, C. T. A. Brown, W. Sibbett, A. C. Ferrari, Appl. Phys. Lett. 2013, 102, 013113; L. Li, X. Zheng, C. Jin, M. Qi, X. Chen, Z. Ren, J. Bai, Z. Sun, Appl. Phys. Lett. 2014, 105, 221103; L. Li, X. Zheng, X. Chen, M. Qi, Z. Ren, J. Bai, Z. Sun, Opt. Commun. 2014, 315, 204; L. Li, Z. Ren, X. Chen, M. Qi, X. Zheng, J. Bai, Z. Sun, Appl. Phys. Express 2013, 6, 082701.

[188] R. Mary, G. Brown, S. J. Beecher, F. Torrisi, S. Milana, D. Popa, T. Hasan, Z. P. Sun, E. Lidorikis, S. Ohara, A. C. Ferrari, A. K. Kar, Opt. Express 2013, 21, 7943.

[189] D. Popa, Z. Sun, T. Hasan, F. Torrisi, F. Wang, A. C. Ferrari, Appl. Phys. Lett. 2011, 98, 073106.

[190] D. Popa, Z. Sun, F. Torrisi, T. Hasan, F. Wang, A. C. Ferrari, Appl. Phys. Lett. 2010, 97, 203106; X. Li, X. Yu, Z. Sun, Z. Yan, B. Sun, Y. Cheng, X. Yu, Y. Zhang, Q. J. Wang, Sci. Rep. 2015, 5, 16624; T. Hasan, F. Torrisi, Z. Sun, D. Popa, V. Nicolosi, G. Privitera, F. Bonaccorso, A. C. Ferrari, Phys. Stat. Sol. (b) 2010, 247, 2953; M. Zhang, E. J. R. Kelleher, F. Torrisi, Z. Sun, T. Hasan, D. Popa, F. Wang, A. C. Ferrari, S. V. Popov, J. R. Taylor, Opt. Express 2012, 20, 25077.

[191] C. A. Zaugg, Z. Sun, V. J. Wittwer, D. Popa, S. Milana, T. S. Kulmala, R. S. Sundaram, M. Mangold, O. D. Sieber, M. Golling, Y. Lee, J. H. Ahn, A. C. Ferrari, U. Keller, Opt. Express 2013, 21, 31548.

[192] F. Canbaz, N. Kakenov, C. Kocabas, U. Demırbas, A. Sennaroglu, Opt. Express 2017, 25, 2834.

[193] A. Martinez, S. Yamashita, Appl. Phys. Lett. 2012, 101, 041118.

[194] S. Husaini, R. G. Bedford, Appl. Phys. Lett. 2014, 104, 161107. 
[195] J. H. Chen, B. C. Zheng, G. H. Shao, S. J. Ge, F. Xu, Y. Q. Lu, Light Sci. Appl. 2015, 4, e360; Z. Shi, L. Gan, T. H. Xiao, H. L. Guo, Z. Y. Li, ACS Photon. 2015, 2, 1513. [196] M. G. Kuzyk, Phys. Rev. Lett. 2000, 85, 1218; M. G. Kuzyk, K. D. Singer, G. I. Stegeman, Adv. Opt. Photonics 2013, 5, 4; R. Lytel, J. Opt. Soc. Am. B 2016, 33, E66.

[197] S. A. Mikhailov, Europhys. Lett. 2007, 79, 27002.

[198] M. M. Glazov, Jetp Lett. 2011, 93, 366; A. Y. Bykov, T. V. Murzina, M. G. Rybin, E. D. Obraztsova, Phys. Rev. B 2012, 85, 121413; L. E. Golub, S. A. Tarasenko, Phys. Rev. B 2014, 90, 201402.

[199] J. B. Khurgin, Appl. Phys. Lett. 2014, 104, 161116.

[200] X. Yin, Z. Ye, D. A. Chenet, Y. Ye, K. O’Brien, J. C. Hone, X. Zhang, Science 2014, 344, 488.

[201] M. Grüning, C. Attaccalite, Phys. Rev. B 2014, 89, 081102.

[202] D. J. Clark, V. Senthilkumar, C. T. Le, D. L. Weerawarne, B. Shim, J. I. Jang, J. H. Shim, J. Cho, Y. Sim, M. J. Seong, S. H. Rhim, A. J. Freeman, K. H. Chung, Y. S. Kim, Phys. Rev. B 2014, 90, 121409.

[203] A. Autere, A. Säynätjoki, L. Karvonen, B. Amirsolaimani, H. Jussila, H. Yang, R. Norwood, N. Peyghambarian, H. Lipsanen, K. Q. Kieu, Z. Sun, "Direct comparison of second and third harmonic generation in mono- and few-layer MX2 $(\mathrm{M}=\mathrm{Mo}, \mathrm{W} ; \mathrm{X}=\mathrm{S}, \mathrm{Se})$ by multiphoton microscope", presented at Conference on Lasers and Electro-Optics, San Jose, USA, 2016. FTu1A.2; A. Autere, H. Jussila, A. Marini, J. R. M. Saavedra, A. Saynatjoki, L. Karvonen, H. Yang, B. Amirsolaimani, R. A. Norwood, N. Peyghambarian, H. Lipsanen, K. Kieu, F. J. G. d. Abajo, Z. Sun, Under preparation 2018.

[204] Y. Li, A. Chernikov, X. Zhang, A. Rigosi, H. M. Hill, A. M. van der Zande, D. A. Chenet, E.-M. Shih, J. Hone, T. F. Heinz, Phys. Rev. B 2014, 90, 205422; C.-Y. Wang, G.-Y. Guo, J. Phys. Chem. C 2015, 119, 13268.

[205] J. Cheng, T. Jiang, Q. Ji, Y. Zhang, Z. Li, Y. Shan, Y. Zhang, X. Gong, W. Liu, S. Wu, Adv. Mater. 2015, 27, 4069.

[206] A. Srivastava, M. Sidler, A. V. Allain, D. S. Lembke, A. Kis, A. Imamoglu, Nat. Nanotechnol. 2015, 10, 491; Y. M. He, G. Clark, J. R. Schaibley, Y. He, M. C. Chen, Y. J. Wei, X. Ding, Q. Zhang, W. Yao, X. Xu, C. Y. Lu, J. W. Pan, Nat. Nanotechnol. 2015, 10, 497; M. Koperski, K. Nogajewski, A. Arora, V. Cherkez, P. Mallet, J. Y. Veuillen, J. Marcus, P. Kossacki, M. Potemski, Nat. Nanotechnol. 2015, 10, 503; C. Chakraborty, L.

Kinnischtzke, K. M. Goodfellow, R. Beams, A. N. Vamivakas, Nat. Nanotechnol. 2015, 10, 507.

[207] Q. Liu, B. Guo, Z. Rao, B. Zhang, J. R. Gong, Nano Lett. 2013, 13, 2436.

[208] J. L. Li, H. C. Bao, X. L. Hou, L. Sun, X. G. Wang, M. Gu, Angew. Chem. Int. Ed. 2012, 51, 1830 .

[209] A. Ananthanarayanan, Y. Wang, P. Routh, M. A. Sk, A. Than, M. Lin, J. Zhang, J. Chen, H. Sun, P. Chen, Nanoscale 2015, 7, 8159.

[210] A. Pramanik, S. R. Chavva, Z. Fan, S. S. Sinha, B. P. Nellore, P. C. Ray, J. Phys. Chem. Lett. 2014, 5, 2150.

[211] H. Dong, S. Tang, Y. Hao, H. Yu, W. Dai, G. Zhao, Y. Cao, H. Lu, X. Zhang, H. Ju, ACS Appl. Mater. Interfaces 2016, 8, 3107.

[212] W. Dai, H. Dong, B. Fugetsu, Y. Cao, H. Lu, X. Ma, X. Zhang, Small 2015, 11, 4158.

[213] J. Lin, X. Chen, P. Huang, Adv. Drug Delivery Rev. 2016, 105, 242; X. Zhang, H. Cheng, H. Zhang, Adv. Mater. 2017, 29; J. M. Yoo, J. H. Kang, B. H. Hong, Chem. Soc. 
Rev. 2015, 44, 4835; A. Pramanik, Z. Fan, S. R. Chavva, S. S. Sinha, P. C. Ray, Sci. Rep. 2014, 4, 6090 .

[214] J. D. Cox, F. J. García de Abajo, ACS Photon. 2015, 2, 306.

[215] J. D. Cox, I. Silveiro, F. J. García de Abajo, ACS Nano 2016, 10, 1995.

[216] J. D. Cox, A. Marini, F. J. García de Abajo, Nat. Commun. 2017, 8, 14380.

[217] J. D. Cox, R. Yu, F. J. G. de Abajo, Phys. Rev. B 2017, 96, 045442.

[218] R. Yu, J. D. Cox, F. J. de Abajo, Phys. Rev. Lett. 2016, 117, 123904.

[219] A. Autere, L. Karvonen, A. Säynätjoki, M. Roussey, E. Färm, M. Kemell, X. Tu, T. Y. Liow, G. Q. Lo, M. Ritala, M. Leskelä, S. Honkanen, H. Lipsanen, Z. Sun, Opt. Express 2015, 23, 26940.

[220] X.-C. Zhang, J. Xu, Introduction to THz wave photonics, Vol. 29, Springer, 2010.

[221] R. R. Hartmann, J. Kono, M. E. Portnoi, Nanotechnol. 2014, 25, 322001.

[222] G. Ramakrishnan, R. Chakkittakandy, P. C. M. Planken, Opt. Express 2009, 17, 16092.

[223] C. W. Luo, H.-J. Chen, C. M. Tu, C. C. Lee, S. A. Ku, W. Y. Tzeng, T. T. Yeh, M. C. Chiang, H. J. Wang, W. C. Chu, J.-Y. Lin, K. H. Wu, J. Y. Juang, T. Kobayashi, C.-M. Cheng, C.-H. Chen, K.-D. Tsuei, H. Berger, R. Sankar, F. C. Chou, H. D. Yang, Adv. Opt. Mater. 2013, 1, 804.

[224] Y. Huang, L. Zhu, Q. Zhao, Y. Guo, Z. Ren, J. Bai, X. Xu, ACS Appl. Mater. Interfaces 2017, 9, 4956.

[225] L. Zhang, Y. Huang, Q. Zhao, L. Zhu, Z. Yao, Y. Zhou, W. Du, X. Xu, Phys. Rev. B 2017, 96, 155202.

[226] J. Maysonnave, S. Huppert, F. Wang, S. Maero, C. Berger, W. de Heer, T. B. Norris, L. A. De Vaulchier, S. Dhillon, J. Tignon, R. Ferreira, J. Mangeney, Nano Lett. 2014, 14, 5797.

[227] L. Prechtel, L. Song, D. Schuh, P. Ajayan, W. Wegscheider, A. W. Holleitner, Nat. Commun. 2012, 3, 646; P. Bowlan, E. Martinez-Moreno, K. Reimann, T. Elsaesser, M. Woerner, Phys. Rev. B 2014, 89, 041408; Z. Mics, K. J. Tielrooij, K. Parvez, S. A. Jensen, I. Ivanov, X. Feng, K. Mullen, M. Bonn, D. Turchinovich, Nat. Commun. 2015, 6, 7655.

[228] P. A. Obraztsov, N. Kanda, K. Konishi, M. Kuwata-Gonokami, S. V. Garnov, A. N. Obraztsov, Y. P. Svirko, Phys. Rev. B 2014, 90, 241416.

[229] S. M. Raeis-Zadeh, B. Semnani, S. Safavi-Naeini, Phys. Rev. B 2016, 94, 121411.

[230] T. Ye, S. Meng, J. Zhang, Y. E, Y. Yang, W. Liu, Y. Yin, L. Wang, Sci. Rep. 2016, 6, 22798.

[231] T. J. Constant, S. M. Hornett, D. E. Chang, E. Hendry, Nat. Phys. 2015, 12, 124.

[232] J. Perruisseau-Carrier, "Graphene for antenna applications: Opportunities and challenges from microwaves to THz", presented at Antennas and Propagation Conference (LAPC), 2012 Loughborough, 2012. 13255996.

[233] M. O. Scully, M. S. Zubairy, Quantum Optics, Cambridge 1997.

[234] C. K. Hong, L. Mandel, Phys. Rev. Lett. 1986, 56, 58.

[235] B. Darquié, M. P. Jones, J. Dingjan, J. Beugnon, S. Bergamini, Y. Sortais, G.

Messin, A. Browaeys, P. Grangier, Science 2005, 309, 454.

[236] I. Aharonovich, S. Castelletto, D. A. Simpson, C. H. Su, A. D. Greentree, S. Prawer, Rep. Prog. Phys. 2011, 74, 076501.

[237] C. Santori, M. Pelton, G. Solomon, Y. Dale, Y. Yamamoto, Phys. Rev. Lett. 2001, $86,1502$.

[238] P. G. Kwiat, K. Mattle, H. Weinfurter, A. Zeilinger, A. V. Sergienko, Y. Shih, Phys. Rev. Lett. 1995, 75, 4337. 
[239] Q. Lin, F. Yaman, G. P. Agrawal, Phys. Rev. A 2007, 75, 023803; K. F. Lee, Y. Tian, H. Yang, K. Mustonen, A. Martinez, Q. Dai, E. I. Kauppinen, J. Malowicki, P. Kumar, Z. Sun, Adv. Mater. 2017, 29, 1605978.

[240] M. Gullans, D. E. Chang, F. H. L. Koppens, F. J. G. de Abajo, M. D. Lukin, Phys. Rev. Lett. 2013, 111, 247401.

[241] M. Hentschel, R. Kienberger, C. Spielmann, G. A. Reider, N. Milosevic, T. Brabec, P. Corkum, U. Heinzmann, M. Drescher, F. Krausz, Nature 2001, 414, 509; A. Baltuška, T. Udem, M. Uiberacker, M. Hentschel, E. Goulielmakis, C. Gohle, R. Holzwarth, V. Yakovlev, A. Scrinzi, T. W. Hänsch, Nature 2003, 421, 611; A. Fohlisch, P. Feulner, F. Hennies, A. Fink, D. Menzel, D. Sanchez-Portal, P. M. Echenique, W. Wurth, Nature 2005, 436, 373. [242] P. M. Paul, E. Toma, P. Breger, G. Mullot, F. Augé, P. Balcou, H. Muller, P. Agostini, Science 2001, 292, 1689.

[243] G. Herink, D. R. Solli, M. Gulde, C. Ropers, Nature 2012, 483, 190; M. Kruger, M. Schenk, P. Hommelhoff, Nature 2011, 475, 78; A. Feist, K. E. Echternkamp, J. Schauss, S. V. Yalunin, S. Schafer, C. Ropers, Nature 2015, 521, 200; C. Li, X. Zhou, F. Zhai, Z. Li, F. Yao, R. Qiao, K. Chen, M. T. Cole, D. Yu, Z. Sun, K. Liu, Q. Dai, Adv. Mater. 2017, 29, 1701580; C. Li, X. Zhou, F. Zhai, Z. Li, F. Yao, R. Qiao, K. Chen, D. Yu, Z. Sun, K. Liu, Q. Dai, Appl. Phys. Lett. 2017, 111, 133101.

[244] T. Higuchi, C. Heide, K. Ullmann, H. B. Weber, P. Hommelhoff, Nature 2017, 550, 224.

[245] J. E. Lommerts, M. D. Njoo, M. A. de Rie, A. Wolkerstorfer, M. W. Bekkenk, British Journal of Dermatology 2017, 177, e60.

[246] C. Wagner, N. Harned, Nat. Photonics 2010, 4, 24; G. L. Wang, A. C. Geng, Y. Bo, H. Q. Li, Z. P. Sun, Y. Bi, D. F. Cui, Z. Y. Xu, X. Yuan, X. Q. Wang, G. Q. Shen, D. Z. Shen, Opt. Commun. 2006, 259, 820.

[247] G. Cassabois, P. Valvin, B. Gil, Nat. Photonics 2016, 10, 262.

[248] A. C. Geng, Y. Bo, X. D. Yang, H. Q. Li, Z. P. Sun, Q. J. Peng, X. J. Wang, G. L. Wang, D. F. Cui, Z. Y. Xu, Opt. Commun. 2005, 255, 248.

[249] W. Strek, R. Tomala, M. Lukaszewicz, B. Cichy, Y. Gerasymchuk, P. Gluchowski, L. Marciniak, A. Bednarkiewicz, D. Hreniak, Sci. Rep. 2017, 7, 41281; W. Strek, B. Cichy, L. Radosinski, P. Gluchowski, L. Marciniak, M. Lukaszewicz, D. Hreniak, Light Sci. Appl. 2015, 4, e237.

[250] C. H. Lui, K. F. Mak, J. Shan, T. F. Heinz, Phys. Rev. Lett. 2010, 105, 127404; R. J. Stohr, R. Kolesov, J. Pflaum, J. Wrachtrup, Phys. Rev. B 2010, 82, 121408.

[251] G. Cerullo, S. De Silvestri, Rev. Sci. Instrum. 2003, 74, 1.

[252] H. Q. Li, H. B. Zhang, Z. Bao, J. Zhang, Z. P. Sun, Y. P. Kong, Y. Bi, X. C. Lin, A. Y. Yao, G. L. Wang, W. Hou, R. N. Li, D. F. Cui, Z. Y. Xu, Opt. Commun. 2004, 232, 411.

[253] A. Ciattoni, A. Marini, C. Rizza, C. Conti, arXiv 2017, 1707.08843.

[254] L. Razzari, D. Duchesne, M. Ferrera, R. Morandotti, S. Chu, B. E. Little, D. J. Moss, Nat. Photonics 2009, 4, 41.

[255] S. Wu, S. Buckley, J. R. Schaibley, L. Feng, J. Yan, D. G. Mandrus, F. Hatami, W. Yao, J. Vuckovic, A. Majumdar, X. Xu, Nature 2015, 520, 69; Y. Ye, Z. J. Wong, X. Lu, X. Ni, H. Zhu, X. Chen, Y. Wang, X. Zhang, Nat. Photonics 2015, 9, 733; O. Salehzadeh, M. Djavid, N. H. Tran, I. Shih, Z. Mi, Nano Lett. 2015, 15, 5302.

[256] A. N. Grigorenko, M. Polini, K. S. Novoselov, Nat. Photonics 2012, 6, 749.

[257] J. D. Caldwell, A. V. Kretinin, Y. Chen, V. Giannini, M. M. Fogler, Y. Francescato, C. T. Ellis, J. G. Tischler, C. R. Woods, A. J. Giles, M. Hong, K. Watanabe, T. Taniguchi, S. A. Maier, K. S. Novoselov, Nat. Commun. 2014, 5, 5221. 
[258] J. Xiao, M. Zhao, Y. Wang, X. Zhang, Nanophotonics 2017, 6, 1309.

[259] H. Hu, X. Yang, F. Zhai, D. Hu, R. Liu, K. Liu, Z. Sun, Q. Dai, Nat. Commun. 2016, 7, 12334 .

[260] D. Kundys, B. Van Duppen, O. P. Marshall, F. Rodriguez, I. Torre, M. Polini, A. N. Grigorenko, arXiv 2017, 1705.09739.

[261] X. X. Yang, F. Zhai, H. Hu, D. B. Hu, R. N. Liu, S. P. Zhang, M. T. Sun, Z. P. Sun, J. N. Chen, Q. Dai, Adv. Mater. 2016, 28, 2931; D. Hu, X. Yang, C. Li, R. Liu, Z. Yao, H.

Hu, S. N. G. Corder, J. Chen, Z. Sun, M. Liu, Q. Dai, Nat. Commun. 2017, 8, 1471.

[262] C. Monat, M. de Sterke, B. J. Eggleton, J. Opt. 2010, 12, 104003.

[263] C. M. Huang, F. W. Ye, Z. P. Sun, X. F. Chen, Opt. Express 2014, 22, 30108.

[264] K. Hao, G. Moody, F. Wu, C. K. Dass, L. Xu, C.-H. Chen, L. Sun, M.-Y. Li, L.-J.

Li, A. H. MacDonald, X. Li, Nat. Phys. 2016, 12, 677.

[265] J. R. Schaibley, H. Yu, G. Clark, P. Rivera, J. S. Ross, K. L. Seyler, W. Yao, X. Xu, Nat. Rev. Mater. 2016, 1, 16055.

[266] S. Wu, J. S. Ross, G.-B. Liu, G. Aivazian, A. Jones, Z. Fei, W. Zhu, D. Xiao, W. Yao, D. Cobden, X. Xu, Nat. Phys. 2013, 9, 149; D. Xiao, G. B. Liu, W. X. Feng, X. D. Xu, W. Yao, Phys. Rev. Lett. 2012, 108, 196802.

[267] Z. Ye, T. Cao, K. O'Brien, H. Zhu, X. Yin, Y. Wang, S. G. Louie, X. Zhang, Nature 2014, 513, 214; J. Xiao, Z. Ye, Y. Wang, H. Zhu, Y. Wang, X. Zhang, Light Sci. Appl. 2015, 4, e366.

[268] G. Bautista, M. J. Huttunen, J. Mäkitalo, J. M. Kontio, J. Simonen, M. Kauranen, Nano Lett. 2012, 12, 3207.

[269] S. Dufferwiel, T. P. Lyons, D. D. Solnyshkov, A. A. P. Trichet, WithersF,

SchwarzS, MalpuechG, J. M. Smith, K. S. Novoselov, M. S. Skolnick, D. N. Krizhanovskii, A. I. Tartakovskii, Nat. Photonics 2017, 11, 497; Y.-J. Chen, J. D. Cain, T. K. Stanev, V. P. Dravid, N. P. Stern, Nat. Photonics 2017, 11, 431; X. Liu, T. Galfsky, Z. Sun, F. Xia, E.-c. Lin, Y.-H. Lee, S. Kéna-Cohen, V. M. Menon, Nat. Photonics 2015, 9, 30; S. Dufferwiel, S. Schwarz, F. Withers, A. A. P. Trichet, F. Li, M. Sich, O. Del Pozo-Zamudio, C. Clark, A. Nalitov, D. D. Solnyshkov, G. Malpuech, K. S. Novoselov, J. M. Smith, M. S. Skolnick, D. N. Krizhanovskii, A. I. Tartakovskii, Nat. Commun. 2015, 6, 8579.

[270] G. Khitrova, H. M. Gibbs, F. Jahnke, M. Kira, S. W. Koch, Rev. Mod. Phys. 1999, 71, 1591; O. Cotleţ, S. Zeytinoğlu, M. Sigrist, E. Demler, A. Imamoğlu, Phys. Rev. B 2016, 93, 054510; T. Karzig, C.-E. Bardyn, N. H. Lindner, G. Refael, Phys. Rev. X 2015, 5, 031001 .

[271] T. Fryett, A. Zhan, A. Majumdar, arXiv 2017, 1708.05099.

[272] T. K. Fryett, A. Zhan, A. Majumdar, Opt. Lett. 2017, 42, 3586.

[273] H. Jussila, H. Yang, N. Granqvist, Z. Sun, Optica 2016, 3, 151; H. Jussila, T. Albrow-Owen, H. Yang, G. Hu, S. Aksimsek, N. Granqvist, H. Lipsanen, R. C. T. Howe, Z. Sun, T. Hasan, ACS Omega 2017, 2, 2630; Y. Chen, G. Kang, A. Shah, V. Pale, Y. Tian, Z. Sun, I. Tittonen, S. Honkanen, H. Lipsanen, Adv. Mater. Interfaces 2014, 1, 1300008. [274] A. E. Nikolaenko, N. Papasimakis, E. Atmatzakis, Z. Luo, Z. X. Shen, F. De Angelis, S. A. Boden, E. Di Fabrizio, N. I. Zheludev, Appl. Phys. Lett. 2012, 100, 181109; N. Papasimakis, Z. Luo, Z. X. Shen, F. De Angelis, E. Di Fabrizio, A. E. Nikolaenko, N. I. Zheludev, Opt. Express 2010, 18, 8353; C. In, H. Kim, B. Min, H. Choi, "Terahertz secondorder nonlinear optics in a graphene-metamaterial device: difference-frequency generation", presented at CLEO: 2014, San Jose, USA, 2014. FTh1C.6. 
[275] Y. Okawachi, K. Saha, J. S. Levy, Y. H. Wen, M. Lipson, A. L. Gaeta, Opt. Lett. 2011, 36, 3398; P. Del'Haye, A. Schliesser, O. Arcizet, T. Wilken, R. Holzwarth, T. J. Kippenberg, Nature 2007, 450, 1214.

[276] F. Wang, A. G. Rozhin, V. Scardaci, Z. Sun, F. Hennrich, I. H. White, W. I. Milne, A. C. Ferrari, Nat. Nanotechnol. 2008, 3, 738; X. Liu, D. Han, Z. Sun, C. Zeng, H. Lu, D. Mao, Y. Cui, F. Wang, Sci. Rep. 2013, 3, 2718; Z. Sun, A. G. Rozhin, F. Wang, T. Hasan, D. Popa, W. O'Neill, A. C. Ferrari, Appl. Phys. Lett. 2009, 95, 253102; Z. Sun, A. G. Rozhin, F. Wang, V. Scardaci, W. I. Milne, I. H. White, F. Hennrich, A. C. Ferrari, Appl. Phys. Lett. 2008, 93, 061114; Z. Sun, T. Hasan, F. Wang, A. G. Rozhin, I. H. White, A. C. Ferrari, Nano Res. 2010, 3, 404; E. J. R. Kelleher, J. C. Travers, Z. Sun, A. G. Rozhin, A. C. Ferrari, S. V. Popov, J. R. Taylor, Appl. Phys. Lett. 2009, 95, 111108; D. Popa, Z. Sun, T. Hasan, W. B. Cho, F. Wang, F. Torrisi, A. C. Ferrari, Appl. Phys. Lett. 2012, 101, 153107; E. J. R.

Kelleher, J. C. Travers, Z. Sun, A. C. Ferrari, K. M. Golant, S. V. Popov, J. R. Taylor, Laser Phys. Lett. 2010, 7, 790; M. Zhang, E. J. R. Kelleher, T. H. Runcorn, V. M. Mashinsky, O. I. Medvedkov, E. M. Dianov, D. Popa, S. Milana, T. Hasan, Z. Sun, F. Bonaccorso, Z. Jiang, E. Flahaut, B. H. Chapman, A. C. Ferrari, S. V. Popov, J. R. Taylor, Opt. Express 2013, 21, 23261.

[277] N. Youngblood, Y. Anugrah, R. Ma, S. J. Koester, M. Li, Nano Lett. 2014, 14, 2741. [278] J. T. Kim, K. H. Chung, C. G. Choi, Opt. Express 2013, 21, 15280.

[279] F. Torrisi, T. Hasan, W. Wu, Z. Sun, A. Lombardo, T. S. Kulmala, G.-W. Hsieh, S. Jung, F. Bonaccorso, P. J. Paul, D. Chu, A. C. Ferrari, ACS Nano 2012, 6, 2992. 